\title{
Extensive and functional overlap of the STAT6 and RXR cistromes in the active enhancer repertoire of human CD14+ monocyte derived differentiating macrophages
}

Zsolt Czimmerer $^{1,7}$, Zsuzsanna S. Nagy ${ }^{1,7}$, Gergely Nagy ${ }^{1,2}$, Attila Horvath ${ }^{1,2}$, Timea Silye-Cseh ${ }^{1}$, Agnes Kriston ${ }^{1}$, David Jonas ${ }^{1}$, Sascha Sauer ${ }^{3}$, Laszlo Steiner ${ }^{4}$ Bence Daniel ${ }^{5}$, Jean-Francois Deleuze $^{6}$ and Laszlo Nagy ${ }^{1,2,5,8}$

${ }^{1}$ Department of Biochemistry and Molecular Biology, Faculty of Medicine, University of Debrecen, Debrecen, Hungary

${ }^{2}$ Hungarian Academy of Sciences, "Lendület" Immunogenomics Research Group, University of

Debrecen, Debrecen, Hungary

${ }^{3}$ Max Delbruck Center for Molecular Medicine (BISMB and BIH), Germany

${ }^{4}$ UD-Genomed Medical Genomic Technologies Ltd., Debrecen, Hungary

${ }^{5}$ Sanford-Burnham-Prebys Medical Discovery Institute, Orlando, Florida, USA

${ }^{6}$ Centre National de Recherche en Genomique Humaine, Institute de Biologie Francois Jacob, CEA, Evry, France

${ }^{7}$ These authors contributed equally to this work.

${ }^{8}$ Corresponding author. 


\section{ABSTRACT}

Macrophages are able to differentiate into classically polarized (M1) or alternatively polarized (M2) states upon encountering pro-inflammatory cytokines such as interferon (IFN) $\gamma$ or antiinflammatory cytokines such as interleukin (IL) -4/IL-13, respectively. Moreover, macrophages are known to regulate lipid metabolism via multiple members of the nuclear hormone receptor family, including the retinoid X receptors (RXR). It has been also documented that cytokines are able to modulate macrophage responses to lipid signals but the nature of these interactions and the underlying mechanisms of these processes especially at the level of the chromatinized genome are not well understood. Previous work from our laboratory suggested that STAT6 is a facilitator of nuclear receptor mediated transcriptional activity acting at the genome level. This prompted us to investigate genome-wide DNA binding events and the development of cistromes in human CD14+ monocyte-derived macrophages upon exposure to IL-4. We determined the impact of IL-4 on the PU.1, RXR and STAT6 cistromes within the active enhancer regions marked by H3K27-acetylation using chromatin immunoprecipitation followed by deep sequencing and integrated bioinformatics analyses. We found that about $2 / 3^{\text {rd }}$ of the IL- 4 induced STAT6 peaks co-localized with RXR peaks. These STAT6/RXR co-peaks differed at least in part from the non-overlapping RXR peaks regarding the most enriched de novo transcription factor binding motifs. Interestingly, RXR-binding was not regulated at the STAT6/RXR cobound enhancers following IL-4 stimulation, but differential enhancer interactions were observed between the IL-4/STAT6 and RXR signaling pathways acting in a gene selective manner. Our results suggest that there is a novel, so far uncharacterized cistromic crosstalk between RXR and STAT6 that is likely to contribute to the formation of the active enhancer repertoire, transcriptome and differential signal-specific gene regulation of polarized macrophages.

KEYWORDS: interleukin-4, STAT6, Retinoid X Receptor, macrophage, chromatin immunoprecipitation, trancriptome, cistrome 


\section{BACKGROUND}

Macrophages are immune cells that take part in both the initiation as well as the resolution phase of inflammation, depending on the surrounding cytokine milieu. If macrophages are exposed to inflammatory cytokines such as interferon (IFN) gamma or tumor necrosis factor (TNF) alpha then classically polarized (aka M1) cells form, while anti-inflammatory cytokines such as interleukin (IL) -4 or IL-13 give rise to alternatively polarized (M2) macrophages (Gordon and Taylor, 2005,Lawrence and Natoli, 2011,Murray and Wynn, 2011).

Cytokines bind to their respective receptors that, among other signaling pathways, allow the activation of Janus tyrosine kinases (JAK), subsequently leading to the phosphorylation, dimerization and nuclear translocation of Signal Transducer and Activator of Transcription (STAT) proteins (Darnell, Kerr and Stark, 1994,Leonard, 2001). For instance, IL-4/IL-13 via JAK2 activate STAT6, a transcription factor that is known to directly bind a unique gamma activated sequence (GAS) motif with a 4 nucleotides spacer TTC(N) $)_{4} \mathrm{GAA}$ (Ehret, Reichenbach, Schindler et al., 2001,Schindler, Wu, Rothe et al., 1995) leading to the regulation of genes responsible for cell proliferation, survival and immune function (Gordon and Martinez, 2010).

Myeloid cells including dendritic cells and macrophages are also unique in their ability to sense and respond to lipids in their environment and translate these signals into transcriptional changes via certain members of the nuclear hormone receptor family including the Peroxisome Proliferator Activated Receptors (PPAR), Liver X Receptors (LXR), Vitamin D receptor (VDR) and Retinoic Acid Receptors (RAR) in heterodimers with Retinoid X Receptors (RXR) (reviewed in (Kiss, Czimmerer and Nagy, 2013)). In the absence of ligands RXR heterodimers are generally bound to DNA to act as transcriptional repressors by recruiting nuclear receptor corepressor (NCoR) or silencing mediator of retinoid and thyroid receptors (SMRT)-containing corepressor complexes (Watson, Fairall and Schwabe, 2012,McKenna, Lanz and O'Malley, 1999). On the other hand, ligand activation induces corepressor/coactivator complex exchange and consequently transcriptional activation of nuclear hormone receptor target genes (McKenna et al., 1999,Kato, Yokoyama and Fujiki, 2011). Based on the activation properties of its ligands, RXR heterodimers can be categorized as permissive or non-permissive. Permissive RXR heterodimers including PPAR/RXR or LXR/RXR- can be activated by the ligands of either partner. In contrast, non-permissive heterodimers cannot be activated by an RXR agonist but only of the dominant 
partner receptors such as VDR or RAR (Germain, Chambon, Eichele et al., 2006). These RXR heterodimers are responsible for the development of important functional characteristics including lipid metabolism and inflammatory responsiveness in different macrophage polarization states (reviewed in (Kiss et al., 2013,Varga, Czimmerer and Nagy, 2011)). In addition, liganded RXR promotes a unique gene expression program resulting in the elevated expression of pro-angiogenic genes and enhanced angiogenic activity in macrophages (Daniel, Nagy, Hah et al., 2014).

Besides the thorough investigation of the physiological consequences of cytokine and/or lipid signals in macrophages it is also important to understand the underlying epigenomic events such as transcription factor DNA binding as well as chromatin modifications as determinants of transcriptional and consequently phenotypic and functional outcomes (Lawrence and Natoli, 2011). For example, in mouse macrophages it is already known how cytokine stimuli via genomic binding events and histone posttranslational modifications induce transcriptional changes to shape macrophage activity (Lawrence and Natoli, 2011,Ostuni, Piccolo, Barozzi et al., 2013). Furthermore, important genomic and cistromic aspects, such as epigenetic enhancer signatures (Pham, Benner, Lichtinger et al., 2012), PU.1 binding (Pham, Minderjahn, Schmidl et al., 2013), transcriptome mapping upon M-CSF, GM-CSF (Beyer, Mallmann, Xue et al., 2012) or various stimuli (Xue, Schmidt, Sander et al., 2014) in human CD14+ monocyte-derived macrophages have also been reported.

It is also known that the macrophage polarization signals and nuclear hormone receptor signaling can interact with each other to modulate macrophage phenotype and functions. Among the potential RXR heterodimerization partners, PPAR $\gamma$ expression and activity is tightly regulated by different macrophage polarization stimuli (Huang, Welch, Ricote et al., 1999,Szanto, Balint, Nagy et al., 2010,Nagy, Czimmerer, Szanto et al., 2013). IL-4-activated STAT6 acts as a facilitator of PPAR $\gamma$ signaling during alternative macrophage activation (Szanto et al., 2010). In contrast, TNF $\alpha$ and IFN $\gamma$-induced classical macrophage activation is associated with repressed PPAR $\gamma$ activity in both human and murine cells (Szanto et al., 2010,Nagy et al., 2013). The importance of RARs in alternative macrophage activation pertaining to the ability of these cells to reversibly and dynamically alter their phenotypes upon the continously changing tissue environment was recently highlighted by the finding that all-trans retinoic acid is involved in the functional polarization of peritoneal macrophages via the reversible up-regulation of the GATA6 
transcription factor (Okabe and Medzhitov, 2014). It has also been described that LXR activation modulates the functional properties of human alternatively polarized macrophages (Bories, Colin, Vanhoutte et al., 2013). Little is known, however, of the molecular details of IL-4 and RXR signaling crosstalk at the epigenomic and gene expression levels to shape macrophage function. These pieces of evidence prompted us to investigate the epigenomic events that take place in these cells upon IL-4 stimulation and RXR activation. In the present work we have assessed how IL-4 modulates the human STAT6 cistrome in primary human CD14+ monocyte-derived differentiating macrophages. Moreover, we sought to understand how the IL-4 induced STAT6 cistrome and the baseline RXR cistrome might interact with one another within the context of active macrophage enhancers marked by the H3K27Ac histone modification and the macrophage lineage specific factor, PU.1. To this end, we have performed ChIP with antibodies to H3K27Ac, PU.1, RXR and STAT6 from cells left unstimulated or stimulated with IL-4. We carried out an integrated bioinformatic analysis of the data sets and established the overlapping peak sets between PU.1, RXR and STAT6. Surprisingly, we found that approximately $2 / 3^{\text {rd }}$ of the STAT6 peaks overlapped with RXR/PU.1 peaks but these were not associated with nuclear receptor (NR) half sites sufficiently similar to be found enriched with motif analysis tools, suggesting that (i) these RXR peaks likely differ from those not overlapping with STAT6, and/or (ii) RXR might be indirectly recruited to these regions. Furthermore, the STAT6/RXR/PU.1 co-peaks associated with distinct transcription factor motifs than the PU.1/RXR co-peaks, including those of RUNX and C/EBP factors, suggesting that STAT6 is recruited to genomic frequented by several factors (Siersbaek, Nielsen, John et al., 2011). Lastly, we have confirmed the differential interactions between IL-4/STAT6 and RXR signaling pathways by means of eRNA transcript quantification on a select set of target genes.

Our data revealed a novel interrelationship between the RXR and STAT6 cistromes, further strengthening the notion that lipid and cytokine signals converge in macrophages and thus our results contribute important details to the ever expanding field of immune and metabolic crossregulation.

\section{MATERIALS AND METHODS}


Monocyte isolation and differentiation, cell culture and treatments: Human monocytes were isolated from peripheral blood mononuclear cells (PBMC) of healthy volunteers. Buffy coats were obtained from the Regional Blood Bank. Monocyte separation was carried out using CD14 MicroBeads (Miltenyi Biotec) according to the manufacturer's instructions. Monocytes were cultured and differentiated to macrophages by their attachment to cell culture plate in RPMI 1640 supplemented with $10 \%$ FBS, $2 \mathrm{mM}$ glutamine, penicillin and streptomicyn for the indicated time. (Liu, Shi, Huang et al., 2008,Perlman, Pagliari, Georganas et al., 1999,Pagliari, Perlman, Liu et al., 2000,Liu, Perlman, Pagliari et al., 2001) Cells were stimulated as indicated with IL-4 (20 ng/ml) for the time specified within the figure legends. LG268 was used at $100 \mathrm{nM}$ final concentrations, respectively for the time indicated in the figure legends. DMSO-Ethanol served as vehicle control $(0.01 \%)$.

Chromatin immunoprecipitation and ChIP library preparation: Human CD14+ monocytederived differentiating macrophages at 24 hours following isolation were treated with IL-4 (20 $\mathrm{ng} / \mathrm{ml}, 30 \mathrm{~min}$ ) as indicated then harvested by scraping in medium followed by centrifugation at 4C for $10 \mathrm{~min}$ at $1000 \mathrm{~g}$ then cross-linked using DSG and formaldehyde-based double crosslinking method and processed for ChIP. Chromatin was immunoprecipitated with antibodies against PU.1 (sc-352), STAT6 (sc-981), RXR (sc-774) and H3K27Ac (ab4729). Library preparation and analysis were performed as previously described (Nagy, Daniel, Jonas et al., 2013) with minor modifications. $1 \mathrm{ng}$ (STAT6, PU.1, RXR) and 5 ng (H3K27Ac) DNA amounts were used for the library preparation.

ChIP-seq analysis: The primary analysis of raw sequence reads has been carried out using our ChIP-seq_analyze command line pipeline (Barta, 2011). Alignment to the hg19 genome assembly was made by the Burrows-Wheeler Alignment (BWA) tool (Li and Durbin, 2009). Genome coverage (bedgraph) files were made by makeUCSCfile.pl (Homer) (Heinz, Benner, Spann et al., 2010) and used for visualization with IGV 2.3 (Thorvaldsdottir, Robinson and Mesirov, 2013). EBI PeakSplitter (Bertone Group Software) was used for obtaining regions occupied by H3K27ac modified histones as described in (Nagy et al., 2013) with minor modifications. The thus predicted regions of the vehicle and IL-4 treated samples were extended by $0.5 \mathrm{~kb}$ in both directions and then merged (BEDtools) (Quinlan and Hall, 2010). Transcription factor ChIP-seq 
peaks were predicted by Homer findPeaks $(\mathrm{p}<0.0001)$, and false enrichments according to our homemade blacklist and those do not exceed 3 RPKM read density were eliminated. Consensus peak sets for PU.1, RXR and STAT6 were determined by DiffBind: Two biological replicates for both experimental condition (control and IL-4 treatment) were used; and those peaks that could be predicted from at least any two of four samples were merged and regarded as consensus peaks (Ross-Innes, Stark, Teschendorff et al., 2012). For further analyses peaks overlapping with the H3K27ac modified regions were used. Peak set overlaps were defined by intersectBed (BEDTools) and visualized by VennMaster-0.37.5 (Kestler, Muller, Gress et al., 2005). Annotations and read distribution histograms centered to peaks were made by annotatePeaks.pl (Homer). Read distribution heat maps were created by Java TreeView in $\log 2$ scale from the output of annotatePeaks.pl. Summit +/-100 bp of all or the top 1000 peaks (based on the highest Homer peak score of the merges) was used for the prediction of motif enrichments by findMotifsGenome.pl (Homer). Repeat masking and 10, 12, 14 and 16 search length were set. Target percent refers to the ratio of the peaks having the given motif, and background ( $\mathrm{Bg})$ percent shows the motif enrichment of a large random sequence collection with 200-base lengths. P-values were calculated based on the enrichments of these two sets of regions. The used command line scripts are available upon request.

RNA-Seq: Indexed cDNA libraries were prepared from $1 \mu \mathrm{g}$ of total RNA following the TruSeq $^{\text {TM }}$ RNA Sample Preparation Kit (Illumina, Inc.) protocol. Briefly, the mRNA Poly-A was selected on oligo (dT) magnetic beads and fragmented by divalent cations at $94^{\circ} \mathrm{C}$ for $5 \mathrm{~min}$. Reverse transcription was performed using SuperScript II reverse transcriptase (Life Technologies, Inc.) and random primers. After second strand cDNA synthesis, fragments were end repaired, A-tailed and ligated with indexed adapters. cDNA libraries were purified and size selected after PCR with AMPure XP beads (Beckman Coulter, Inc, Fullerton, C.A USA). The average size of inserts was $132 \pm 17 \mathrm{bp}$. The $100 \mathrm{bp}$ paired-end sequencing of the cDNA libraries was performed on pools of 4 samples in equal ratio in a single lane of the Illumina Hiseq 2500 sequencer. Quality criteria were checked during the run $(\mathrm{Q} 30$, base distribution, error rate, etc.). Raw images were processed for base calling and fastq files went through a quality check pipeline (duplication rate, coverage, number of genes/transcripts, etc.). The TopHat-Cufflinks-Cuffdiff toolkit trio (Trapnell, Hendrickson, Sauvageau et al., 2013) was used for mapping spliced reads, 
making transcript assemblies. Downstream data analyses were performed in R (Team, 2013). Bar plots and clustering were created using R packages ggplot2 and stats, respectively (Kolde, 2013).

Real-Time Quantitative PCR for enhancer RNA and mRNA detection (qPCR): RNA was isolated with Trizol reagent (Ambion). RNA was reverse transcribed with High-Capacity cDNA Reverse Transcription Kit (Applied Biosystems) according to manufacturer's protocol. Transcript quantification was performed by qPCR reactions using SYBR green master mix (BioRad). Transcript levels were normalized to GAPDH. Primers are available upon request.

\section{RESULTS AND DISCUSSION}

\section{Investigation of IL-4-modulated gene expression signature in primary human monocyte- derived differentiating macrophages.}

In order to investigate the potential interaction between IL-4/STAT6 and RXR signaling in human differentiating macrophages (diffMQ), we induced the differentiation of human peripheral blood-derived CD14+ monocytes to macrophages by their attachment to the cell culture plate.

First, we validated the experimental cell model as depicted in Figure 1A by testing IL-4 responsiveness using RNA-seq methodology to confirm that the cells were able to differentiate towards the M2 phenotype (Figure 1B). We observed that following IL-4 treatment 826 genes showed a minimum of 1.5 fold differences including the previously described IL-4-induced alternative macrophage activation markers MRC1, SOCS1, MAOA, TGM2, SLA, CD180 and ENPP2 in both donors (Figure 2A and B) (Gordon and Martinez, 2010,Czimmerer, Varga, Poliska et al., 2012,Martinez, Helming, Milde et al., 2013). Next, we aimed to determine, which biological processes were affected in diffMQ cells by IL-4. Using DAVID Bioinformatics Database (Huang da, Sherman and Lempicki, 2009), we found that the IL-4-regulated genes were associated with inflammation and lymphocyte chemotaxis-linked biological processes (Figure 2C). Next, we determined the expression levels of RXR isoforms and their potential heterodimerization partners in unstimulated and IL-4 stimulated diffMQ cells using our RNA-seq 
data set. In agreement with the previously published studies (Roszer, Menendez-Gutierrez, Cedenilla et al., 2013), two out of three RXR isoforms such as RXR $\alpha$ and RXR $\beta$ were expressed $(R P K M \geq 1$ at least one diffMQ sample) in our experimental cell model (Figure 2D). In addition, 9 potential RXR heterodimerization partners were also expressed at least one human donor-derived unstimulated diffMQ cells including the most abundantly expressed LXR $\alpha$, LXR $\beta$, PPAR $\delta$, VDR and RAR $\alpha$ (Figure 2D). Finally, the M2-type polarization-mediated regulation of three nuclear receptors was observed in both human donors-derived diffMQ cells including the IL-4-induced PPAR $\gamma$ and VDR as well as the repressed LXR $\alpha$ (Figure 2D).

These data demonstrate that the ex vivo-derived diffMQ cells were likely polarized toward the M2 phenotype following IL-4 treatment and expressed two RXR isoforms and several of their heterodimerization partners.

\section{Mapping PU.1, RXR and STAT6 occupied enhancer regions in human CD14+ monocyte- derived differentiating macrophages.}

In order to gain insight into how IL-4 driven cistromic changes relate to macrophage and/or lipid mediated genomic events in diffMQ cells, first we differentiated macrophages from human peripheral blood-dervied CD14+ monocytes and performed ChIP-seq studies, as depicted in Figure $1 \mathrm{~A}$ and $\mathrm{B}$.

In this set of experiments we identified chromatin regions that harbor the active enhancer mark, histone 3 acetylated at lysine 27 (H3K27Ac) (Creyghton, Cheng, Welstead et al., 2010) as well as genomic binding regions of the myeloid lineage-specific macrophage pioneer factor PU.1 (Simon, Olson, Scott et al., 1996,Friedman, 2007, Ghisletti, Barozzi, Mietton et al., 2010), the IL4-driven transcription factor STAT6 and the lipid mediated transcription factor RXR. Next, we comparatively analyzed the genomic data sets using integrated bioinformatic tools as described in the Materials and Methods. As shown in Figure 3A, 20,046 PU.1 (75\% of 26,531 total), 12,799 (90\% of 14,125) RXR and 1,417 (93\% of 1,573) STAT6 peaks were found positive for the H3K27Ac active enhancer mark. The Venn diagram also represents the overlap among the peaks occupied by these transcription factors (TFs): approximately $75 \%$ of RXR peaks and $80 \%$ of STAT6 peaks fell within regions positive for PU.1 ( 7,600 and $\sim 1,200$, respectively). Intriguingly, about $60 \%$ of STAT6 peaks overlapped with RXR peaks $(\sim 1,000)$, a phenomenon 
which, to our knowledge, has not yet been reported in the scientific domain for these two factors (Figure 3A and B). On the other hand, PU.1 binding sites in relation to those of PPARG/RXR in Thp-1 cells (Pott, Kamrani, Bourque et al., 2012) or PPARG in mouse thioglycolate-elicited macrophages (Lefterova, Steger, Zhuo et al., 2010) were also found to overlap approximately at $60 \%$, which is in good correlation with our results.

Next, we examined the IL-4-modulated binding of RXR, PU.1 and STAT6 transcription factors at the H3K27Ac and PU.1 double-positive genomic regions (Figure 3B). First, it should be emphasized that according to the current dogma STATs only become phosphorylated, dimerize and translocate to the nucleus to bind DNA upon cytokine stimulation (Darnell et al., 1994,Leonard, 2001). In line with this, STAT6 peaks (blue) only occur in the IL-4 treated samples but not in unstimulated cells as shown in Figure 3B. PU.1 (red) is known to constitutively occupy enhancers in mouse macrophages (Ghisletti et al., 2010), which is in agreement with our results in these cells. So far, genomic RXR binding sites were mapped during mouse adipogenesis (Nielsen, Pedersen, Hagenbeek et al., 2008,Hamza, Pott, Vega et al., 2009), in mouse osteoblast cells (Meyer, Goetsch and Pike, 2010) and in the human monocytic cell line, Thp-1 (Pott et al., 2012). In addition, a previous study from our laboratory described the constitutive baseline landscape of RXR binding in unstimulated mouse bone marrow derived macrophages (Daniel et al., 2014), strengthening our findings. However, the effect of a short term treatment with IL-4 (30 min) has not yet been published in any human macrophage system; hence the baseline constitutive in vivo kinetics of RXR DNA binding (green) observed in the present work could not be supported by independent earlier results.

Finally, we investigated the IL-4-mediated effects on H3K27Ac enrichment at the RXR, STAT6 and RXR+STAT6 co-bound H3K27Ac and PU.1 double-positive genomic regions in IL-4 stimulated and unstimulated diffMQ cells (Figure 3C). IL-4-induced H3K27Ac enrichment was observed at both STAT6 and RXR/STAT6 co-bound genomic regions, however, both basal and IL-4-enhanced H3K27 acetylation were lower at the STAT6 bound genomic regions compared to the RXR/STAT6 co-bound genomic sites (Figure 3C). These findings suggest the IL-4-dependent activation of both STAT6 and RXR+STAT6 co-bound regulatory elements in diffMQ cells.

\section{Genomic distribution and motif analysis of PU.1, RXR and STAT6 occupied regions.}


As the next step, we classified the location of the peaks relative to coding genes. We established the following categories: promoter-TSS, 5' UTR, exon, intron, 3' UTR, TTS, non-coding and intergenic (Figure 4A). We found that PU.1 peaks localized to promoter-TSS, intronic and intergenic regions at 13, 44 and 37\%, respectively; while RXR peaks localized to promoter-TSS, intronic and intergenic regions at 24, 35 and 34\%, respectively. On the other hand, STAT6 peaks were distributed between introns and intergenic regions at 42 and 49\%, respectively. In light of the significant overlap between RXR and STAT6 peaks, it was not surprising that the RXR/STAT6 co-peaks were at a similar extent split between these two major categories (41 and $49 \%$ ). The genomic localization of STAT6 examined in mouse or human Th2 cells was 59\% or $51 \%$ intergenic, respectively; in good agreement with our findings (Elo, Jarvenpaa, Tuomela et al., 2010,Wei, Vahedi, Sun et al., 2010).

Next, we analyzed the consensus motifs under the ChIP-Seq peaks identified using the HOMER toolkit ((Hamza et al., 2009), see Matherial and Methods) (Figure 4B). First, the top 2000 peaks of PU.1 and RXR were analyzed. The most significantly enriched motifs for PU.1 were PU.1 and C/EBP, while nuclear receptor (NR) half sites, PU.1, C/EBP followed by DR4, AP-1, DR1 and REL were found for RXR. The latter list was compiled using de novo motif search. Then, the overlapping peaks between PU.1 and RXR were analyzed for the top 1000 PU.1 peaks, which harbored enriched PU.1 motifs, and the same held true for the top RXR peaks within this peak set, followed by the C/EBP, DR4, AP-1 and NR half sites. The appearance of DR4 is intriguing and may suggest that in this type of cell RXR might act by heterodimerizing with partner(s) preferentially bound to this motif, such as LXR. In agreement with this finding, LXR $\alpha$ and $\beta$ were the most abundantly expressed RXR heterodimerization partners in differentiating human macrophages (Figure 2D). PU.1 and STAT6 shared approximately 300 peaks, in which category the PU.1 motif was also the most significantly enriched one, followed by the STAT6 consensus motif then the CCAAT/enhancer-binding protein (C/EBP) and Activator Protein (AP) -1 motifs. The proximity of AP-1 and C/EBP sites to PU.1 sites was already demonstrated in mouse macrophages (Heinz et al., 2010,Lefterova et al., 2010). Surprisingly, when testing the enhancers marked by all three factors, even the forced motif search failed to reveal potential binding sites for RXR, suggesting that (i) RXR recruitment to these regions could be indirectly mediated (i.e. 
independently from direct RXR DNA binding) or (ii) RXR might recognize non-canonical binding sites.

\section{Determination of the interactions between IL-4/STAT6 and RXR signaling pathways in differentiating macrophages.}

In order to investigate whether the lipid sensing RXRs can influence IL-4/STAT6 signaling pathway-mediated transcriptional changes in diffMQ cells, we annotated the RXR and STAT6 peaks to IL-4-regulated genes. We found that $26 \%$ of STAT6 and $29 \%$ of RXR+STAT6 co-peaks were observed within $\pm 100 \mathrm{~Kb}$ distance from TSSs of IL-4-regulated genes (Figure 5A). In addition, $16 \%$ of RXR peaks were also associated with IL-4 responsive genes (Figure 5A). Interestingly, the distribution of the annotated peaks relative to TSS is fairly symmetrical in all the three peak sets (Figure 5B). Next, we decided to determine how the ligand-activated RXR signaling pathway can modulate IL-4-induced transcriptional events at the gene expression level in diffMQ cells. We selected 7 IL-4-activated and RXR/STAT6 co-bound enhancer-associated genes including well characterized alternative macrophage activation markers and immunemodulator genes (Gordon and Martinez, 2010,Czimmerer et al., 2012, Martinez et al., 2013,Colotta, Saccani, Giri et al., 1996,Scotton, Martinez, Smelt et al., 2005), and analyzed the IL-4-dependent induction of their expression at the mRNA level in the presence or absence of the synthetic RXR ligand LG268 using RT-QPCR (Figure 5C). Interestingly, 3 out of the 7 selected genes including CCL26, IL1RN and FCER2 were not regulated by LG268 alone, but their IL-4induced expression levels were enhanced in the presence LG268 compared to the IL-4 and vehicle-treated diffMQ cells suggesting the potentiating role of ligand-activated RXR in this process (Figure 6A). Our previous findings documented the existence of an extensive crosstalk between the IL-4-activated STAT6 and the synthetic PPAR $\gamma$ ligand rosiglitazone-activated permissive RXR/PPAR $\gamma$ heterodimers in human and mouse alternatively polarized macrophages (Szanto et al., 2010). However, 2 out of 3 LG268 facilitated genes such as IL1RN and CCL26 expression were not regulated in unstimulated and IL-4-stimulated human monocyte-derived differentiating macrophages by rosiglitazone (Szanto et al., 2010). These findings suggest that the liganded RXR-mediated potentiation of IL1RN and CCL26 expression was a permissive $\mathrm{RXR} / \mathrm{PPAR} \gamma$ heterodimer-independent process in IL-4 treated diffMQ cells. In addition, IL-4- 
activation of STAT6 and the rosiglitazone-activation of PPAR $\gamma$ transcription factors had an additive effect on the gene expression level of FCER2 in human differentiating macrophages (Szanto et al., 2010). Interestingly, we found that ligand activation of RXR could enhance FCER2 expression in the presence of IL-4 suggesting, that (i) FCER2 expression is regulated by non-canonical RXR/PPAR $\gamma$ permissive heterodimer or (ii) this process might be PPAR $\gamma$ independent. The well established alternative macrophage polarization marker and nuclear receptor target gene TGM2 was induced in both IL-4 and LG268-stimulated cells and its expression level was synergistically induced in co-treated cells (Figure 6A) (Daniel et al., 2014,Martinez et al., 2013,Rebe, Raveneau, Chevriaux et al., 2009,Nagy, Saydak, Shipley et al., 1996). Similar synergistic interaction was observed between IL-4/STAT6 and ligand-activated PPAR $\gamma$ signaling pathways in the case of several PPAR $\gamma$ target genes such as FABP4 and PDK4 in human differentiating macrophages (Szanto et al., 2010). However, TGM2 was not induced by rosiglitazone suggesting, that the synergistic crosstalk between IL-4/STAT6 and liganded RXR signaling pathways was not impacted by liganding PPAR (Szanto et al., 2010). It is not clear though if the presence of the receptor (PPAR $\gamma$ ) was required. It has been reported that TGM2 is a direct target gene of RXR/RAR signaling pathway in human and mouse macrophages (Rebe et al., 2009,Nagy et al., 1996). Although, RARs are known as non-permissive heterodimerization partners of RXRs, but we observed the induction of TGM2 expression and activation of TGM2associated enhancers in mouse macrophages by both RXR and RAR-specific synthetic agonists (Daniel et al., 2014). These results raised the possibility that non-canonical permissive RXR/RAR heterodimers can regulate TGM2 expression. However the RXR/RAR hetrodimer's participation in the IL-4 and LG268-medited synergistic induction of TGM2 expression remains to be determined. Finally, we found that the basal and IL-4-induced expression of 3 selected genes such as CLEC4G, CD209 and CISH were not regulated by LG268 suggesting (i) the existence of non-permissive heterodimer-dependent regulatory mechanisms or (ii) their insensitivity for nuclear receptor ligands (Figure 6A).

Next, we wanted to investigate whether the crosstalk between the ligand-activated RXR and IL4/STAT6 signaling pathways is also observed at the selected IL-4 responsive genes-associated RXR/STAT6 co-bound enhancers. It has been recently described that enhancer RNA (eRNA) expression is a suitable marker of enhancer activity (Natoli and Andrau, 2012,Simandi, Horvath, Nagy et al., 2016,Daniel, Nagy and Nagy, 2014). Therefore, we measured the IL-4 and/or 
LG268-modulated eRNA expression at 6 selected enhancers by RT-QPCR. We detected the IL-4induced activation of eRNA expression at all selected enhancers, but the RXR ligand-induced modulation of IL-4 responsiveness was distinct and only partially overlapped if compared to the mRNA expression (Figure 6B). The potentiating role of LG268 on IL-4 responsiveness was detected at CCL26_-6,5Kb and IL1RN_-13Kb enhancers similarly to mRNA expression (Figure 6B). In addition, the synergistic action of LG268 and IL-4 was also observed at the TGM2_3,3Kb enhancer (Figure 6B). The IL-4 and LG268-dependent co-regulation of eRNA expression at the above examined RXR-STAT6 co-peak-bound enhancers raises the possibility of an extensive interactions between RXR and STAT6 transcription factors including liganded RXR-facilitated STAT6 binding or co-activator recruitment. However, the investigation of the molecular bases of synergistic and potentiating interactions between RXR and STAT6 requires further analysis. Interestingly, unlike in the case of FCER2 mRNA expression, LG268 did not enhance the IL-4induced eRNA expression at FCER2_-2,3Kb enhancer (Figure 6B). However, FCER2 gene was associated with other RXR-bound regulatory region (Figure 5C) suggesting that the complex interaction of several RXR and STAT6 bound enhancers likely contribute to the synthetic RXR ligand-dependent potentiation of IL-4-induced FCER2 expression. Finally, as we expected, CD209_-13Kb and CISH_+16Kb enhancer activities were not influenced by ligand-activated RXR (Figure 6B).

Taken together, these results suggest that the lipid sensing RXR and IL-4/STAT6 signaling pathways can interact displaying different modalities (synergistic, potentiating or liganded RXRindependent) in an enhancer and gene specific manner with each other providing combinatorial gene expression and modulating phenotypic properties of human macrophages (Figure 7). These observations are integral part of our laboratory's systematic efforts, to better understand the roles of RXRs in the genome-wide organization of nuclear receptor cistromes and the interactions between nuclear receptors and other signal-dependent transcription factors in different cell types. (Daniel et al., 2014, Simandi, Horvath, Wright et al., 2016, Simandi et al., 2017) Nevertheless, the detailed characterization of the interactions between IL-4/STAT6 and liganded RXR signaling pathways requires further experimental analysis applying RXR antagonist or deleting $\operatorname{RXR} \alpha$ and $\beta$ isoforms in human macrophages.

\section{CONCLUSIONS}


Taken together, herein we provided and described several novel functional interactions deduced from extensive mapping of both cistromic as well as transcriptomic events induced by IL-4 alone or in combination with LG268 in primary human macrophage-like cells. These include (i) the cistromic maps of PU.1, RXR and STAT6 in primary macrophage like cells, (ii) the cistromic overlap between STAT6 and RXR, (iii) the lack of NR motif within these co-peaks, (iv) the gene and/or enhancer-specific synergistic and potentiating cross-talk between ligand-acitvated RXR and IL-4/STAT6 signaling pathways. These findings suggest that there is an extensive interplay between ligand-activated RXR and IL-4/STAT6 signaling pathways at both the cistromic and transcriptomic levels.

More generally, our results further reinforce the notion that it is likely that the lipid microenvironment is insufficient to induce macrophage polarization alone. However it is able to modulate IL-4 responsiveness of primary human macrophage-like cells enhancing and potentially reinforcing the alternative macrophage polarization cellular phenotype. Therefore, RXR signaling may be a potential therapeutic target in alternative macrophage activation-associated diseases including parasites infections and tumor development.

\section{ABBREVIATIONS}

JAK, Janus tyrosine kinases; STAT, Signal Transducer and Activator of Transcription; IL-4, Interleukin 4; TNFa, tumor necrosis factor alpha; IFN $\gamma$, interferon gamma; PPAR, Peroxisome Proliferator Activated Receptors; LXR, Liver X Receptors; VDR, Vitamin D receptor; RAR, Retinoic Acid Receptors; RXR, in heterodimers with Retinoid X Receptors; diffMQ, human monocyte-derived differentiating macrophage; NCoR, nuclear receptor corepressor; SMRT, silencing mediator of retinoid and thyroid receptors

\section{COMPETING INTERESTS}

No competing interests are declared.

\section{AUTHORS' CONTRIBUTIONS}


ZC and ZSN designed and performed experiments, analyzed the data and wrote the paper, TSCS and AK performed experiments, SS, JF-D, DJ, AH, GN and LS analyzed data, and LN designed experiments, wrote the paper, obtained funding and directed the research.

\section{ACKNOWLEDGEMENTS}

This research was supported by the European Union and the State of Hungary, co-financed by the European Social Fund in the framework of TÁMOP 4.2.4. A/2-11-1-2012-0001 'National Excellence Program'. Work in the Nagy laboratory is supported by a grant from the Hungarian Scientific Research Fund (OTKA K100196), and TÁMOP422_2012_0023 VÉD-ELEM implemented through the New Hungary Development Plan co-financed by the European Social Fund and the European Regional Development Fund. Z.S.N. was the recipient of the János Bolyai Research Felowship from the Hungarian Academy of Sciences (2010-2013) and was supported by the NKTH-OTKA-EU 7KP (Marie Curie actions) Reintegration Grant. The authors would like to thank the members of the Nuclear Hormone Receptor laboratory for their help and comments. The authors would like to acknowledge the excellent skillful technical assistance of Drs. Szilárd Póliska and Tibor Gyuris at the Clinical Genomics Center at the University of Debrecen for performing the certain parts of the ChIP sequencing. ChIP library preparation and bioinformatic analysis was performed at the Center of Clinical Genomics and Personalized Medicine of the University of Debrecen. Sequencing was performed at the Centre National de Genotypage (CNG) Paris, funded by the ESGI (European Sequencing and Genotyping Infrastructure) Consortia as part of the ADIPOMACTX transnational access program.

ACCESSION NUMBERS Sequencing data have been submitted to GEO database under accession: GSE100889. 


\section{FIGURE LEGENDS}

Figure 1. Schematic representation of the differentiation protocol and the experimental design. (A) The differentiation protocol of human CD14+ monocyte derived diffMQ cells as described in the Materials and Methods section is shown. (B) Schematic representation of the experimental setup for the cistromic and transcriptomic analyses of diffMQ cells.

Figure 2. Identification of novel IL-4 responsive genes in diffMQ cells. (A) RNA expression of known human alternative macrophage activation markers in control and IL-4-stimulated diffMQ cells. diffMQ cells were treated with IL-4 for 4 hours. The selected markers were visualized in the Integrated Genome Viewer (IGV). (B) Heat map showing fold changes of IL-4 responsive transcripts in unstimulated (ctrl) and IL-4-stimulated (IL-4) diffMQ cells. diffMQ cells were treated with IL-4 for 4 hours. (C) Top 10 most significantly enriched biological processes within IL-4-regulated genes identified by DAVID Bioinformatics Database. (D) Heat map showing RPKM values of the expressed RXR isoforms and their potential hetrodimerization partners in unstimulated (ctrl) and IL-4-stimulated (IL-4) diffMQ cells. diffMQ cells were treated with IL-4 for 4 hours.

Figure 3. Genome-wide mapping of H3K27Acetylated PU.1, RXR and STAT6 occupied regions in diffMQ cells. (A) Venn diagram represents overlapping peaks among H3K27Acetylated PU.1, RXR and STAT6 occupied regions. Table contains total number of peaks occupied by each transcription factor overall (consensus) or in an active (H3K27Ac positive) state. (B) Read distribution plot of ChIP-seq intensities against H3K27Ac, PU.1, RXR and STAT6 around the detected PU.1 peaks in $4 \mathrm{~Kb}$ window in both control and IL-4-stimulated diffMQ cells. (C) Box plot representation of H3K27Ac marks at the surrounding genomic regions of RXR, STAT6 and RXR+STAT6 peaks in in control or IL-4-stimulated diffMQ cells.

Figure 4. Characterization of RXR, STAT6 and RXR-STAT6 peak sets. (A) Genomic distribution of RXR, STAT6 and RXR+STAT6 peaks relative to gene coding regions expressed as \% of total PU.1 positive peak numbers. (B) De novo motif enrichment under RXR, STAT6 and RXR+STAT6 peaks from ChIP-seq data using HOMER. "Target \%" refers to the ratio of the peaks having the given motif, and "Bg \%" refers to the ratio of a random background.

Figure 5. Identification of IL-4 responsive gene associated RXR, STAT6 and RXR+STAT6 peaks. (A) Annotation of RXR, STAT6 and RXR+STAT6 peaks of IL-4 responsive genes within 
$\pm 100 \mathrm{~Kb}$ distance relative to TSS. (B) Distribution of RXR, STAT6 and RXR+STAT6 peaks relative to TSSs of the IL-4-regulated genes. (C) H3K27Ac, PU.1, STAT6 and RXR ChIP-Seq as well as RNA-seq signals at select genomic regions in IL-4-stimulated or unstimulated diffMQ cells. ChIP-seq and RNA-seq signals for the indicated factors, as well as post-translational histone modification and mRNA expression are visualized by the Integrative Genomics Viewer.

Figure 6. Characterization of potential interactions between IL-4/STAT6 and RXR signaling pathways at the gene and enhancer levels. (A) qRT-PCR measurement of the expression of select mRNAs in vehicle (veh), IL-4 (IL-4/veh), RXR ligand LG268 (LG268) or costimulated (IL-4/LG268) monocyte-derived diffMQ cells from two independent human donors (D1 and D2). diffMQ cells were treated with IL-4 and LG268 for 4 hours. (B) qRT-PCR measurement of eRNA expression at the enhancers of the select IL-4-responsive genes in control (veh), IL-4 (IL-4/veh), RXR ligand LG268 (LG268) or costimulated (IL-4/LG268) diffMQ cells. diffMQ cells were treated with IL-4 and LG268 for 30, 60 minutes and 4 hours.

\section{Figure 7. Schematic representation of the potential interactions between IL-4/STAT6 and ligand-activated RXR signaling pathways in diffMQ cells.}

\section{REFERENCES}

[1] Gordon, S. and Taylor, P.R., 2005. Monocyte and macrophage heterogeneity, Nat Rev Immunol. 5, 953-64.

[2] Lawrence, T. and Natoli, G., 2011. Transcriptional regulation of macrophage polarization: enabling diversity with identity, Nat Rev Immunol. 11, 750-61.

[3] Murray, P.J. and Wynn, T.A., 2011. Protective and pathogenic functions of macrophage subsets, Nat Rev Immunol. 11, 723-37.

[4] Darnell, J.E., Jr., Kerr, I.M. and Stark, G.R., 1994. Jak-STAT pathways and transcriptional activation in response to IFNs and other extracellular signaling proteins, Science. 264, 1415-21.

[5] Leonard, W.J., 2001. Role of Jak kinases and STATs in cytokine signal transduction, Int J Hematol. 73, 271-7.

[6] Ehret, G.B., Reichenbach, P., Schindler, U., Horvath, C.M., Fritz, S., Nabholz, M. and Bucher, P., 2001. DNA binding specificity of different STAT proteins. Comparison of in vitro specificity with natural target sites, J Biol Chem. 276, 6675-88.

[7] Schindler, U., Wu, P., Rothe, M., Brasseur, M. and McKnight, S.L., 1995. Components of a Stat recognition code: evidence for two layers of molecular selectivity, Immunity. 2, 689-97.

[8] Gordon, S. and Martinez, F.O., 2010. Alternative activation of macrophages: mechanism and functions, Immunity. 32, 593-604.

[9] Kiss, M., Czimmerer, Z. and Nagy, L., 2013. The role of lipid-activated nuclear receptors in shaping macrophage and dendritic cell function: From physiology to pathology, J Allergy Clin Immunol. 132, 264-86. 
[10] Watson, P.J., Fairall, L. and Schwabe, J.W., 2012. Nuclear hormone receptor co-repressors: structure and function, Mol Cell Endocrinol. 348, 440-9.

[11] McKenna, N.J., Lanz, R.B. and O'Malley, B.W., 1999. Nuclear receptor coregulators: cellular and molecular biology, Endocr Rev. 20, 321-44.

[12] Kato, S., Yokoyama, A. and Fujiki, R., 2011. Nuclear receptor coregulators merge transcriptional coregulation with epigenetic regulation, Trends Biochem Sci. 36, 272-81.

[13] Germain, P., Chambon, P., Eichele, G., Evans, R.M., Lazar, M.A., Leid, M., De Lera, A.R., Lotan, R., Mangelsdorf, D.J. and Gronemeyer, H., 2006. International Union of Pharmacology. LXIII. Retinoid $X$ receptors, Pharmacol Rev. 58, 760-72.

[14] Varga, T., Czimmerer, Z. and Nagy, L., 2011. PPARs are a unique set of fatty acid regulated transcription factors controlling both lipid metabolism and inflammation, Biochim Biophys Acta. $1812,1007-22$.

[15] Daniel, B., Nagy, G., Hah, N., Horvath, A., Czimmerer, Z., Poliska, S., Gyuris, T., Keirsse, J., Gysemans, C., Van Ginderachter, J.A., Balint, B.L., Evans, R.M., Barta, E. and Nagy, L., 2014. The active enhancer network operated by liganded RXR supports angiogenic activity in macrophages, Genes Dev. 28, 1562-77.

[16] Ostuni, R., Piccolo, V., Barozzi, I., Polletti, S., Termanini, A., Bonifacio, S., Curina, A., Prosperini, E., Ghisletti, S. and Natoli, G., 2013. Latent enhancers activated by stimulation in differentiated cells, Cell. 152, 157-71.

[17] Pham, T.H., Benner, C., Lichtinger, M., Schwarzfischer, L., Hu, Y., Andreesen, R., Chen, W. and Rehli, M., 2012. Dynamic epigenetic enhancer signatures reveal key transcription factors associated with monocytic differentiation states, Blood. 119, e161-71.

[18] Pham, T.H., Minderjahn, J., Schmidl, C., Hoffmeister, H., Schmidhofer, S., Chen, W., Langst, G., Benner, C. and Rehli, M., 2013. Mechanisms of in vivo binding site selection of the hematopoietic master transcription factor PU.1, Nucleic Acids Res. 41, 6391-402.

[19] Beyer, M., Mallmann, M.R., Xue, J., Staratschek-Jox, A., Vorholt, D., Krebs, W., Sommer, D., Sander, J., Mertens, C., Nino-Castro, A., Schmidt, S.V. and Schultze, J.L., 2012. High-resolution transcriptome of human macrophages, PLoS One. 7, e45466.

[20] Xue, J., Schmidt, S.V., Sander, J., Draffehn, A., Krebs, W., Quester, I., De Nardo, D., Gohel, T.D., Emde, M., Schmidleithner, L., Ganesan, H., Nino-Castro, A., Mallmann, M.R., Labzin, L., Theis, H., Kraut, M., Beyer, M., Latz, E., Freeman, T.C., Ulas, T. and Schultze, J.L., 2014. Transcriptomebased network analysis reveals a spectrum model of human macrophage activation, Immunity. 40, 274-88.

[21] Huang, J.T., Welch, J.S., Ricote, M., Binder, C.J., Willson, T.M., Kelly, C., Witztum, J.L., Funk, C.D., Conrad, D. and Glass, C.K., 1999. Interleukin-4-dependent production of PPAR-gamma ligands in macrophages by 12/15-lipoxygenase, Nature. 400, 378-82.

[22] Szanto, A., Balint, B.L., Nagy, Z.S., Barta, E., Dezso, B., Pap, A., Szeles, L., Poliska, S., Oros, M., Evans, R.M., Barak, Y., Schwabe, J. and Nagy, L., 2010. STAT6 transcription factor is a facilitator of the nuclear receptor PPARgamma-regulated gene expression in macrophages and dendritic cells, Immunity. 33, 699-712.

[23] Nagy, Z.S., Czimmerer, Z., Szanto, A. and Nagy, L., 2013. Pro-inflammatory cytokines negatively regulate PPARgamma mediated gene expression in both human and murine macrophages via multiple mechanisms, Immunobiology. 218, 1336-44.

[24] Okabe, Y. and Medzhitov, R., 2014. Tissue-specific signals control reversible program of localization and functional polarization of macrophages, Cell. 157, 832-44.

[25] Bories, G., Colin, S., Vanhoutte, J., Derudas, B., Copin, C., Fanchon, M., Daoudi, M., Belloy, L., Haulon, S., Zawadzki, C., Jude, B., Staels, B. and Chinetti-Gbaguidi, G., 2013. Liver X receptor activation stimulates iron export in human alternative macrophages, Circ Res. 113, 1196-205. 
[26] Siersbaek, R., Nielsen, R., John, S., Sung, M.H., Baek, S., Loft, A., Hager, G.L. and Mandrup, S., 2011. Extensive chromatin remodelling and establishment of transcription factor 'hotspots' during early adipogenesis, EMBO J. 30, 1459-72.

[27] Liu, H., Shi, B., Huang, C.C., Eksarko, P. and Pope, R.M., 2008. Transcriptional diversity during monocyte to macrophage differentiation, Immunol Lett. 117, 70-80.

[28] Perlman, H., Pagliari, L.J., Georganas, C., Mano, T., Walsh, K. and Pope, R.M., 1999. FLICEinhibitory protein expression during macrophage differentiation confers resistance to fasmediated apoptosis, J Exp Med. 190, 1679-88.

[29] Pagliari, L.J., Perlman, H., Liu, H. and Pope, R.M., 2000. Macrophages require constitutive NFkappaB activation to maintain A1 expression and mitochondrial homeostasis, Mol Cell Biol. 20, 8855-65.

[30] Liu, H., Perlman, H., Pagliari, L.J. and Pope, R.M., 2001. Constitutively activated Akt-1 is vital for the survival of human monocyte-differentiated macrophages. Role of $\mathrm{Mcl}-1$, independent of nuclear factor (NF)-kappaB, Bad, or caspase activation, J Exp Med. 194, 113-26.

[31] Nagy, G., Daniel, B., Jonas, D., Nagy, L. and Barta, E., 2013. A novel method to predict regulatory regions based on histone mark landscapes in macrophages, Immunobiology. 218, 1416-27.

[32] Barta, E., 2011. Command line analysis of ChIP-seq results., EMBnet.Journal. 13-17.

[33] Li, H. and Durbin, R., 2009. Fast and accurate short read alignment with Burrows-Wheeler transform, Bioinformatics. 25, 1754-60.

[34] Heinz, S., Benner, C., Spann, N., Bertolino, E., Lin, Y.C., Laslo, P., Cheng, J.X., Murre, C., Singh, H. and Glass, C.K., 2010. Simple combinations of lineage-determining transcription factors prime cis-regulatory elements required for macrophage and B cell identities, Mol Cell. 38, 576-89.

[35] Thorvaldsdottir, H., Robinson, J.T. and Mesirov, J.P., 2013. Integrative Genomics Viewer (IGV): high-performance genomics data visualization and exploration, Brief Bioinform. 14, 178-92.

[36] Quinlan, A.R. and Hall, I.M., 2010. BEDTools: a flexible suite of utilities for comparing genomic features, Bioinformatics. 26, 841-2.

[37] Ross-Innes, C.S., Stark, R., Teschendorff, A.E., Holmes, K.A., Ali, H.R., Dunning, M.J., Brown, G.D., Gojis, O., Ellis, I.O., Green, A.R., Ali, S., Chin, S.F., Palmieri, C., Caldas, C. and Carroll, J.S., 2012. Differential oestrogen receptor binding is associated with clinical outcome in breast cancer, Nature. 481, 389-93.

[38] Kestler, H.A., Muller, A., Gress, T.M. and Buchholz, M., 2005. Generalized Venn diagrams: a new method of visualizing complex genetic set relations, Bioinformatics. 21, 1592-5.

[39] Trapnell, C., Hendrickson, D.G., Sauvageau, M., Goff, L., Rinn, J.L. and Pachter, L., 2013. Differential analysis of gene regulation at transcript resolution with RNA-seq, Nat Biotechnol. 31, 46-53.

[40] Team, R.D.C., 2013. A language and environment for statistical computing., R Foundation for Statistical Computing.

[41] Kolde, R., 2013. pheatmap: Pretty Heatmaps, R package version 0.7.7.

[42] Czimmerer, Z., Varga, T., Poliska, S., Nemet, I., Szanto, A. and Nagy, L., 2012. Identification of novel markers of alternative activation and potential endogenous PPARgamma ligand production mechanisms in human IL-4 stimulated differentiating macrophages, Immunobiology. 217, 130114.

[43] Martinez, F.O., Helming, L., Milde, R., Varin, A., Melgert, B.N., Draijer, C., Thomas, B., Fabbri, M., Crawshaw, A., Ho, L.P., Ten Hacken, N.H., Cobos Jimenez, V., Kootstra, N.A., Hamann, J., Greaves, D.R., Locati, M., Mantovani, A. and Gordon, S., 2013. Genetic programs expressed in resting and IL-4 alternatively activated mouse and human macrophages: similarities and differences, Blood. 121, e57-69. 
[44] Huang da, W., Sherman, B.T. and Lempicki, R.A., 2009. Systematic and integrative analysis of large gene lists using DAVID bioinformatics resources, Nat Protoc. 4, 44-57.

[45] Roszer, T., Menendez-Gutierrez, M.P., Cedenilla, M. and Ricote, M., 2013. Retinoid X receptors in macrophage biology, Trends Endocrinol Metab. 24, 460-8.

[46] Creyghton, M.P., Cheng, A.W., Welstead, G.G., Kooistra, T., Carey, B.W., Steine, E.J., Hanna, J., Lodato, M.A., Frampton, G.M., Sharp, P.A., Boyer, L.A., Young, R.A. and Jaenisch, R., 2010. Histone H3K27ac separates active from poised enhancers and predicts developmental state, Proc Natl Acad Sci U S A. 107, 21931-6.

[47] Simon, M.C., Olson, M., Scott, E., Hack, A., Su, G. and Singh, H., 1996. Terminal myeloid gene expression and differentiation requires the transcription factor PU.1, Curr Top Microbiol Immunol. 211, 113-9.

[48] Friedman, A.D., 2007. Transcriptional control of granulocyte and monocyte development, Oncogene. 26, 6816-28.

[49] Ghisletti, S., Barozzi, I., Mietton, F., Polletti, S., De Santa, F., Venturini, E., Gregory, L., Lonie, L., Chew, A., Wei, C.L., Ragoussis, J. and Natoli, G., 2010. Identification and characterization of enhancers controlling the inflammatory gene expression program in macrophages, Immunity. 32, 317-28.

[50] Pott, S., Kamrani, N.K., Bourque, G., Pettersson, S. and Liu, E.T., 2012. PPARG binding landscapes in macrophages suggest a genome-wide contribution of PU.1 to divergent PPARG binding in human and mouse, PLoS One. 7, e48102.

[51] Lefterova, M.I., Steger, D.J., Zhuo, D., Qatanani, M., Mullican, S.E., Tuteja, G., Manduchi, E., Grant, G.R. and Lazar, M.A., 2010. Cell-specific determinants of peroxisome proliferatoractivated receptor gamma function in adipocytes and macrophages, Mol Cell Biol. 30, 2078-89.

[52] Nielsen, R., Pedersen, T.A., Hagenbeek, D., Moulos, P., Siersbaek, R., Megens, E., Denissov, S., Borgesen, M., Francoijs, K.J., Mandrup, S. and Stunnenberg, H.G., 2008. Genome-wide profiling of PPARgamma:RXR and RNA polymerase II occupancy reveals temporal activation of distinct metabolic pathways and changes in RXR dimer composition during adipogenesis, Genes Dev. 22, 2953-67.

[53] Hamza, M.S., Pott, S., Vega, V.B., Thomsen, J.S., Kandhadayar, G.S., Ng, P.W., Chiu, K.P., Pettersson, S., Wei, C.L., Ruan, Y. and Liu, E.T., 2009. De-novo identification of PPARgamma/RXR binding sites and direct targets during adipogenesis, PLoS One. 4, e4907.

[54] Meyer, M.B., Goetsch, P.D. and Pike, J.W., 2010. Genome-wide analysis of the VDR/RXR cistrome in osteoblast cells provides new mechanistic insight into the actions of the vitamin D hormone, J Steroid Biochem Mol Biol. 121, 136-41.

[55] Elo, L.L., Jarvenpaa, H., Tuomela, S., Raghav, S., Ahlfors, H., Laurila, K., Gupta, B., Lund, R.J., Tahvanainen, J., Hawkins, R.D., Oresic, M., Lahdesmaki, H., Rasool, O., Rao, K.V., Aittokallio, T. and Lahesmaa, R., 2010. Genome-wide profiling of interleukin-4 and STAT6 transcription factor regulation of human Th2 cell programming, Immunity. 32, 852-62.

[56] Wei, L., Vahedi, G., Sun, H.W., Watford, W.T., Takatori, H., Ramos, H.L., Takahashi, H., Liang, J., Gutierrez-Cruz, G., Zang, C., Peng, W., O'Shea, J.J. and Kanno, Y., 2010. Discrete roles of STAT4 and STAT6 transcription factors in tuning epigenetic modifications and transcription during $T$ helper cell differentiation, Immunity. 32, 840-51.

[57] Colotta, F., Saccani, S., Giri, J.G., Dower, S.K., Sims, J.E., Introna, M. and Mantovani, A., 1996. Regulated expression and release of the IL-1 decoy receptor in human mononuclear phagocytes, J Immunol. 156, 2534-41.

[58] Scotton, C.J., Martinez, F.O., Smelt, M.J., Sironi, M., Locati, M., Mantovani, A. and Sozzani, S., 2005. Transcriptional profiling reveals complex regulation of the monocyte IL-1 beta system by IL-13, J Immunol. 174, 834-45. 
[59] Rebe, C., Raveneau, M., Chevriaux, A., Lakomy, D., Sberna, A.L., Costa, A., Bessede, G., Athias, A., Steinmetz, E., Lobaccaro, J.M., Alves, G., Menicacci, A., Vachenc, S., Solary, E., Gambert, P. and Masson, D., 2009. Induction of transglutaminase 2 by a liver $X$ receptor/retinoic acid receptor alpha pathway increases the clearance of apoptotic cells by human macrophages, Circ Res. 105, 393-401.

[60] Nagy, L., Saydak, M., Shipley, N., Lu, S., Basilion, J.P., Yan, Z.H., Syka, P., Chandraratna, R.A., Stein, J.P., Heyman, R.A. and Davies, P.J., 1996. Identification and characterization of a versatile retinoid response element (retinoic acid receptor response element-retinoid $X$ receptor response element) in the mouse tissue transglutaminase gene promoter, J Biol Chem. 271, 4355-65.

[61] Natoli, G. and Andrau, J.C., 2012. Noncoding transcription at enhancers: general principles and functional models, Annu Rev Genet. 46, 1-19.

[62] Simandi, Z., Horvath, A., Nagy, P. and Nagy, L., 2016. Prediction and Validation of Gene Regulatory Elements Activated During Retinoic Acid Induced Embryonic Stem Cell Differentiation, J Vis Exp.

[63] Daniel, B., Nagy, G. and Nagy, L., 2014. The intriguing complexities of mammalian gene regulation: how to link enhancers to regulated genes. Are we there yet?, FEBS Lett. 588, 237991.

[64] Simandi, Z., Horvath, A., Wright, L.C., Cuaranta-Monroy, I., De Luca, I., Karolyi, K., Sauer, S., Deleuze, J.F., Gudas, L.J., Cowley, S.M. and Nagy, L., 2016. OCT4 Acts as an Integrator of Pluripotency and Signal-Induced Differentiation, Mol Cell. 63, 647-61.

[65] Simandi, Z.H., A; Cuaranta-Monroy, I; Sauer, S; Deleuze, JF; Nagy, L, 2017. RXR heterodimers orchestrate transcriptional control of neurogenesis and cell fate specification, Molecular and Cellular Endocrinology. This issue. 
A

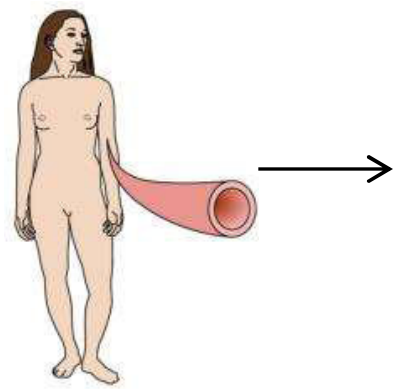

Buffy coat
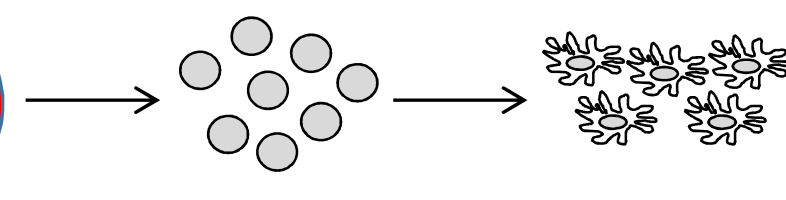

\section{象}

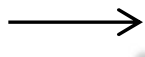

Veh

Magnetic separation of CD14+ monocytes

B
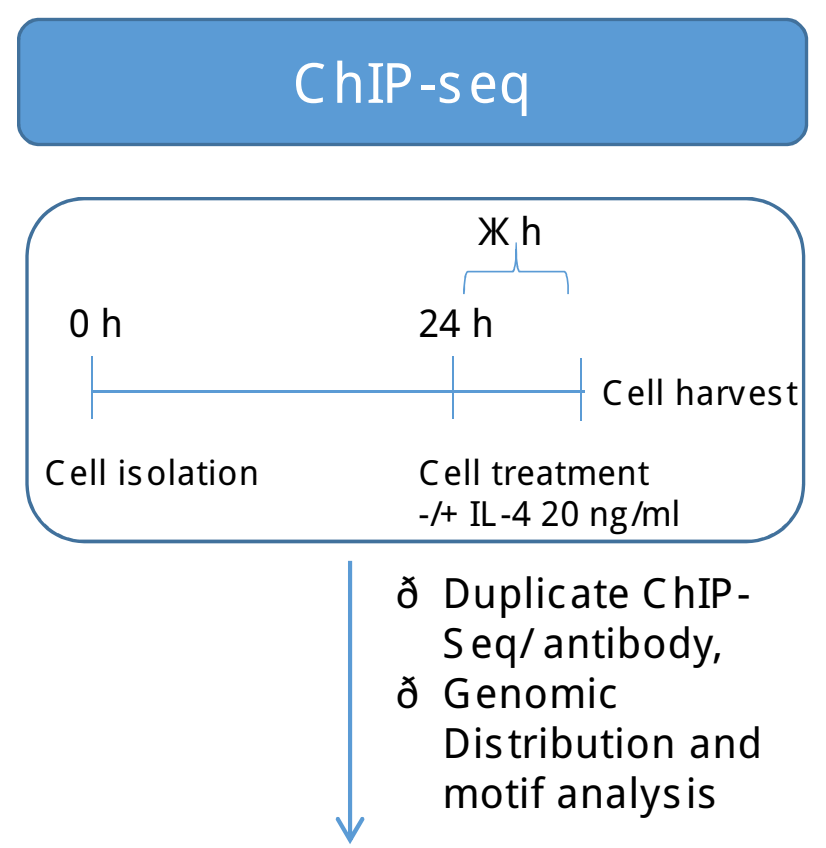

ð human macrophge-specific $\left(\mathrm{H} 3 \mathrm{~K} 27 \mathrm{Ac}+/ \mathrm{PU} .1^{+}\right)$enhancer set

ð STAT6 cistrome

ð RXR cistrome

ð RXR/STAT6 co-peaks
$24 \mathrm{~h}$ attachment on culture dish
Cell treatments

IL -4
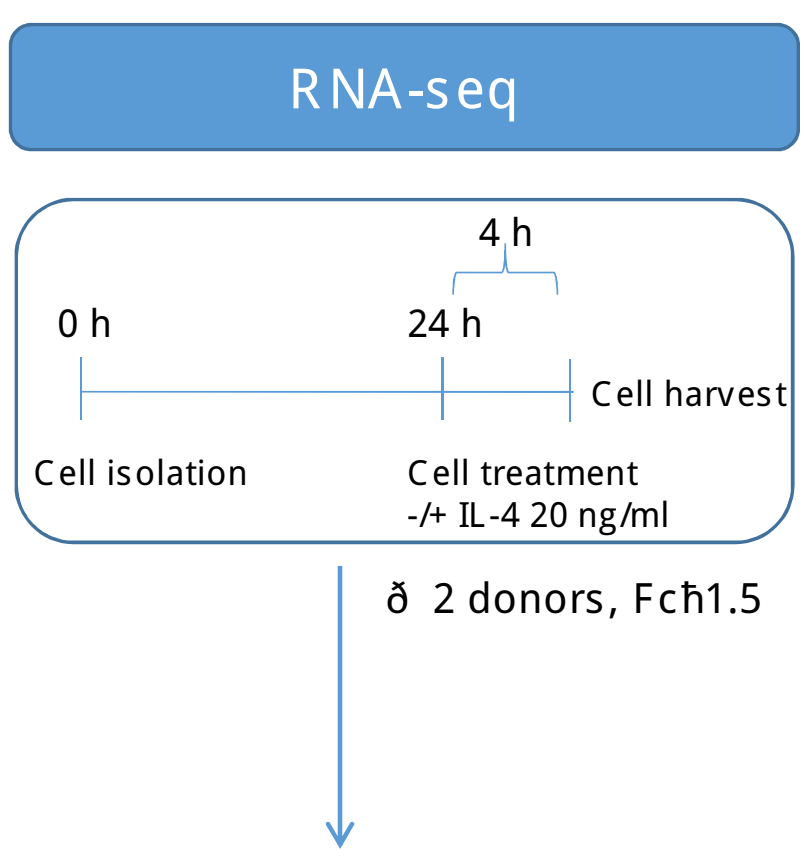
ðL-4-responsive macrophage
transcriptome 
A

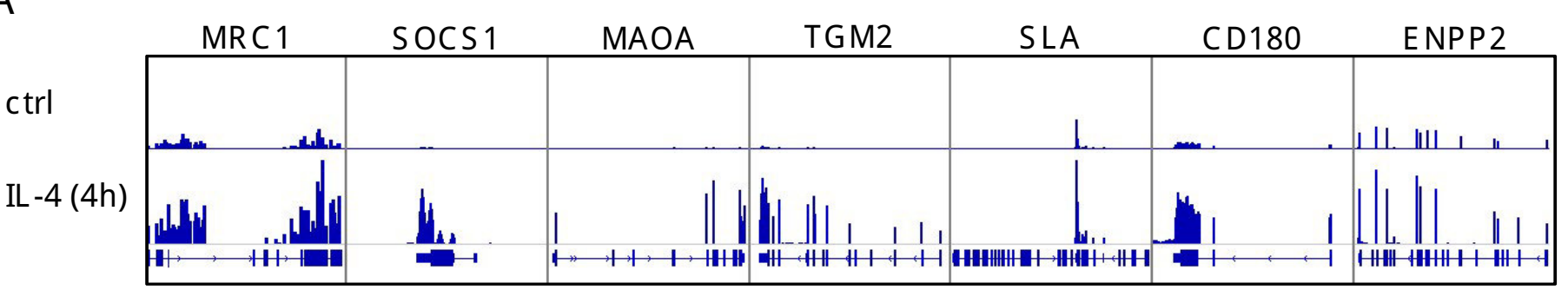

B

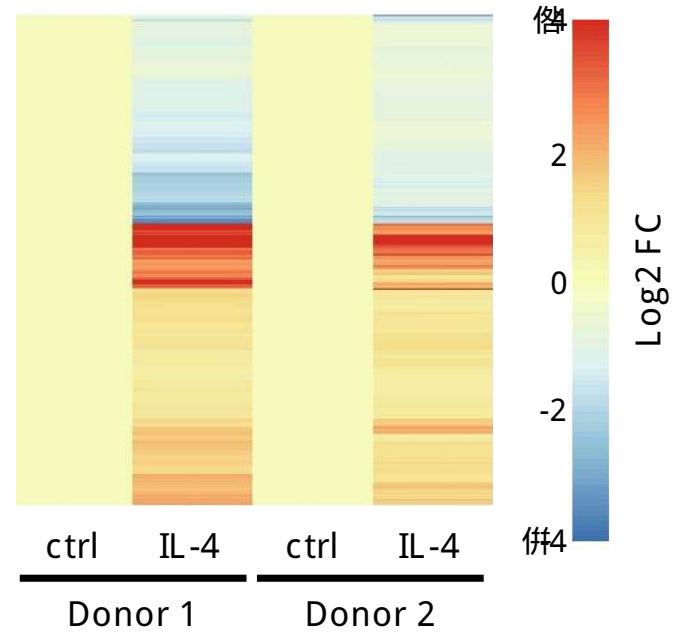

D

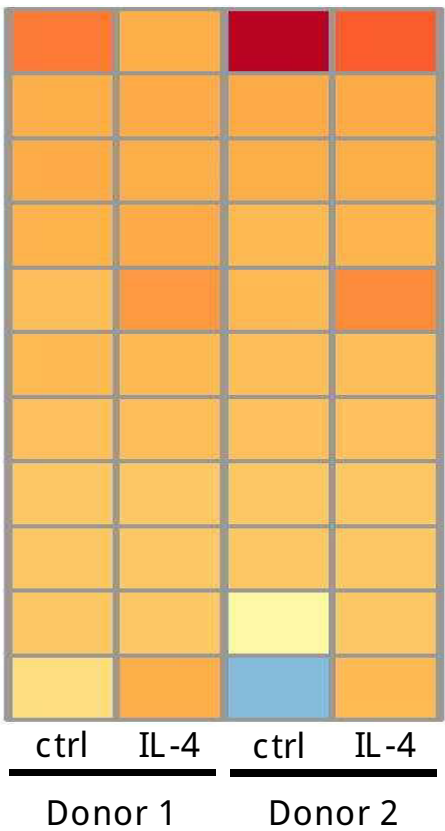

LXR

$\operatorname{LXR} \oint$

$\mathrm{RXR}$

PPAR 200

VDR

RXR $\oint$

RAR 100 주준

THR

PPAR 50

RAR $\therefore$

PPAR $\therefore$

C

\begin{tabular}{l}
1 \\
$\sim$ \\
0 \\
0 \\
\hline
\end{tabular}

Biological process Gene No. p-value FDR

$\begin{array}{cccc}\begin{array}{c}\text { inflammatory response } \\ \text { positive regulation of } \\ \text { inflammatory response } \\ \text { immune response }\end{array} & 56 & 1.27 \mathrm{E}-16 & 2.00 \mathrm{E}-13 \\ \begin{array}{c}\text { cellular response to } \\ \text { interferon-gamma } \\ \text { chemotaxis }\end{array} & 16 & 6.12 \mathrm{E}-11 & 1.11 \mathrm{E}-07 \\ \begin{array}{c}\text { chemokine-mediated } \\ \text { signaling pathway }\end{array} & 16 & 3.41 \mathrm{E}-09 & 6.20 \mathrm{E}-06 \\ \begin{array}{c}\text { response to } \\ \text { lipopolysaccharide } \\ \text { signal transduction }\end{array} & 24 & 1.33 \mathrm{E}-07 & 2.41 \mathrm{E}-04 \\ \text { monocyte chemotaxis } & 12 & 9.89 \mathrm{E}-07 & 4.03 \mathrm{E}-04 \\ \begin{array}{c}\text { lymphocyte chemotaxis } \\ \text { lym }-06\end{array} & 10 & 1.01 \mathrm{E}-06 & 1.83 \mathrm{E}-03\end{array}$


A

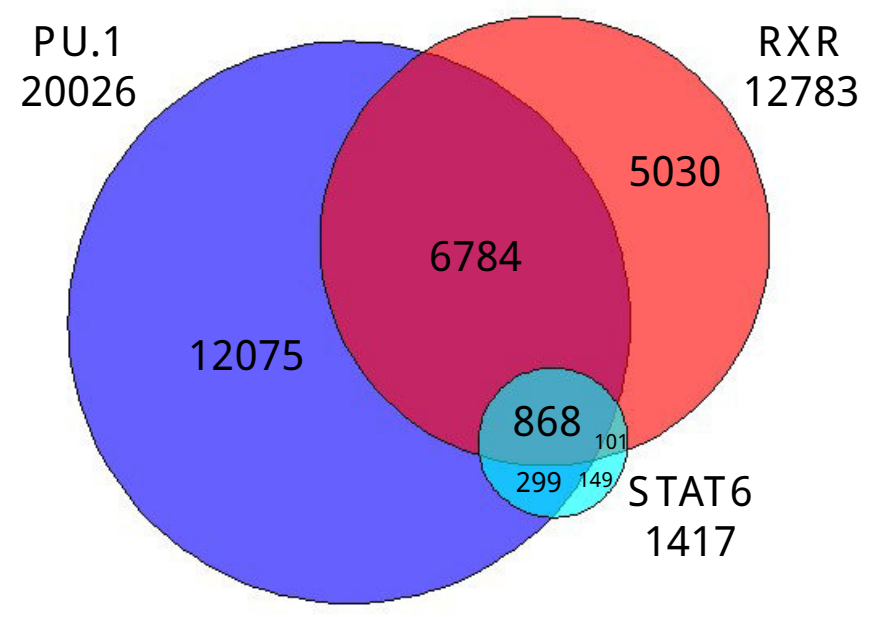

\begin{tabular}{|l|c|l|l|}
\hline Peaks (DiffBind) & PU.1 & RXR & STAT6 \\
\hline Consensus & 26531 & 14125 & 1573 \\
\hline Active (H3K27ac) & 20046 & 12799 & 1417 \\
\hline
\end{tabular}

C

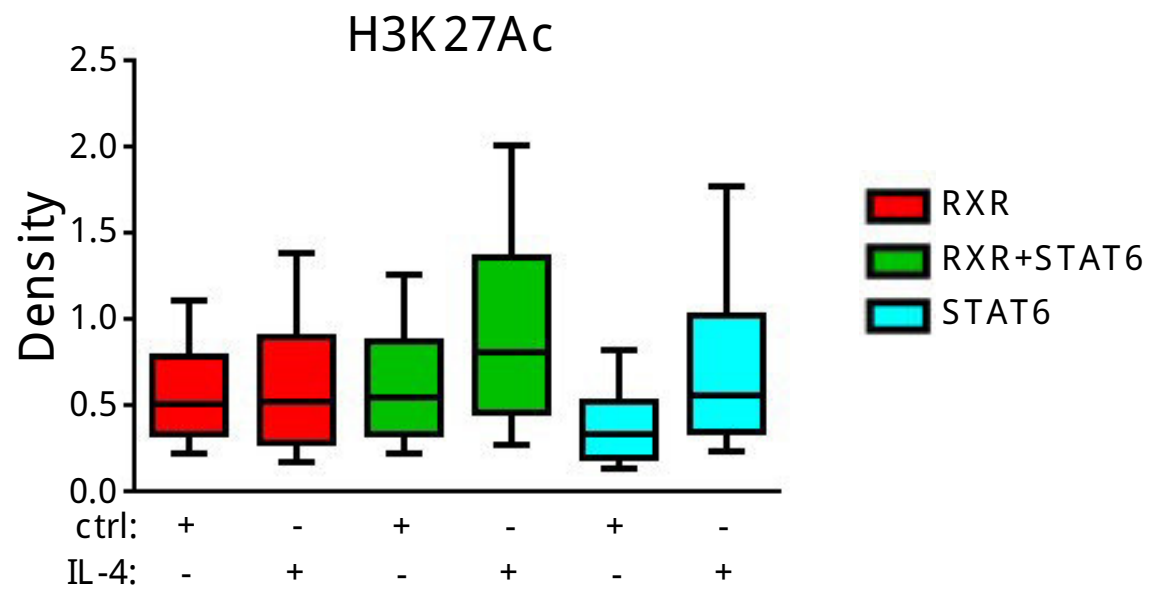

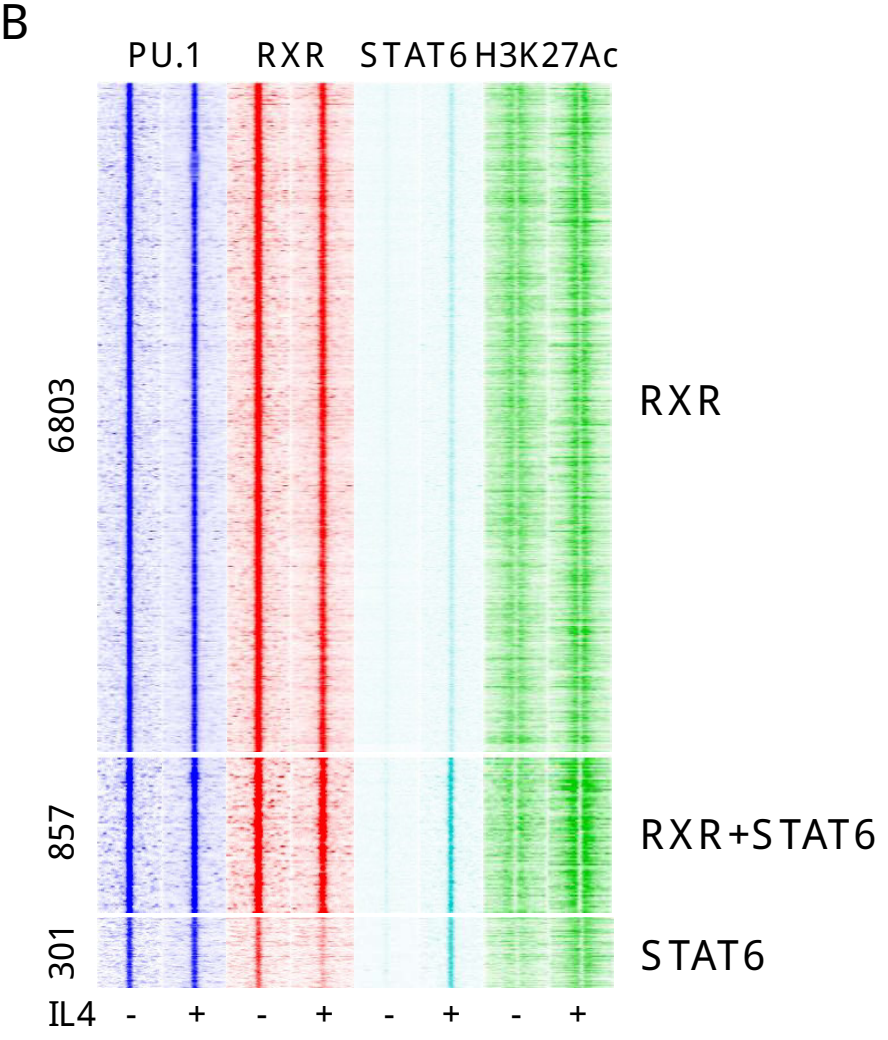


A
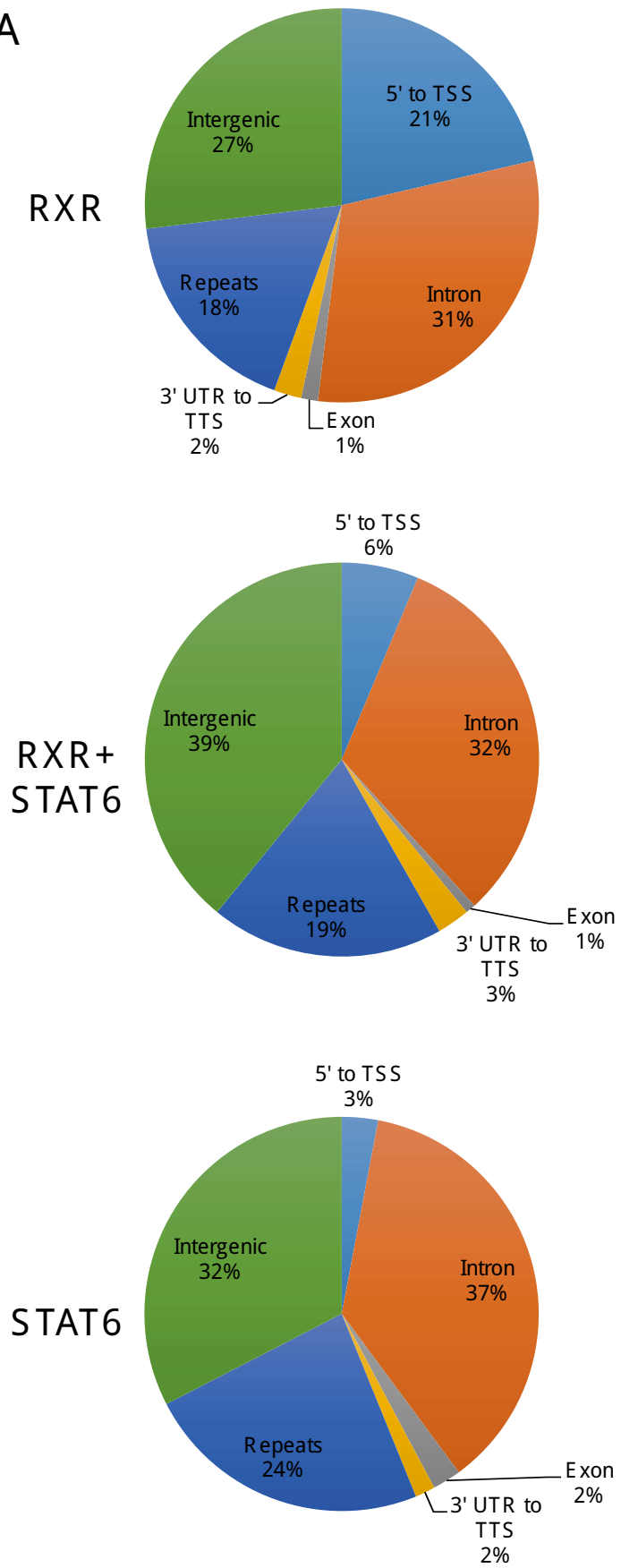

B

\begin{tabular}{|c|c|c|c|c|}
\hline \multicolumn{5}{|c|}{$\begin{array}{l}\text { PU.1 + RXR } \\
(883 / 1000)\end{array}$} \\
\hline & p-value & Target (\%) & $\mathrm{Bg}(\%)$ & Motif \\
\hline$\therefore=G G A A s x$ & $1 \mathrm{E}-278$ & 63.19 & 12.12 & PU.1 \\
\hline$T_{x} x_{x} \mathrm{CAA}$ & $1 \mathrm{E}-75$ & 16.53 & 2.31 & C/EBP \\
\hline$G G T T A C T=E G G T C A=$ & $1 \mathrm{E}-71$ & 2.94 & 0 & DR4 \\
\hline$T_{\subseteq A_{\varepsilon}} T C A$ & $1 \mathrm{E}-62$ & 20.84 & 4.78 & AP-1 \\
\hline$=\pi G A G_{C} T C A$ & $1 \mathrm{E}-41$ & 27.75 & 11.09 & NR half \\
\hline \multicolumn{5}{|c|}{$\begin{array}{c}\text { PU.1 + RXR + STAT 6 } \\
(703 / 857)\end{array}$} \\
\hline & p-value & Target (\%) & $\mathrm{Bg}(\%)$ & Motif \\
\hline$\ldots G G A A_{-T}$ & $1 \mathrm{E}-247$ & 52.54 & 6.21 & PU.1 \\
\hline $\mathrm{TT}_{\mathrm{a}}^{\mathrm{a} A}$ & $1 E-124$ & 36.76 & 6.31 & C/EBP \\
\hline TTc $=\mathrm{GAA}$ & $1 \mathrm{E}-92$ & 36.48 & 8.63 & STAT6 \\
\hline$=$ TGAeTCA & $1 E-38$ & 20.99 & 6.2 & AP-1 \\
\hline$G G G_{A \cap T T T} T C_{c}$ & $1 E-26$ & 21.27 & 8.28 & REL \\
\hline$\Rightarrow A A C C A C$ & $1 \mathrm{E}-22$ & 10.99 & 2.86 & RUNX \\
\hline \multicolumn{5}{|c|}{$\begin{array}{l}\text { PU.1 + STAT } 6 \\
(233 / 301)\end{array}$} \\
\hline & p-value & Target (\%) & $\mathrm{Bg}(\%)$ & Motif \\
\hline$G G A A_{\cong} T$ & $1 \mathrm{E}-80$ & 61.8 & 10.25 & PU.1 \\
\hline$-T=C_{z}=G A A_{-}$ & $1 \mathrm{E}-57$ & 45.06 & 6.96 & STAT6 \\
\hline${ }_{\triangle} T_{\mathrm{sT}} \mathrm{G}_{s} \mathrm{AA} I$ & $1 \mathrm{E}-22$ & 28.76 & 7.12 & $C / E B P$ \\
\hline a TGA ICA $=$ & $1 \mathrm{E}-18$ & 16.74 & 2.72 & $A P-1$ \\
\hline
\end{tabular}


A

IL -4 responsive genes-associated

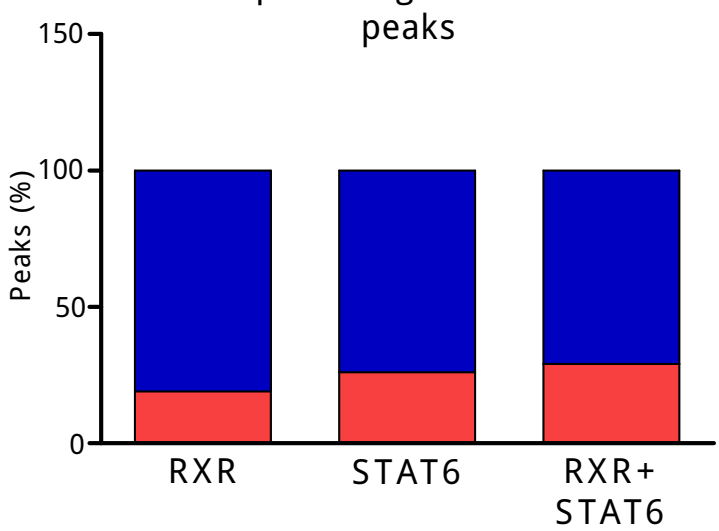

B

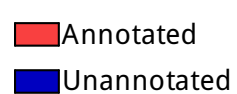

Peak distribution relative to TSS

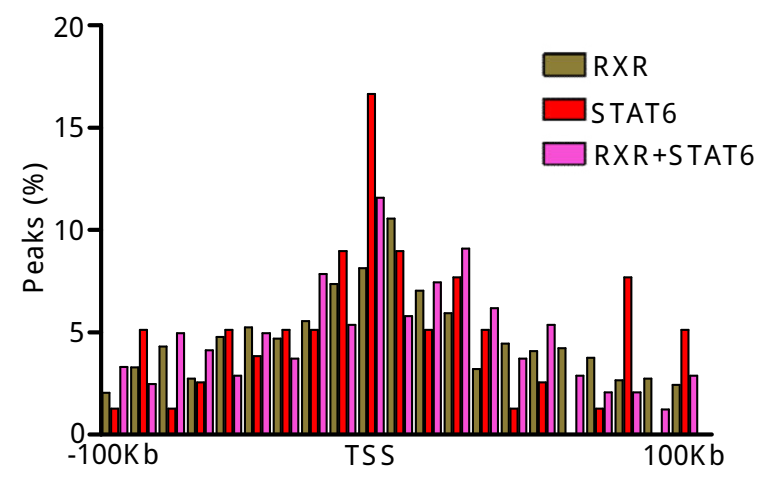

C

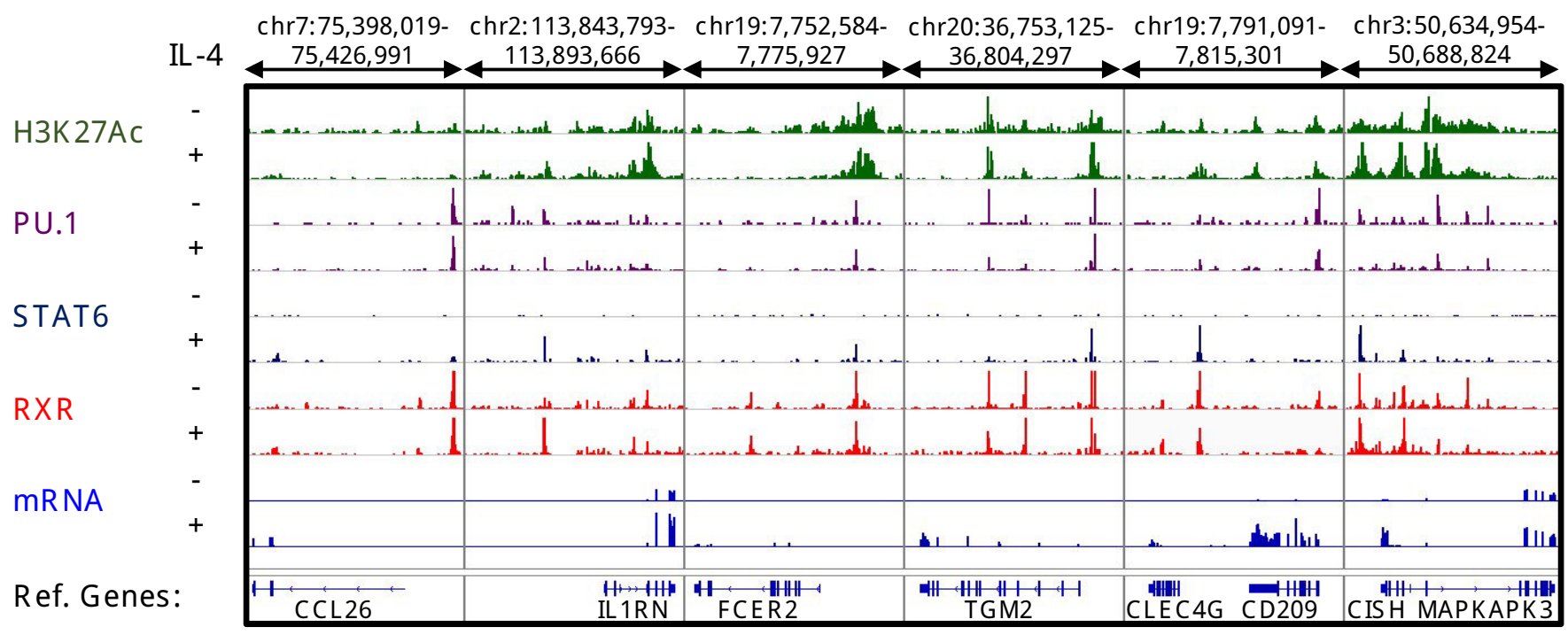


A

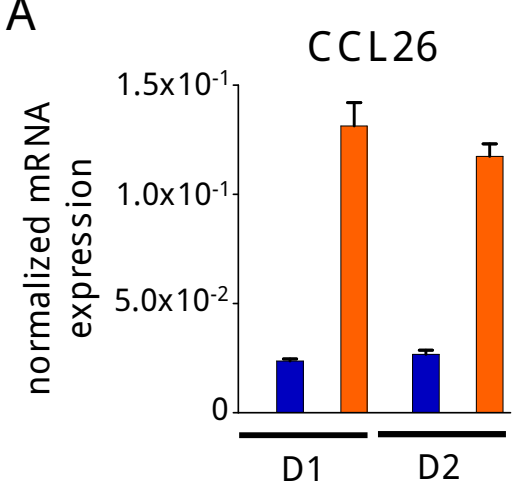

IL 1 R N

FCER2 TGM2
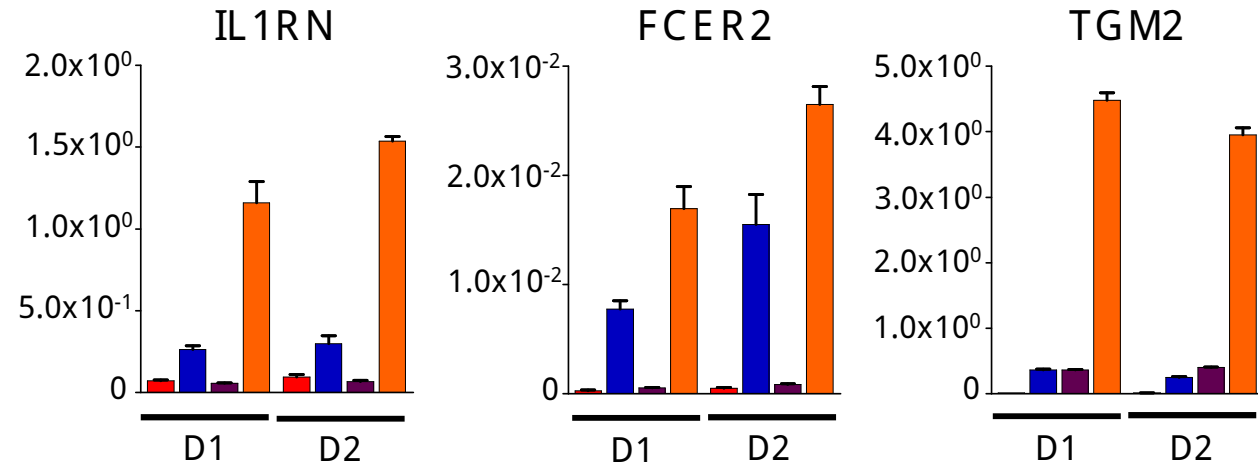

CLEC4G

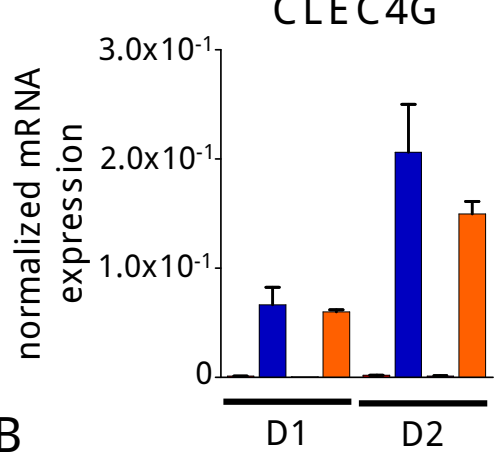

CD209
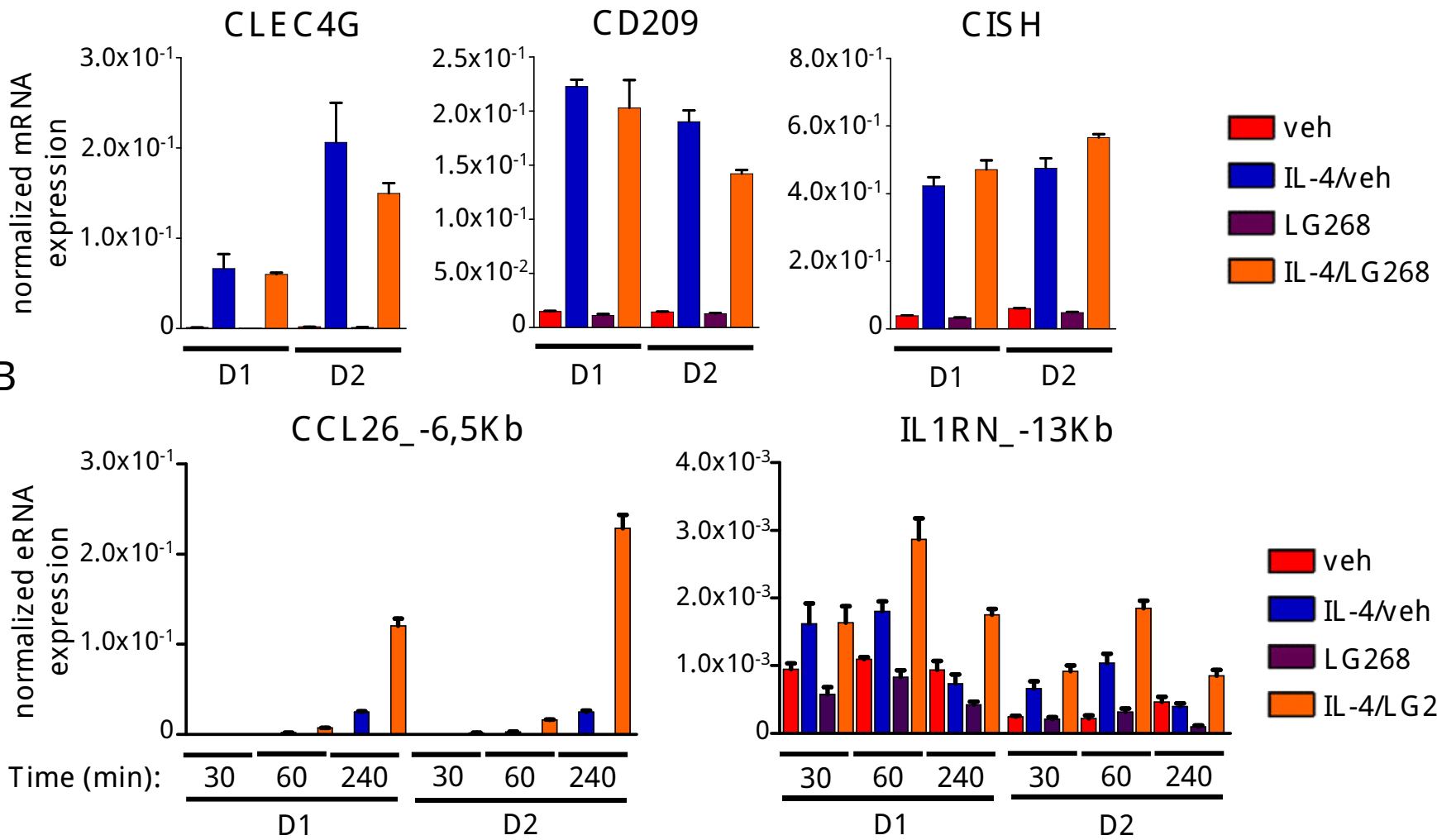

IL $1 R N_{-}-13 K b$

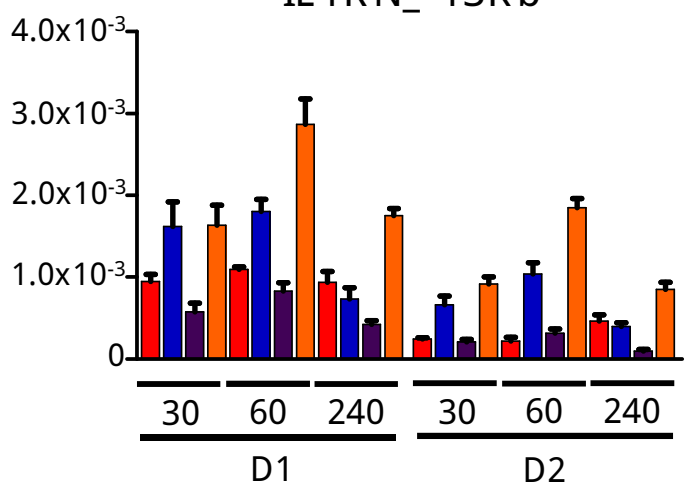

$\square$ veh

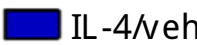

$\square$ LG268

$\square$ IL -4/L G 268

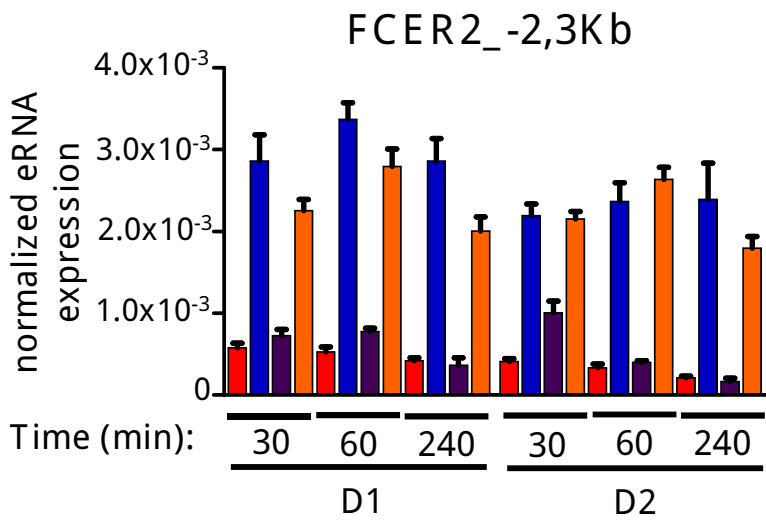

TGM2_-3,3Kb
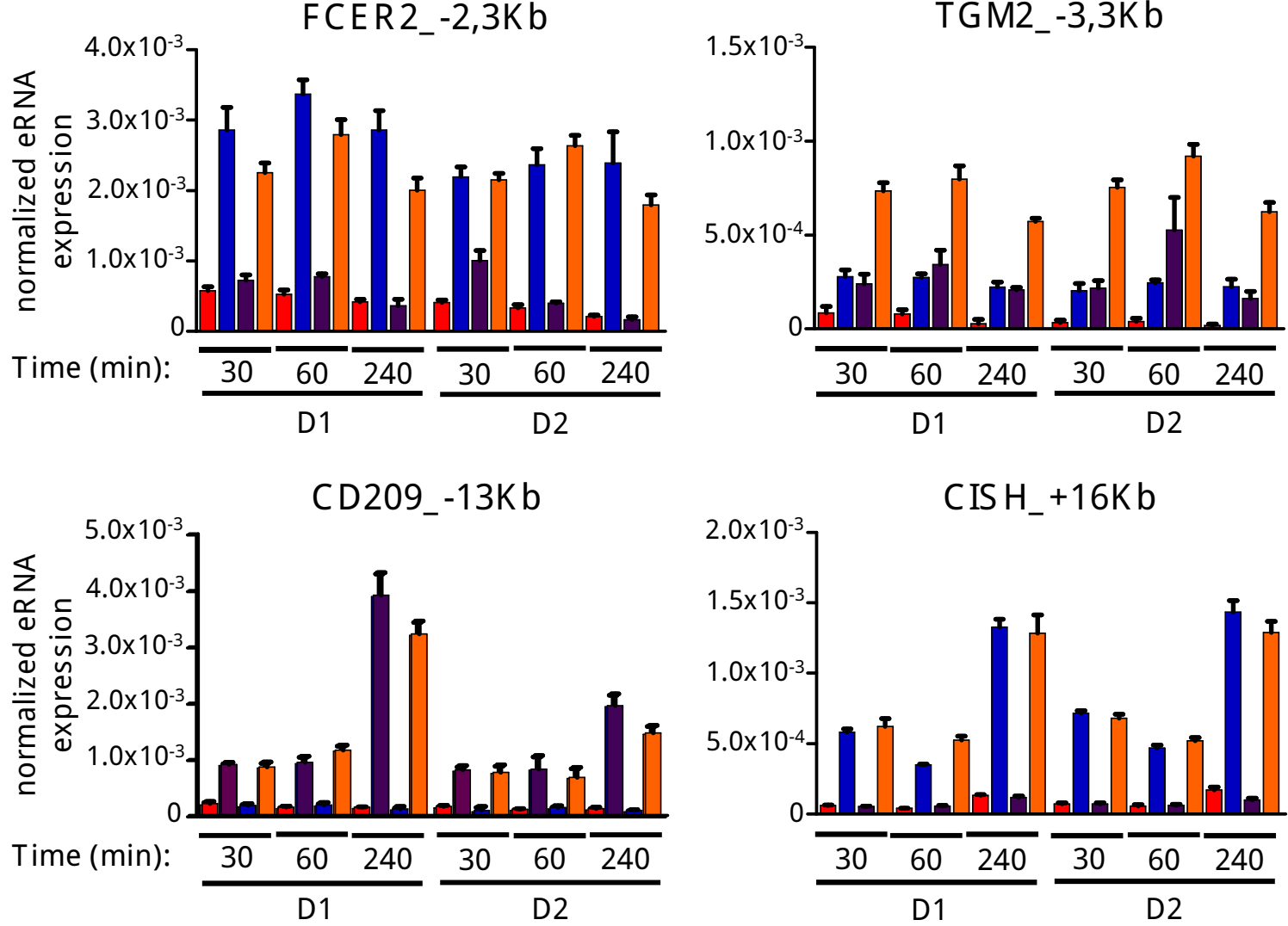
synergistic

potentiating

liganded RXR independent

veh
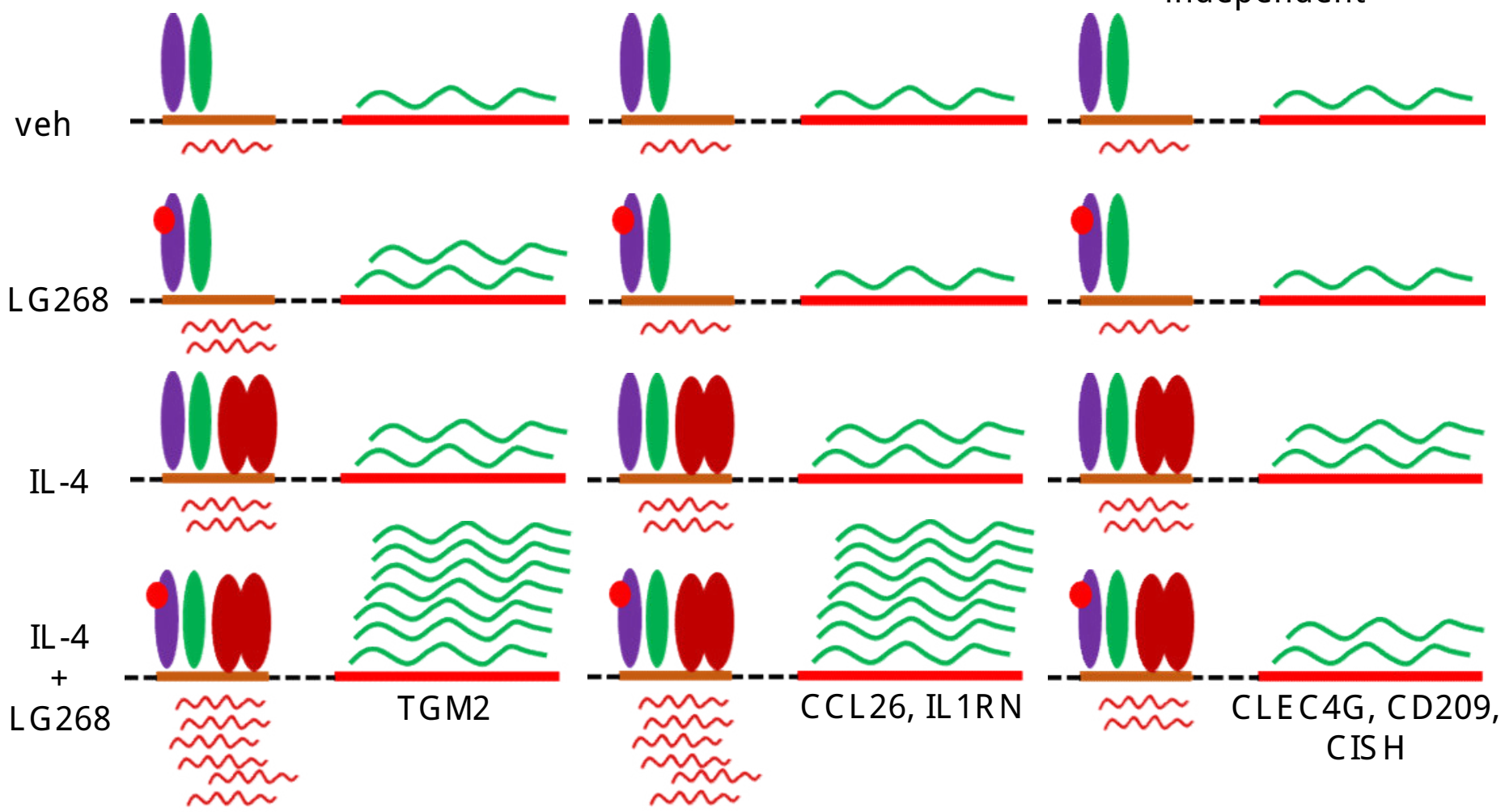

$\sim$ mRNA $\sim$ eRNA RXR NRR STAT6 LG268 
A

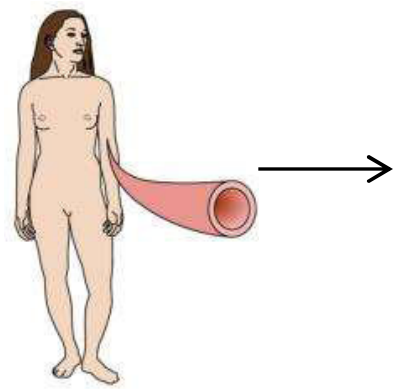

Buffy coat
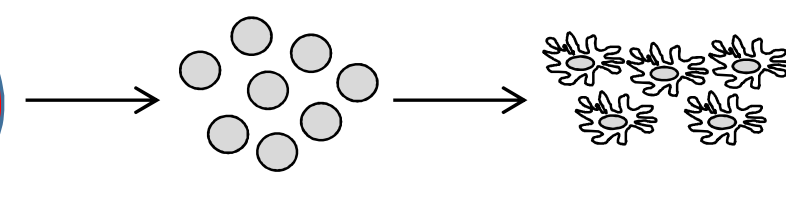

\section{象}

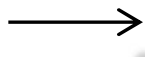

Veh

Magnetic separation of CD14+ monocytes

B
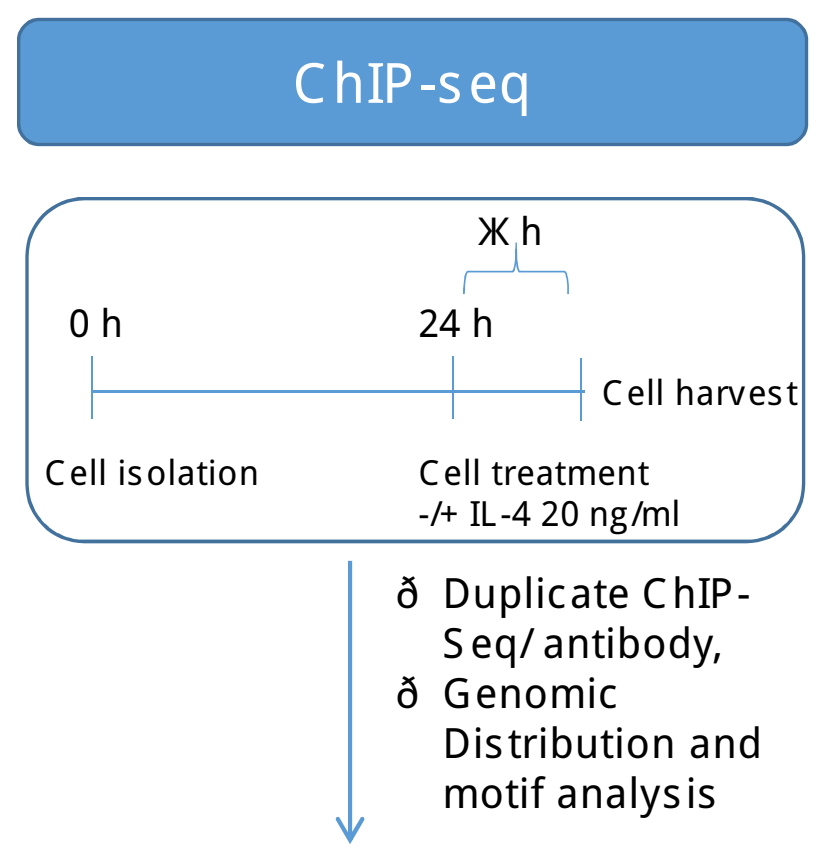

ð human macrophge-specific $\left(\mathrm{H} 3 \mathrm{~K} 27 \mathrm{Ac}+/ \mathrm{PU} .1^{+}\right)$enhancer set

ð STAT6 cistrome

ð RXR cistrome

ð RXR/STAT6 co-peaks
$24 \mathrm{~h}$ attachment on culture dish
Cell treatments

IL -4
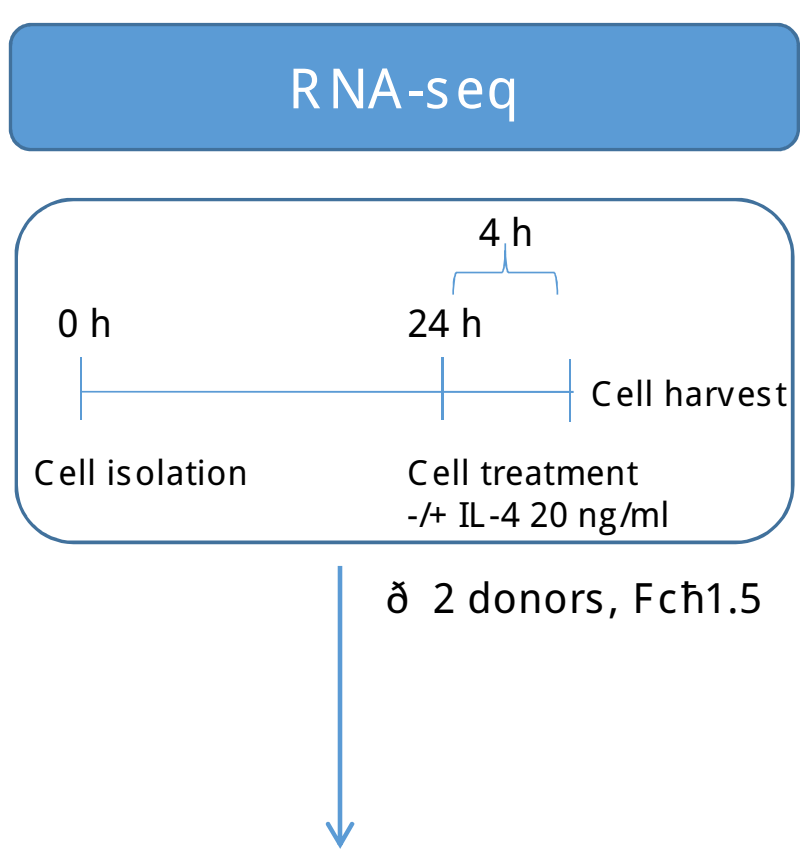
ðL-4-responsive macrophage
transcriptome 
A

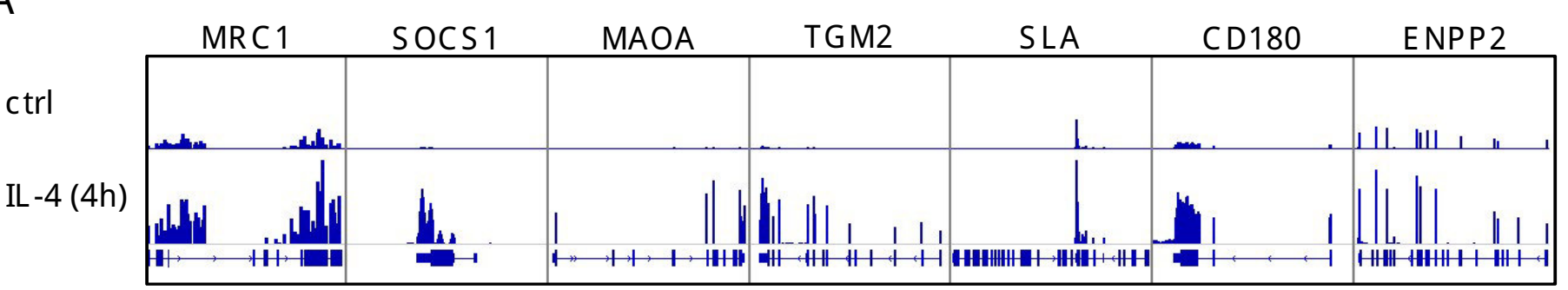

B

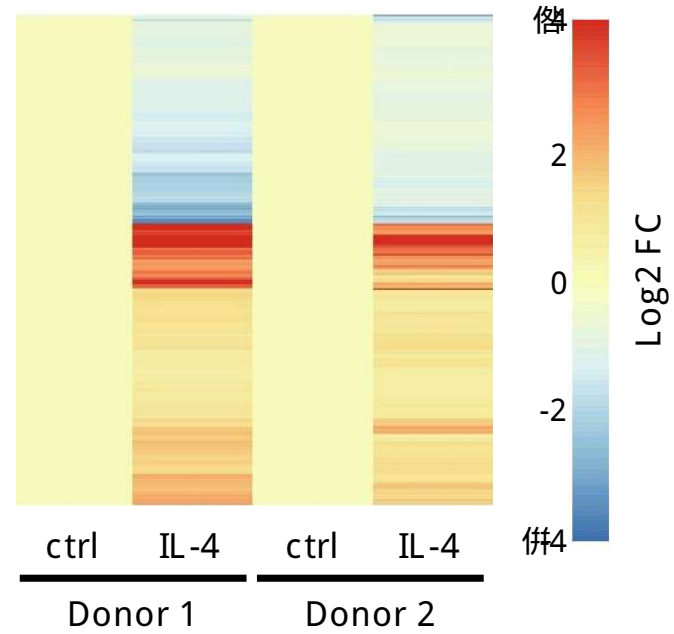

D

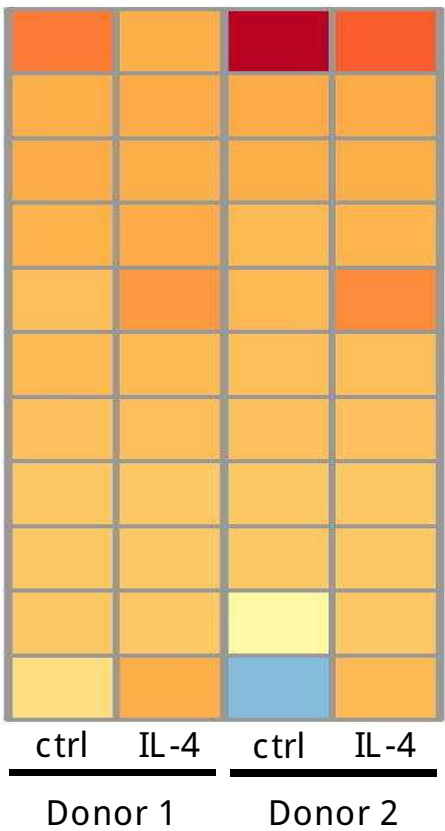

LXR

$\operatorname{LXR} \oint$

$\mathrm{RXR}$

PPAR 200

VDR

RXR $\oint$

RAR 100 주준

THR

PPAR 50

RAR $\therefore$

PPAR $\therefore$

C

\begin{tabular}{l}
1 \\
$\sim$ \\
0 \\
0 \\
\hline
\end{tabular}

Biological process Gene No. p-value FDR

$\begin{array}{cccc}\begin{array}{c}\text { inflammatory response } \\ \text { positive regulation of } \\ \text { inflammatory response } \\ \text { immune response }\end{array} & 56 & 1.27 \mathrm{E}-16 & 2.00 \mathrm{E}-13 \\ \begin{array}{c}\text { cellular response to } \\ \text { interferon-gamma } \\ \text { chemotaxis }\end{array} & 16 & 6.12 \mathrm{E}-11 & 1.11 \mathrm{E}-07 \\ \begin{array}{c}\text { chemokine-mediated } \\ \text { signaling pathway }\end{array} & 16 & 3.41 \mathrm{E}-09 & 6.20 \mathrm{E}-06 \\ \begin{array}{c}\text { response to } \\ \text { lipopolysaccharide } \\ \text { signal transduction }\end{array} & 24 & 1.33 \mathrm{E}-07 & 2.41 \mathrm{E}-04 \\ \text { monocyte chemotaxis } & 12 & 9.89 \mathrm{E}-07 & 4.03 \mathrm{E}-04 \\ \begin{array}{c}\text { lymphocyte chemotaxis } \\ \text { lym }-06\end{array} & 10 & 1.01 \mathrm{E}-06 & 1.83 \mathrm{E}-03\end{array}$


A

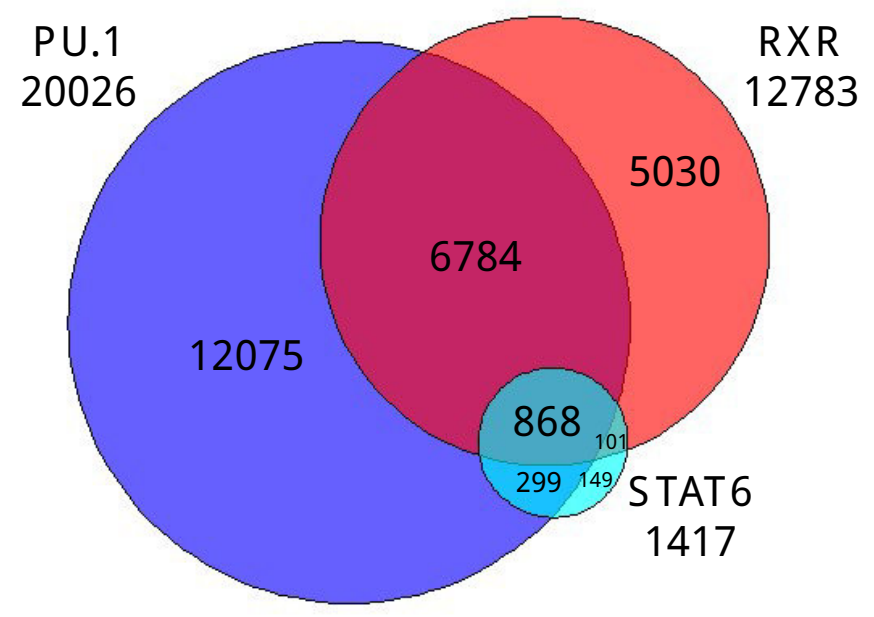

\begin{tabular}{|l|c|l|l|}
\hline Peaks (DiffBind) & PU.1 & RXR & STAT6 \\
\hline Consensus & 26531 & 14125 & 1573 \\
\hline Active (H3K27ac) & 20046 & 12799 & 1417 \\
\hline
\end{tabular}

C

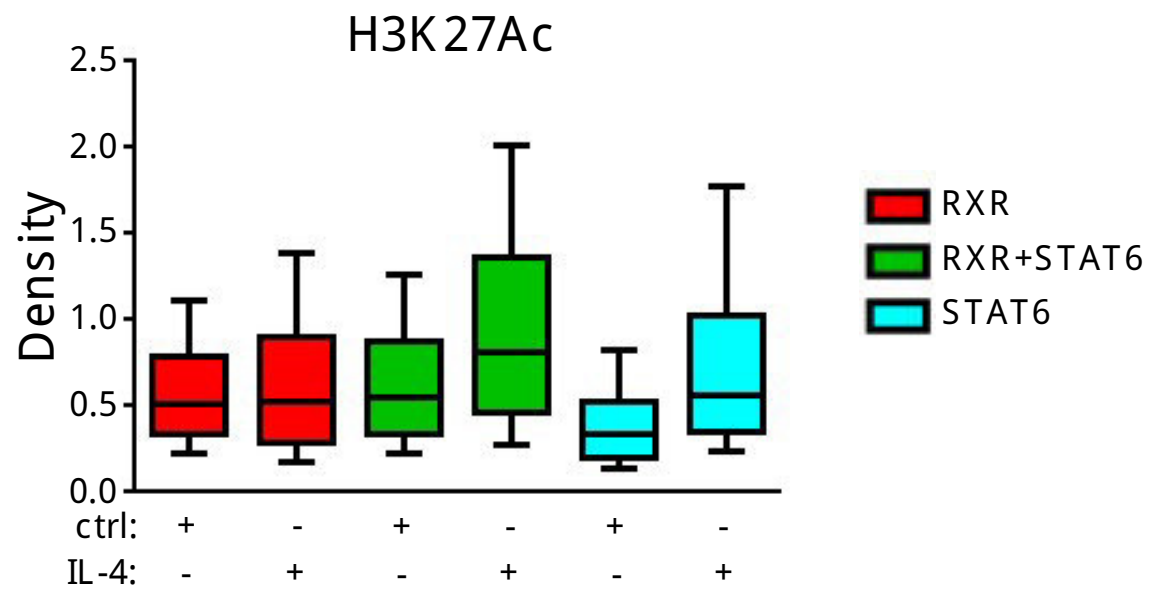

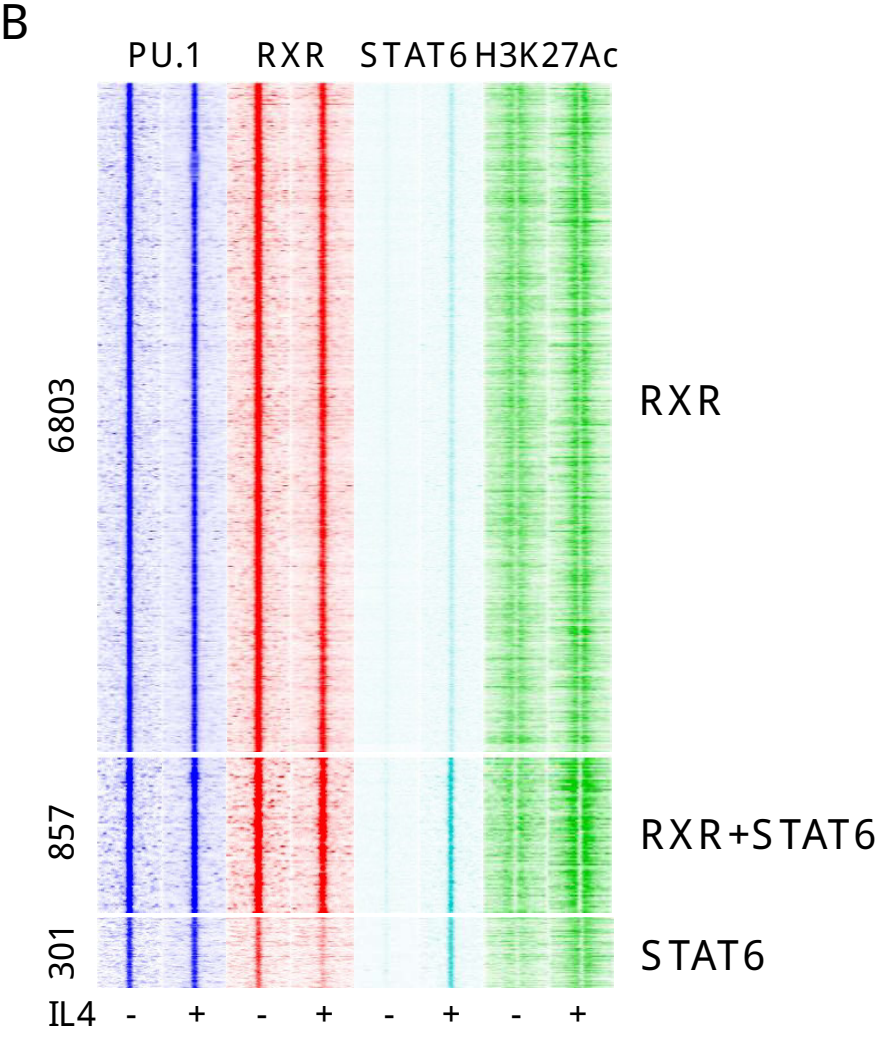


A
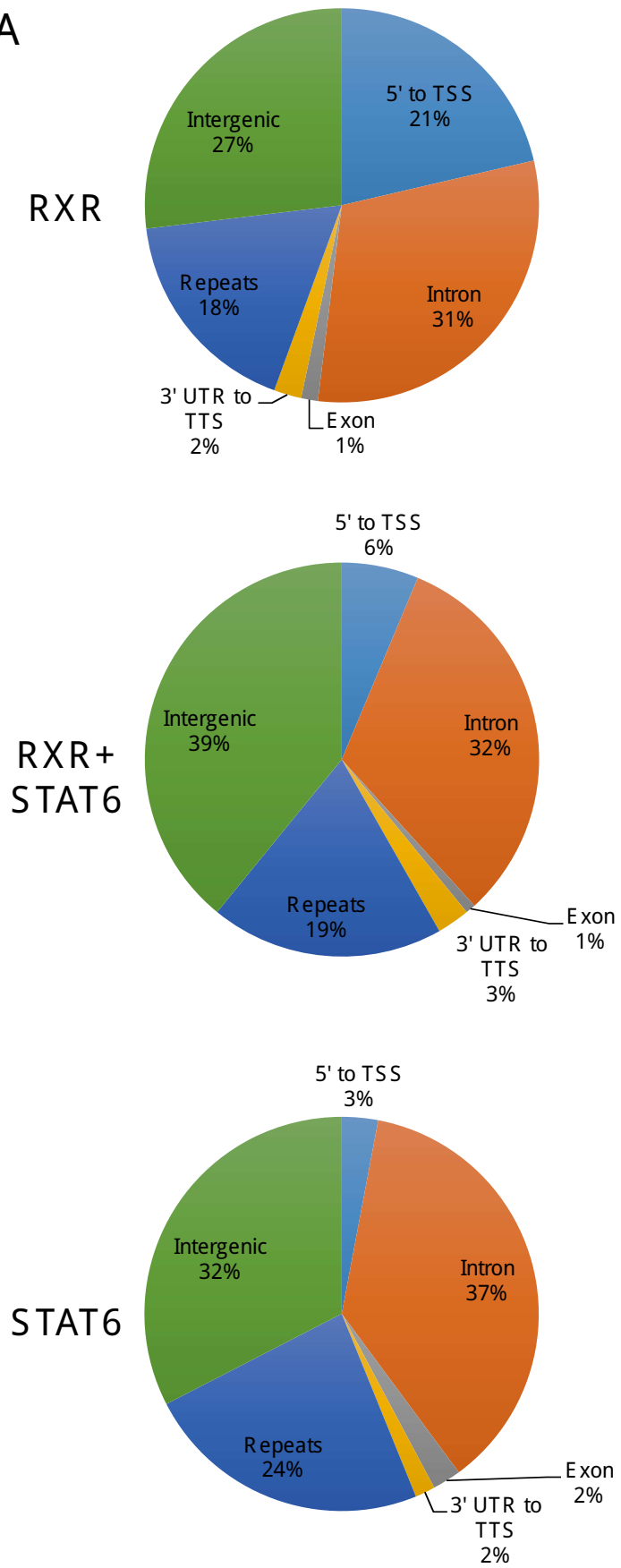

B

\begin{tabular}{|c|c|c|c|c|}
\hline \multicolumn{5}{|c|}{$\begin{array}{l}\text { PU.1 + RXR } \\
(883 / 1000)\end{array}$} \\
\hline & p-value & Target (\%) & $\mathrm{Bg}(\%)$ & Motif \\
\hline$\therefore=G G A A s x$ & $1 \mathrm{E}-278$ & 63.19 & 12.12 & PU.1 \\
\hline$T_{x} x_{x} \mathrm{CAA}$ & $1 \mathrm{E}-75$ & 16.53 & 2.31 & C/EBP \\
\hline$G G T T A C T=E G G T C A=$ & $1 \mathrm{E}-71$ & 2.94 & 0 & DR4 \\
\hline$T_{\subseteq A_{\varepsilon}} T C A$ & $1 \mathrm{E}-62$ & 20.84 & 4.78 & AP-1 \\
\hline$=\pi G A G_{C} T C A$ & $1 \mathrm{E}-41$ & 27.75 & 11.09 & NR half \\
\hline \multicolumn{5}{|c|}{$\begin{array}{c}\text { PU.1 + RXR + STAT 6 } \\
(703 / 857)\end{array}$} \\
\hline & p-value & Target (\%) & $\mathrm{Bg}(\%)$ & Motif \\
\hline$\ldots G G A A_{-T}$ & $1 \mathrm{E}-247$ & 52.54 & 6.21 & PU.1 \\
\hline $\mathrm{TT}_{\mathrm{a}}^{\mathrm{a} A}$ & $1 E-124$ & 36.76 & 6.31 & C/EBP \\
\hline TTc $=\mathrm{GAA}$ & $1 \mathrm{E}-92$ & 36.48 & 8.63 & STAT6 \\
\hline$=$ TGAeTCA & $1 E-38$ & 20.99 & 6.2 & AP-1 \\
\hline$G G G_{A \cap T T T} T C_{c}$ & $1 E-26$ & 21.27 & 8.28 & REL \\
\hline$\Rightarrow A A C C A C$ & $1 \mathrm{E}-22$ & 10.99 & 2.86 & RUNX \\
\hline \multicolumn{5}{|c|}{$\begin{array}{l}\text { PU.1 + STAT } 6 \\
(233 / 301)\end{array}$} \\
\hline & p-value & Target (\%) & $\mathrm{Bg}(\%)$ & Motif \\
\hline$G G A A_{\cong} T$ & $1 \mathrm{E}-80$ & 61.8 & 10.25 & PU.1 \\
\hline$-T=C_{z}=G A A_{-}$ & $1 \mathrm{E}-57$ & 45.06 & 6.96 & STAT6 \\
\hline${ }_{\triangle} T_{\mathrm{sT}} \mathrm{G}_{s} \mathrm{AA} I$ & $1 \mathrm{E}-22$ & 28.76 & 7.12 & $C / E B P$ \\
\hline a TGA ICA $=$ & $1 \mathrm{E}-18$ & 16.74 & 2.72 & $A P-1$ \\
\hline
\end{tabular}


A

IL -4 responsive genes-associated

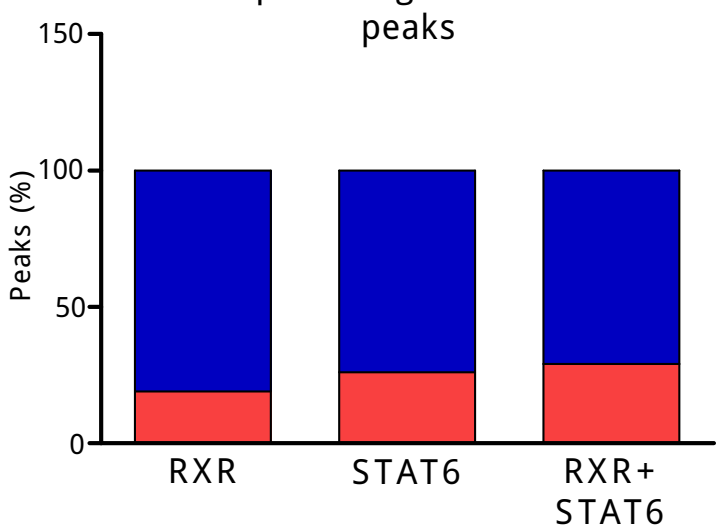

B

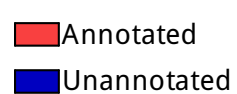

Peak distribution relative to TSS

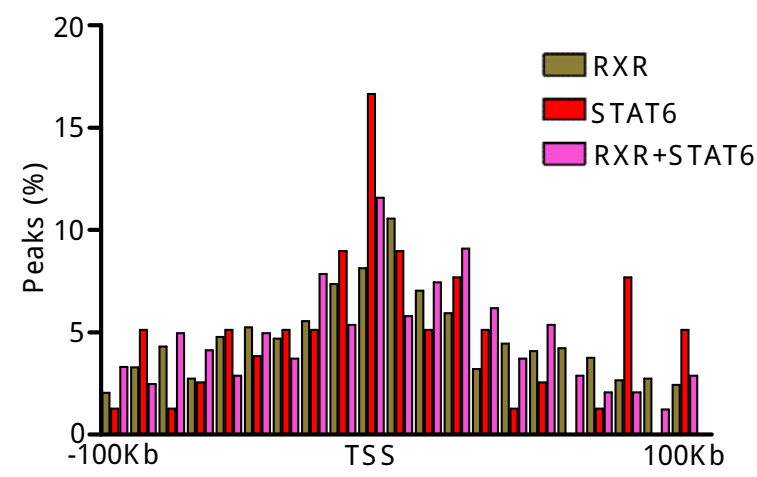

C

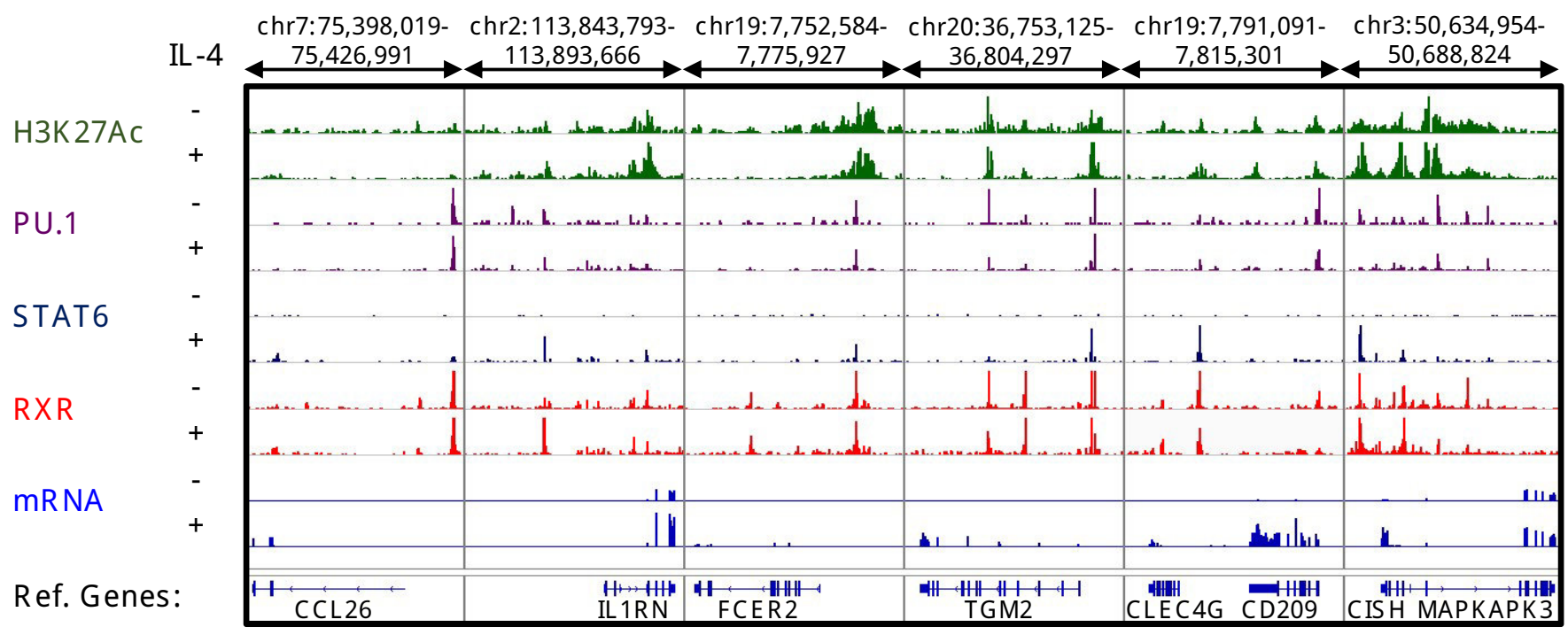


A

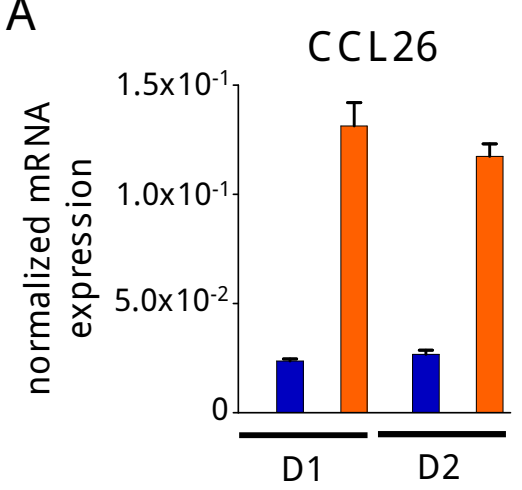

IL 1 R N

FCER2 TGM2
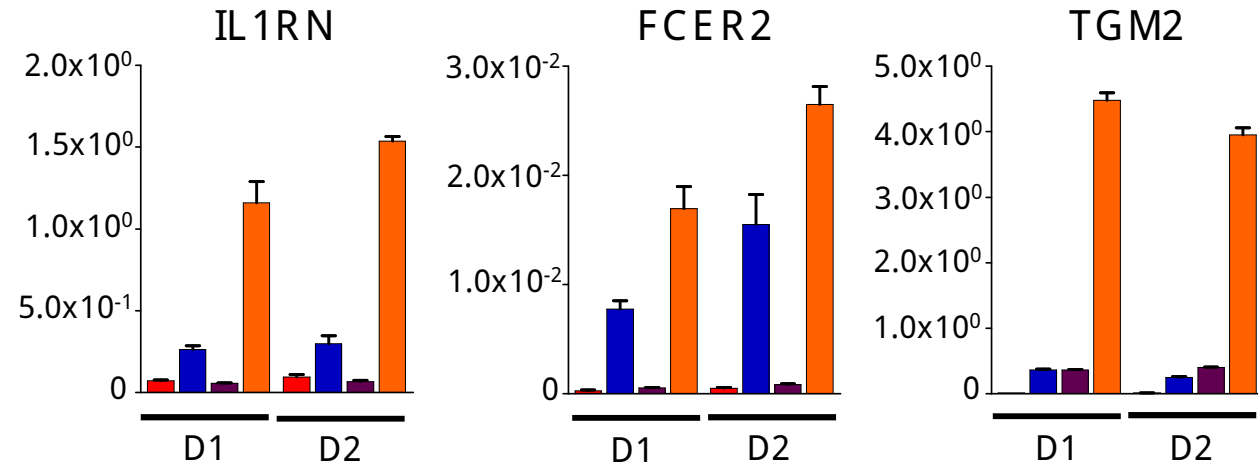

CLEC4G

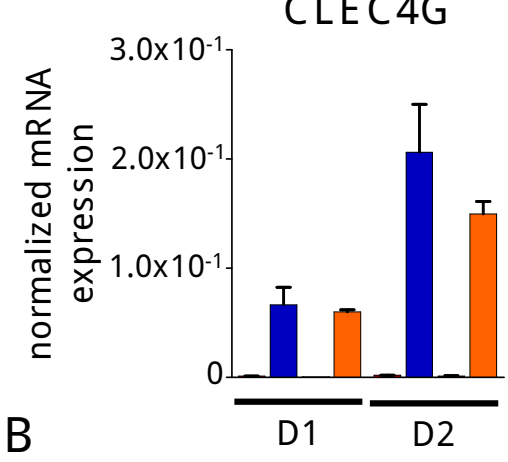

CD209
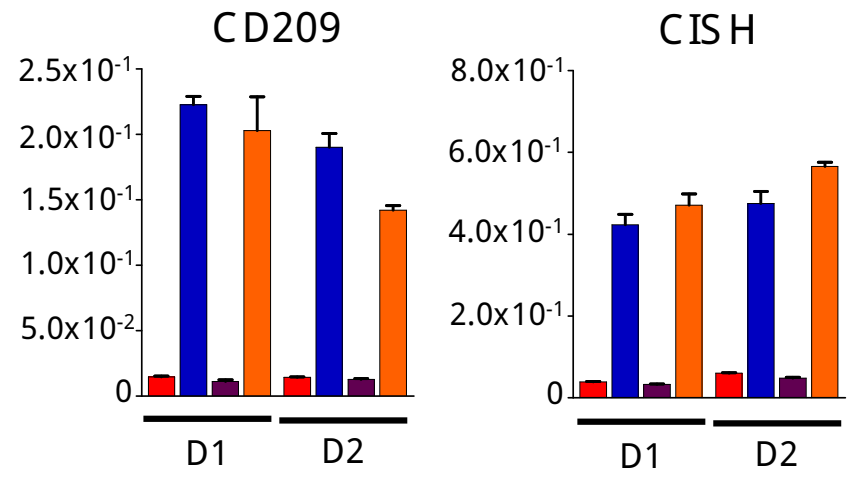

$\square$ veh

CCL26_-6,5Kb

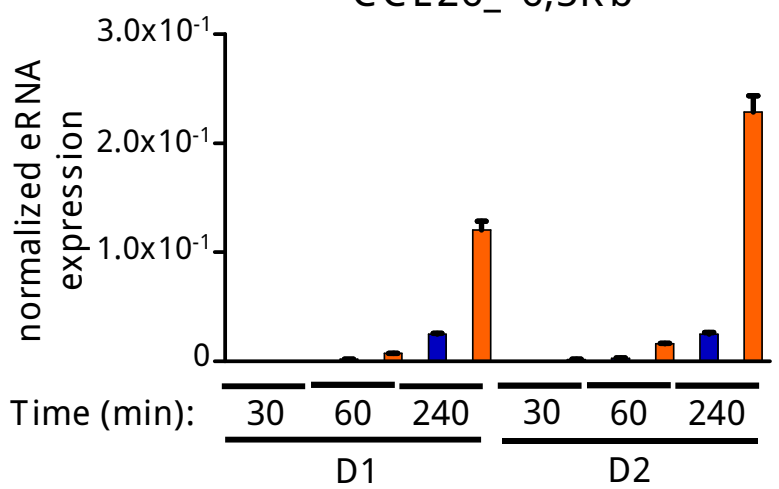

IL $1 R N_{-}-13 K b$

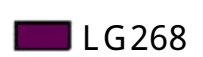

IL-4/L G 268

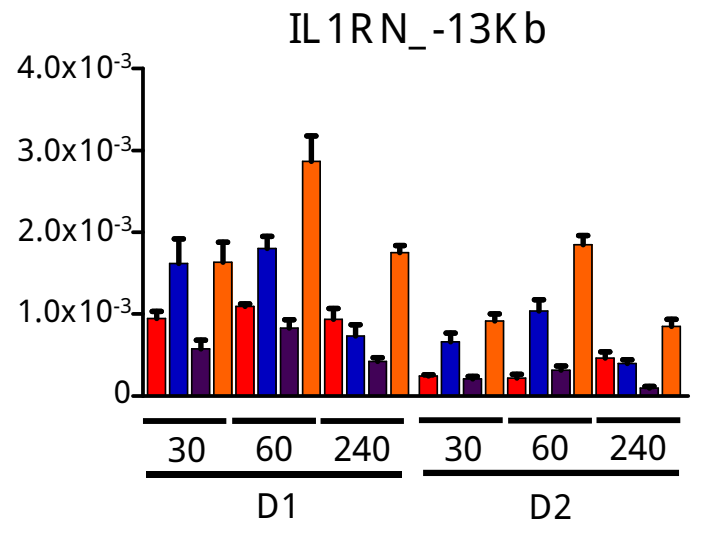

$\square$ veh

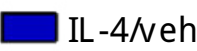

$\square$ L G 268

$\square$ IL-4/L G 268

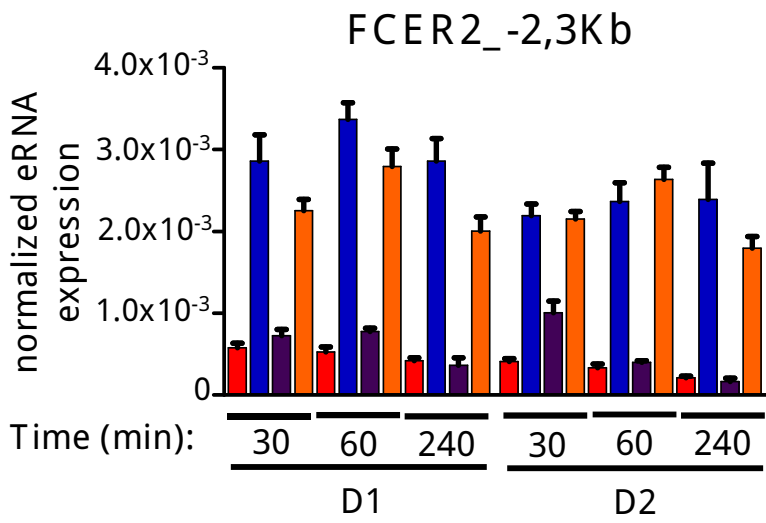

TGM2_-3,3Kb
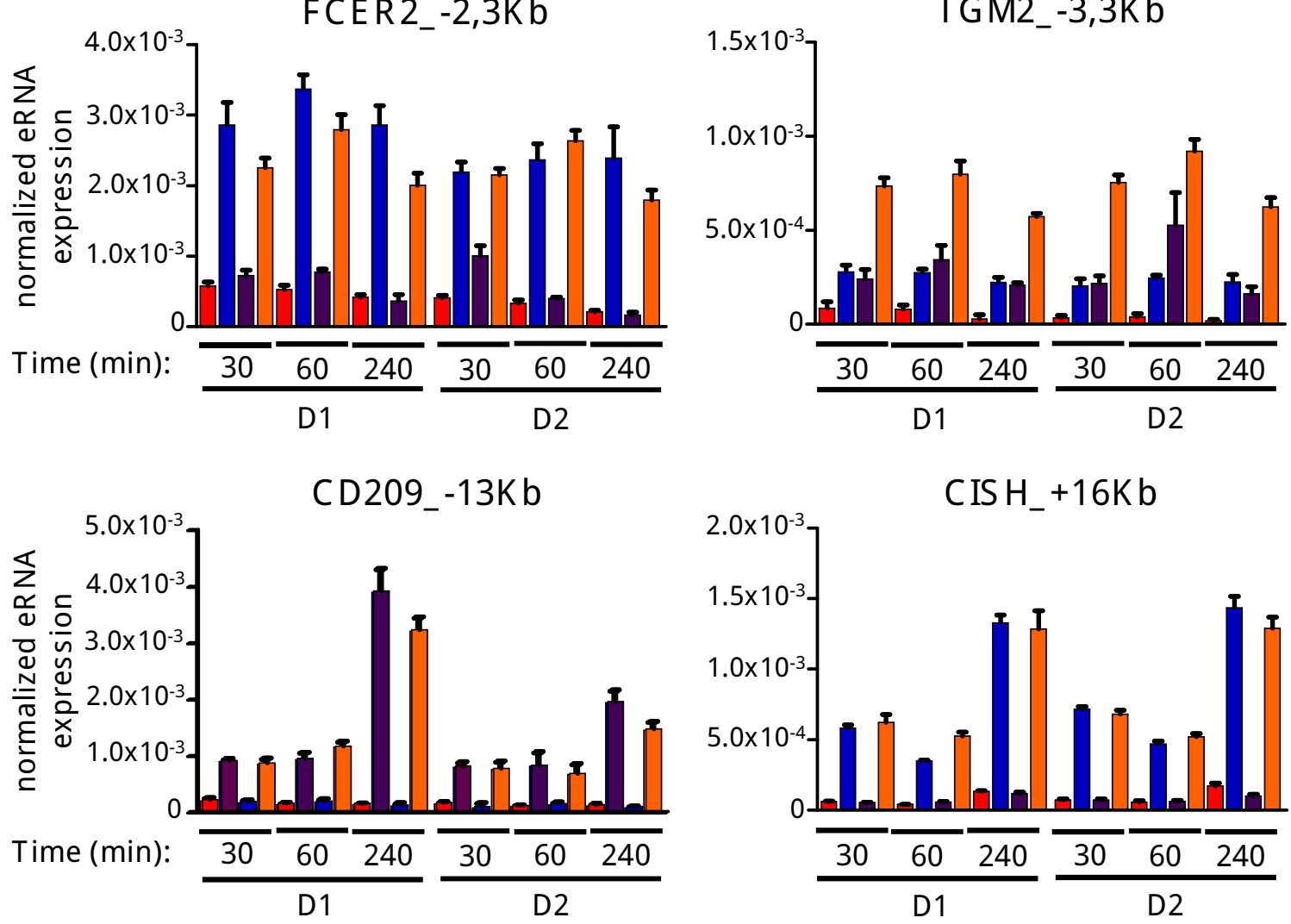
synergistic

potentiating

liganded RXR independent

veh
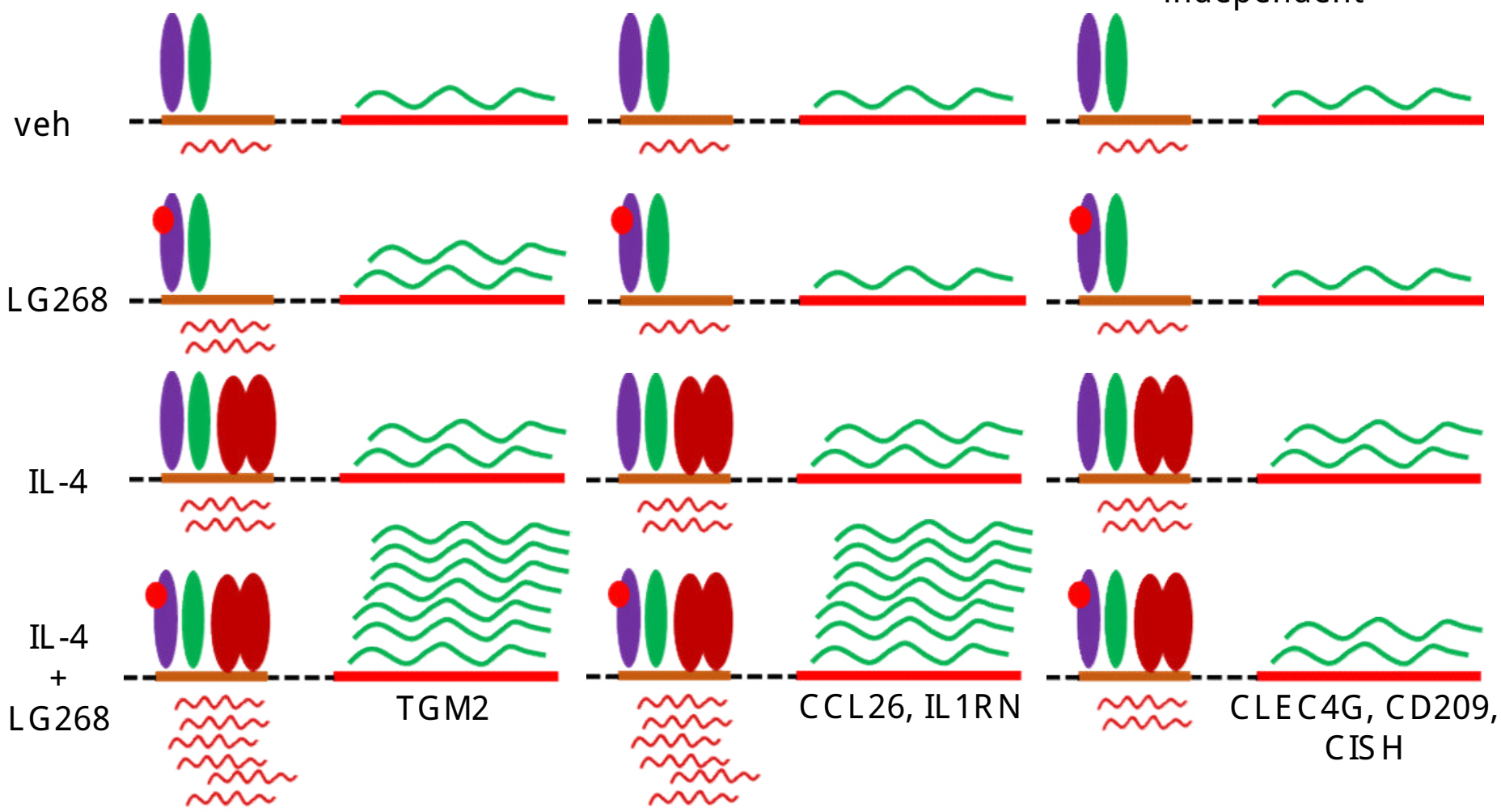

$\sim$ mRNA $\sim$ eRNA RXR NRR STAT6 LG268 
A

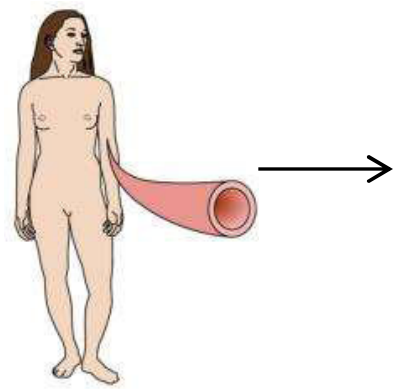

Buffy coat
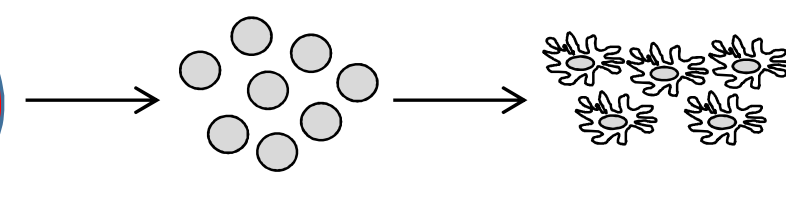

\section{象}

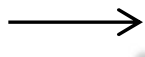

Veh

Magnetic separation of CD14+ monocytes

B
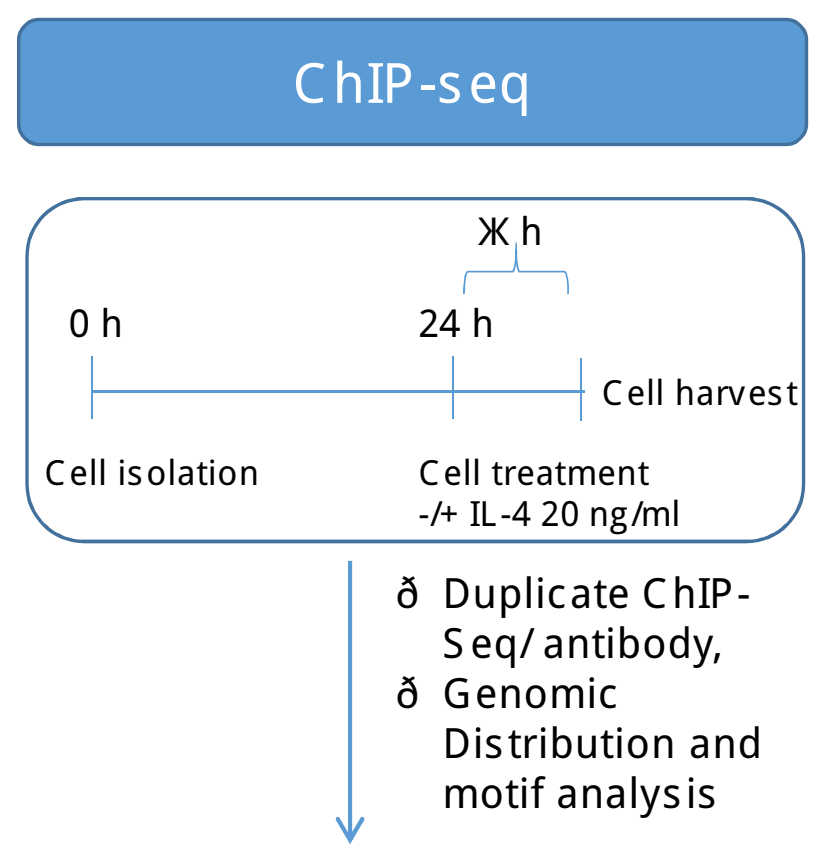

ð human macrophge-specific $\left(\mathrm{H} 3 \mathrm{~K} 27 \mathrm{Ac}+/ \mathrm{PU} .1^{+}\right)$enhancer set

ð STAT6 cistrome

ð RXR cistrome

ð RXR/STAT6 co-peaks
$24 \mathrm{~h}$ attachment on culture dish
Cell treatments

IL -4
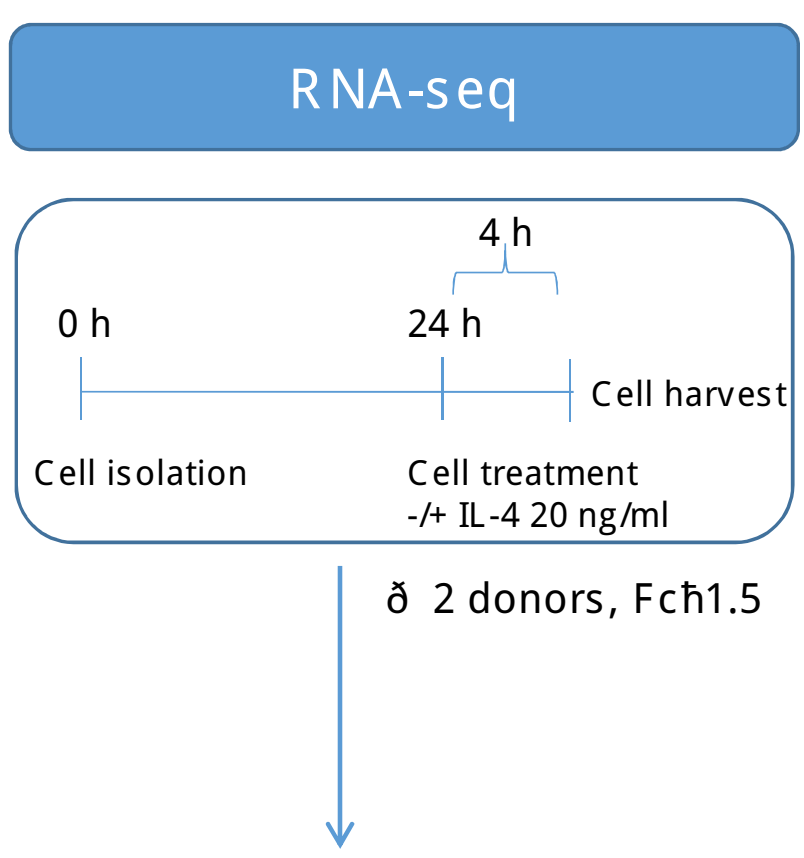
ðL-4-responsive macrophage
transcriptome 
A

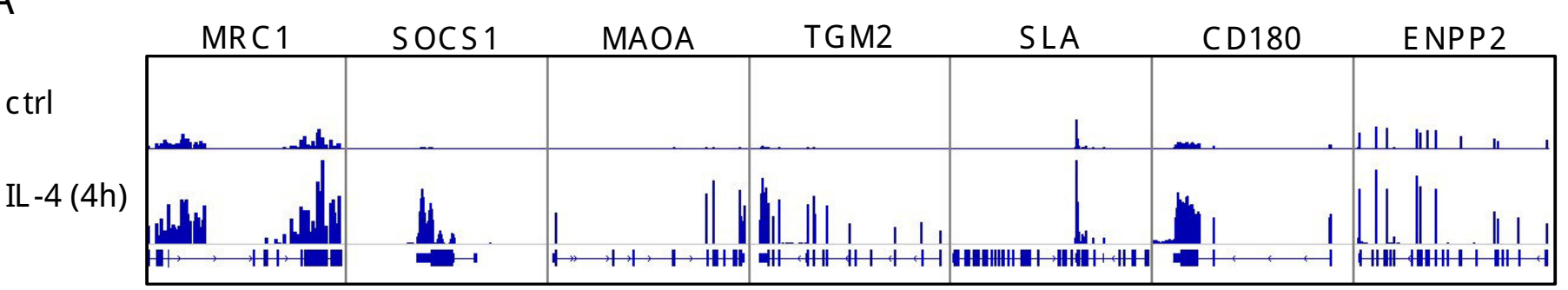

B

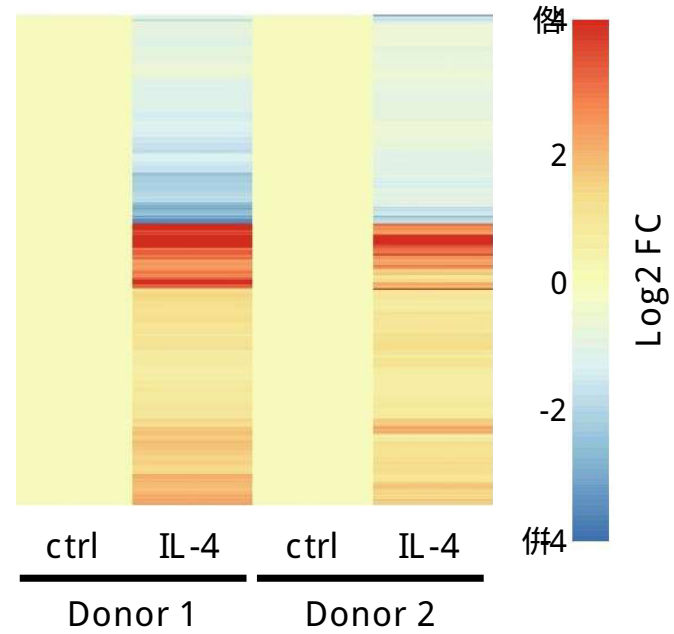

D

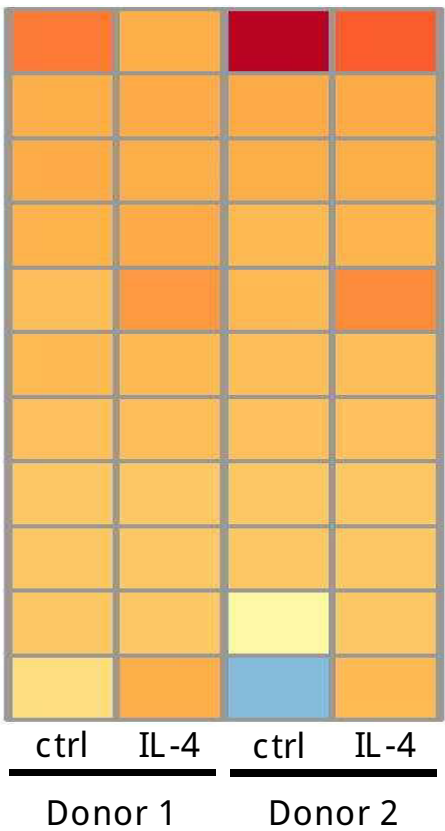

LXR

$\operatorname{LXR} \oint$

$\mathrm{RXR}$

PPAR 200

VDR

RXR $\oint$

RAR 100 주준

THR

PPAR 50

RAR $\therefore$

PPAR $\therefore$

C

\begin{tabular}{l}
1 \\
$\sim$ \\
0 \\
0 \\
\hline
\end{tabular}

Biological process Gene No. p-value FDR

$\begin{array}{cccc}\begin{array}{c}\text { inflammatory response } \\ \text { positive regulation of } \\ \text { inflammatory response } \\ \text { immune response }\end{array} & 56 & 1.27 \mathrm{E}-16 & 2.00 \mathrm{E}-13 \\ \begin{array}{c}\text { cellular response to } \\ \text { interferon-gamma } \\ \text { chemotaxis }\end{array} & 16 & 6.12 \mathrm{E}-11 & 1.11 \mathrm{E}-07 \\ \begin{array}{c}\text { chemokine-mediated } \\ \text { signaling pathway }\end{array} & 16 & 3.41 \mathrm{E}-09 & 6.20 \mathrm{E}-06 \\ \begin{array}{c}\text { response to } \\ \text { lipopolysaccharide } \\ \text { signal transduction }\end{array} & 24 & 1.33 \mathrm{E}-07 & 2.41 \mathrm{E}-04 \\ \text { monocyte chemotaxis } & 12 & 9.89 \mathrm{E}-07 & 4.03 \mathrm{E}-04 \\ \begin{array}{c}\text { lymphocyte chemotaxis } \\ \text { lym }-06\end{array} & 10 & 1.01 \mathrm{E}-06 & 1.83 \mathrm{E}-03\end{array}$


A

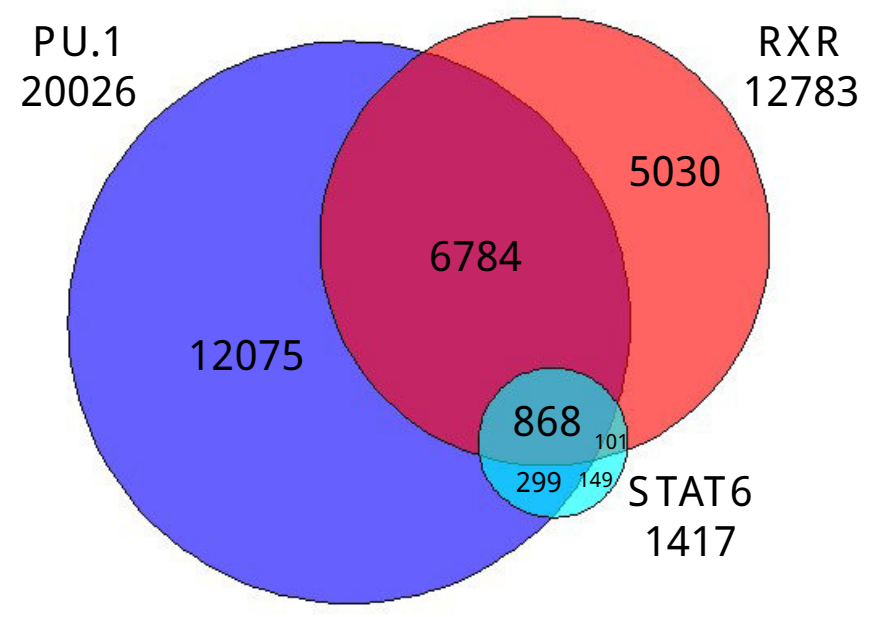

\begin{tabular}{|l|c|l|l|}
\hline Peaks (DiffBind) & PU.1 & RXR & STAT6 \\
\hline Consensus & 26531 & 14125 & 1573 \\
\hline Active (H3K27ac) & 20046 & 12799 & 1417 \\
\hline
\end{tabular}

C

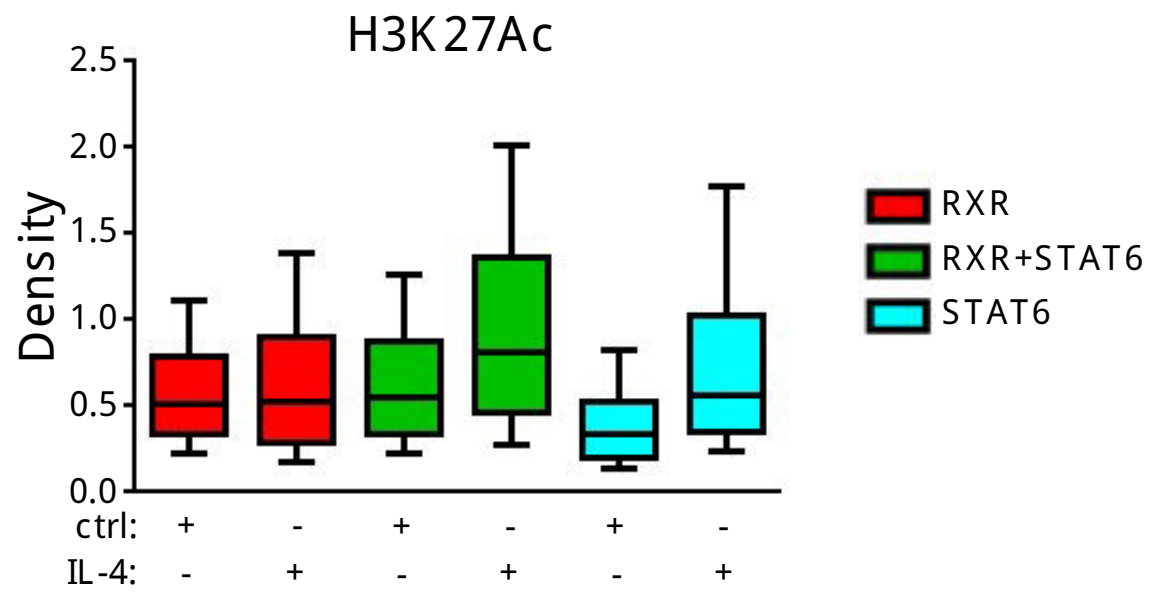

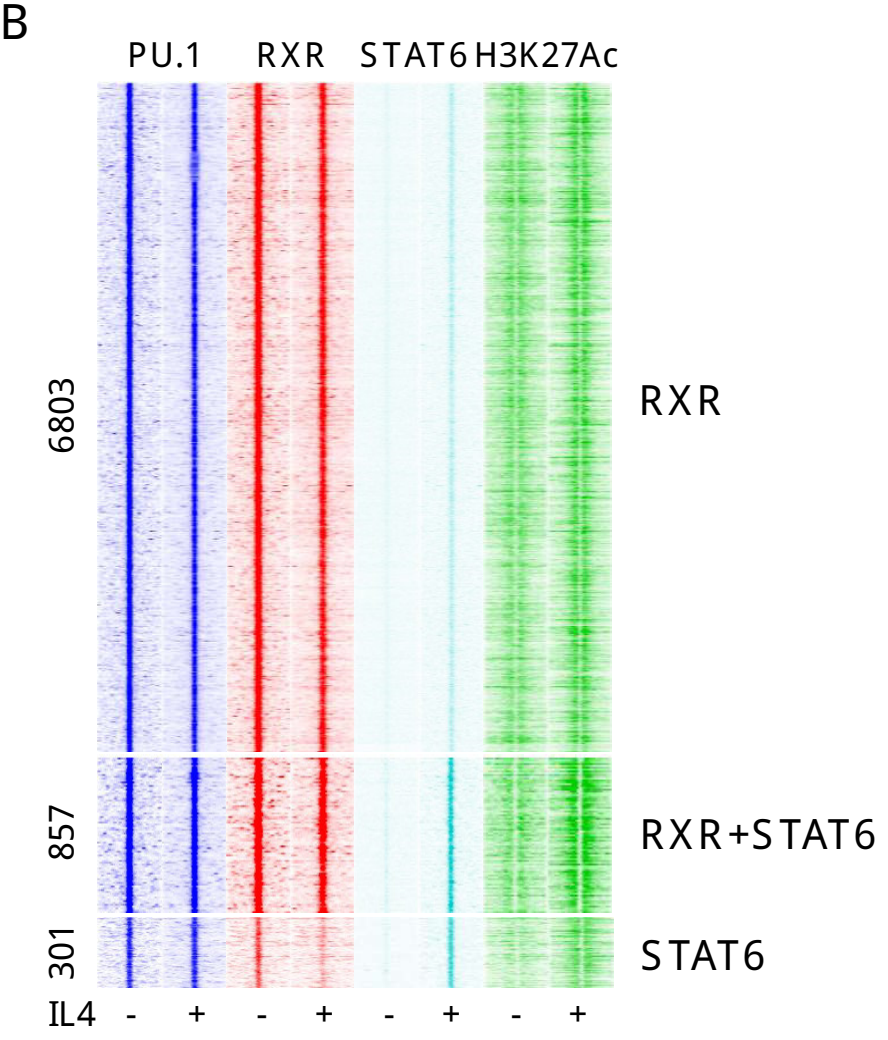


A
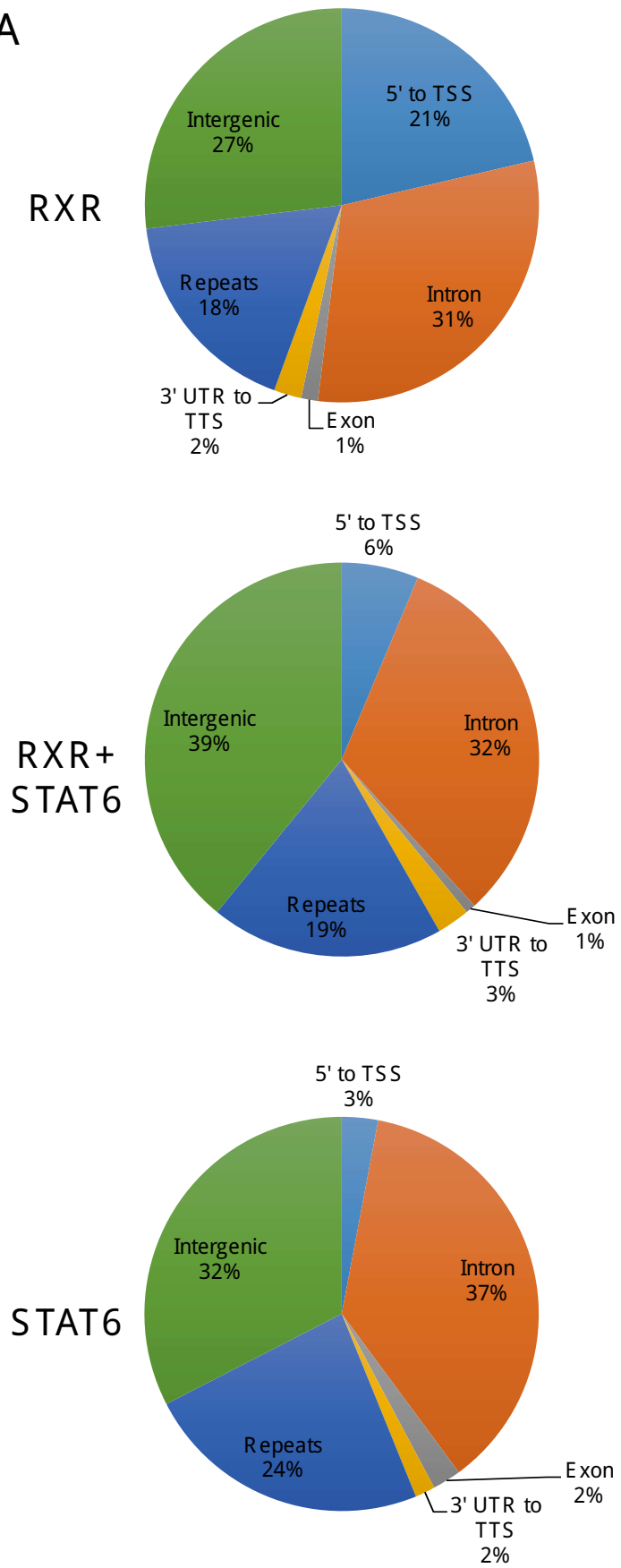

B

\begin{tabular}{|c|c|c|c|c|}
\hline \multicolumn{5}{|c|}{$\begin{array}{l}\text { PU.1 + RXR } \\
(883 / 1000)\end{array}$} \\
\hline & p-value & Target (\%) & $\mathrm{Bg}(\%)$ & Motif \\
\hline$\therefore=G G A A s x$ & $1 \mathrm{E}-278$ & 63.19 & 12.12 & PU.1 \\
\hline$T_{x} x_{x} \mathrm{CAA}$ & $1 \mathrm{E}-75$ & 16.53 & 2.31 & C/EBP \\
\hline$G G T T A C T=E G G T C A=$ & $1 \mathrm{E}-71$ & 2.94 & 0 & DR4 \\
\hline$T_{\subseteq A_{\varepsilon}} T C A$ & $1 \mathrm{E}-62$ & 20.84 & 4.78 & AP-1 \\
\hline$=\pi G A G_{C} T C A$ & $1 \mathrm{E}-41$ & 27.75 & 11.09 & NR half \\
\hline \multicolumn{5}{|c|}{$\begin{array}{c}\text { PU.1 + RXR + STAT 6 } \\
(703 / 857)\end{array}$} \\
\hline & p-value & Target (\%) & $\mathrm{Bg}(\%)$ & Motif \\
\hline$\ldots G G A A_{-T}$ & $1 \mathrm{E}-247$ & 52.54 & 6.21 & PU.1 \\
\hline $\mathrm{TT}_{\mathrm{a}}^{\mathrm{a} A}$ & $1 E-124$ & 36.76 & 6.31 & C/EBP \\
\hline TTc $=\mathrm{GAA}$ & $1 \mathrm{E}-92$ & 36.48 & 8.63 & STAT6 \\
\hline$=$ TGAeTCA & $1 E-38$ & 20.99 & 6.2 & AP-1 \\
\hline$G G G_{A \cap T T T} T C_{c}$ & $1 E-26$ & 21.27 & 8.28 & REL \\
\hline$\Rightarrow A A C C A C$ & $1 \mathrm{E}-22$ & 10.99 & 2.86 & RUNX \\
\hline \multicolumn{5}{|c|}{$\begin{array}{l}\text { PU.1 + STAT } 6 \\
(233 / 301)\end{array}$} \\
\hline & p-value & Target (\%) & $\mathrm{Bg}(\%)$ & Motif \\
\hline$G G A A_{\cong} T$ & $1 \mathrm{E}-80$ & 61.8 & 10.25 & PU.1 \\
\hline$-T=C_{z}=G A A_{-}$ & $1 \mathrm{E}-57$ & 45.06 & 6.96 & STAT6 \\
\hline${ }_{\triangle} T_{\mathrm{sT}} \mathrm{G}_{s} \mathrm{AA} I$ & $1 \mathrm{E}-22$ & 28.76 & 7.12 & $C / E B P$ \\
\hline a TGA ICA $=$ & $1 \mathrm{E}-18$ & 16.74 & 2.72 & $A P-1$ \\
\hline
\end{tabular}


A

IL -4 responsive genes-associated

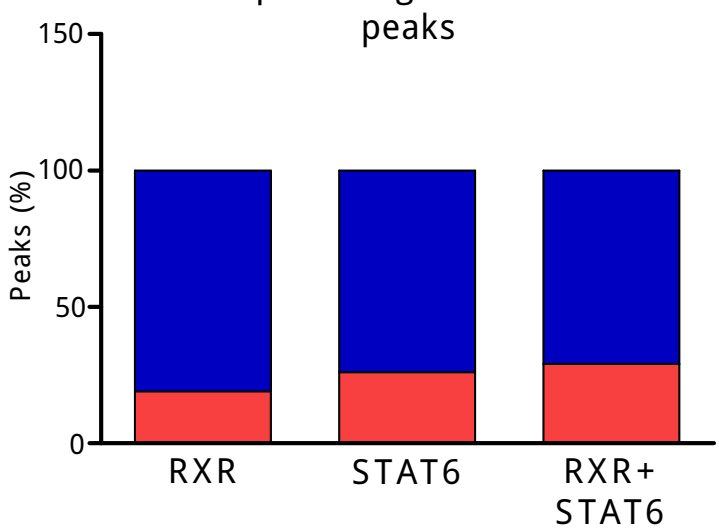

B

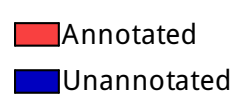

Peak distribution relative to TSS

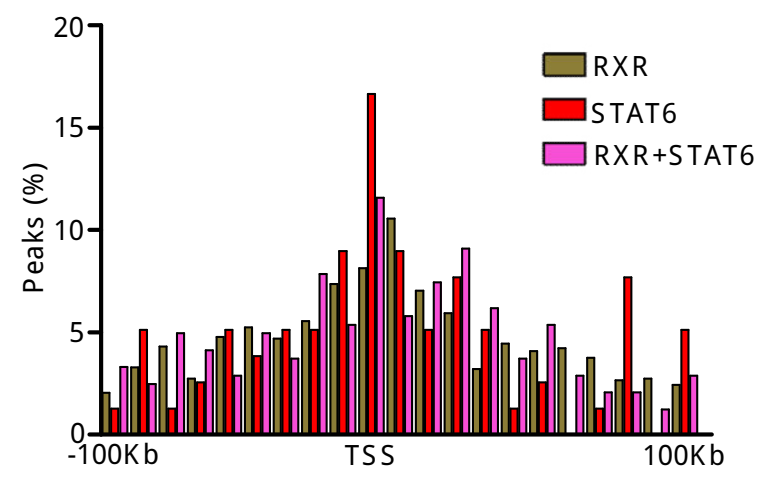

C

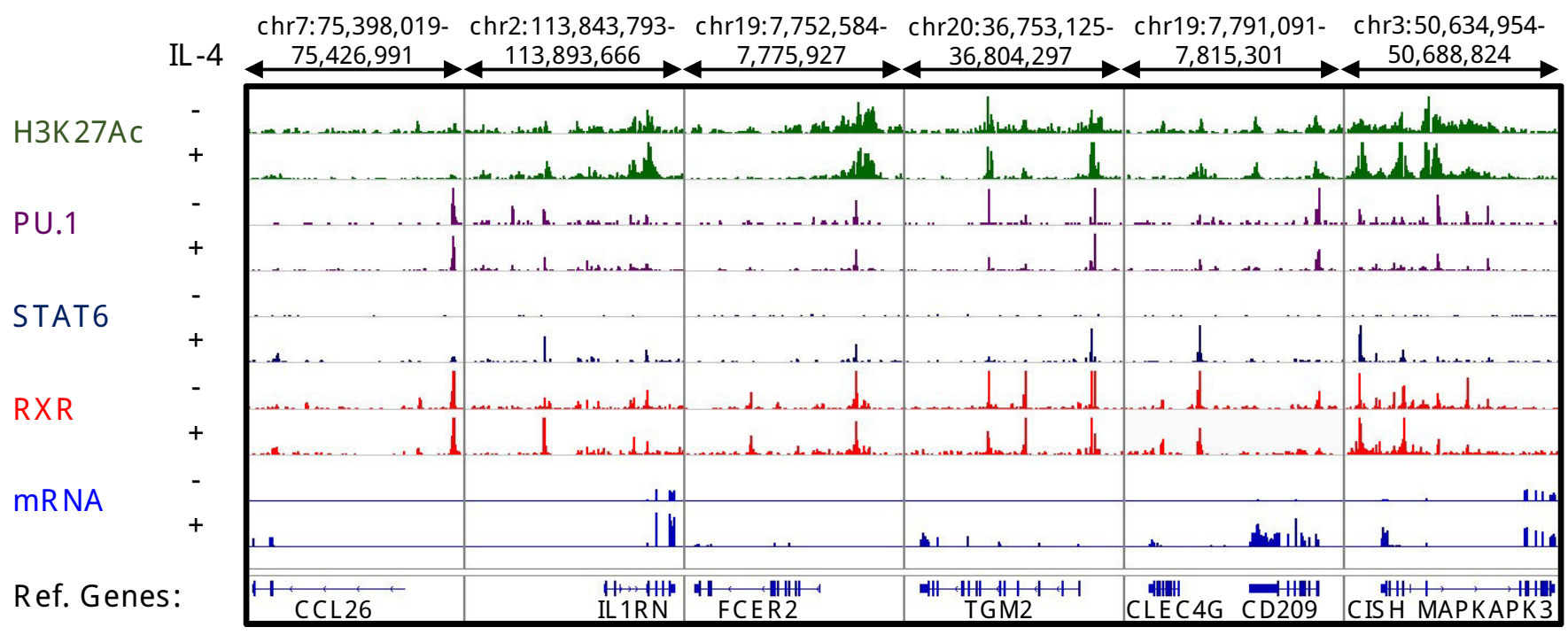


A

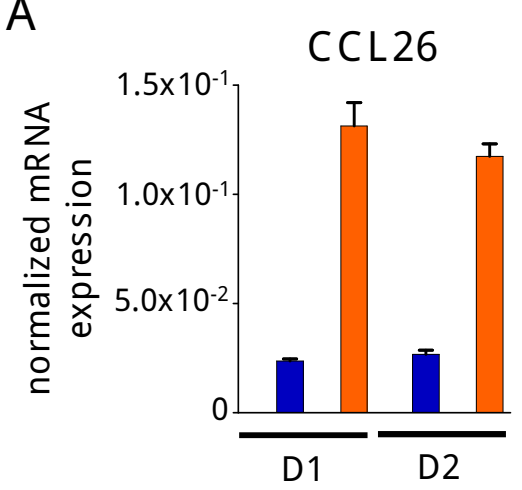

IL 1 R N

FCER2 TGM2
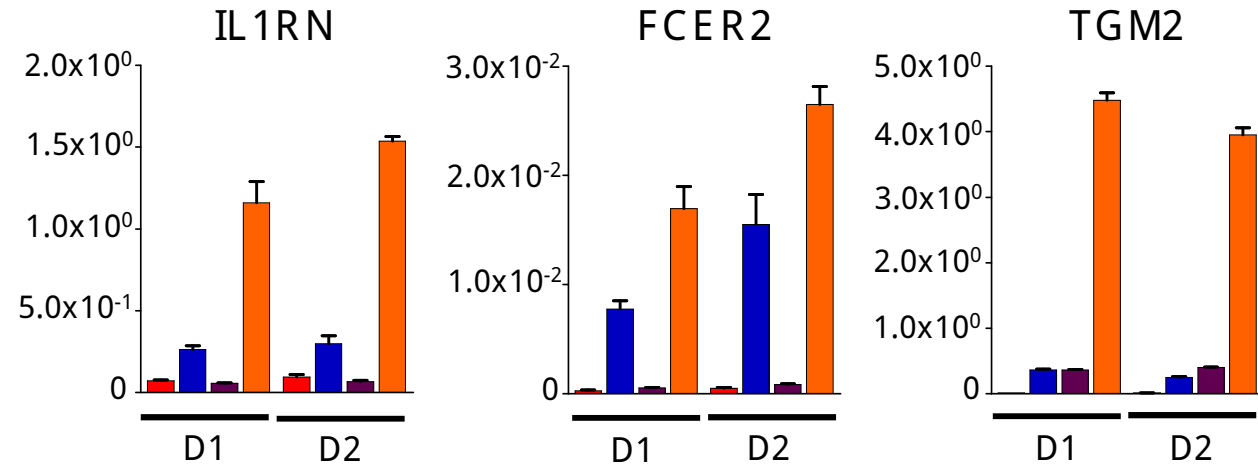

CLEC4G

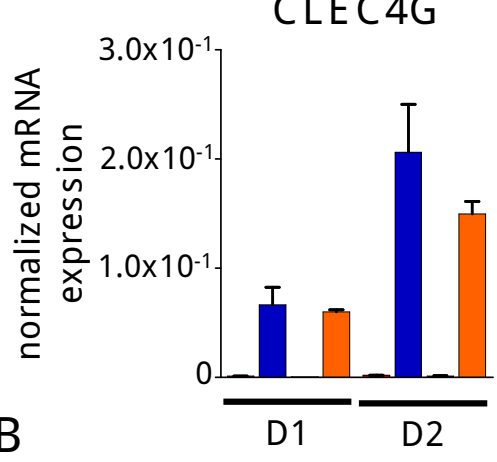

CD209
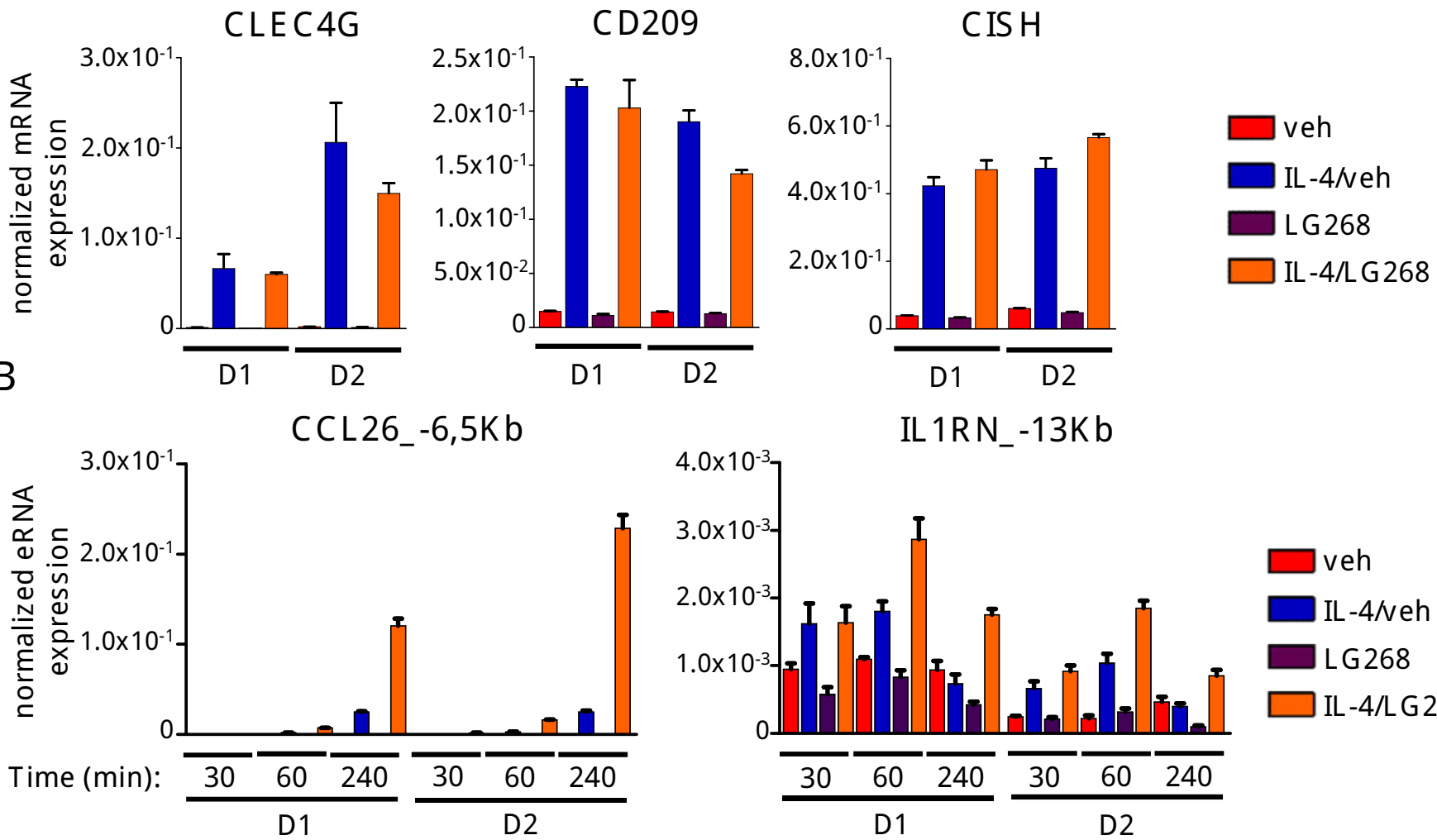

IL $1 R N_{-}-13 K b$

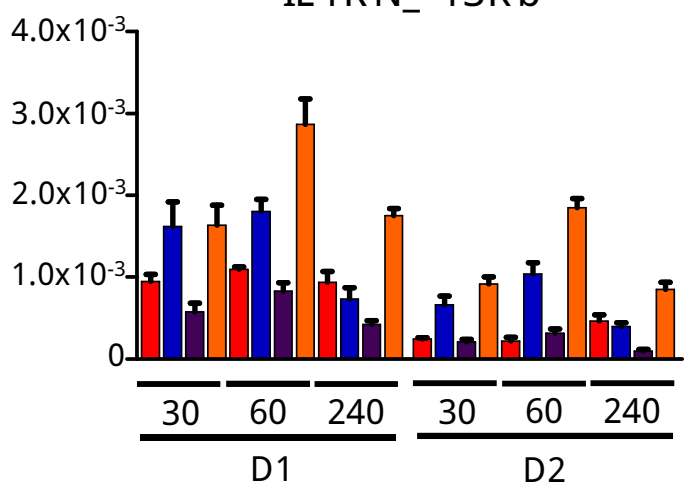

$\square$ veh

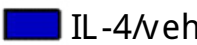

$\square$ LG268

$\square$ IL -4/L G 268

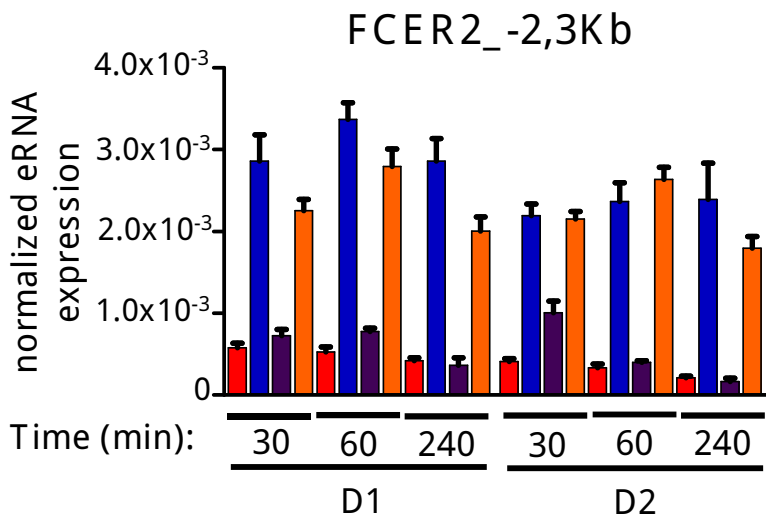

TGM2_-3,3Kb
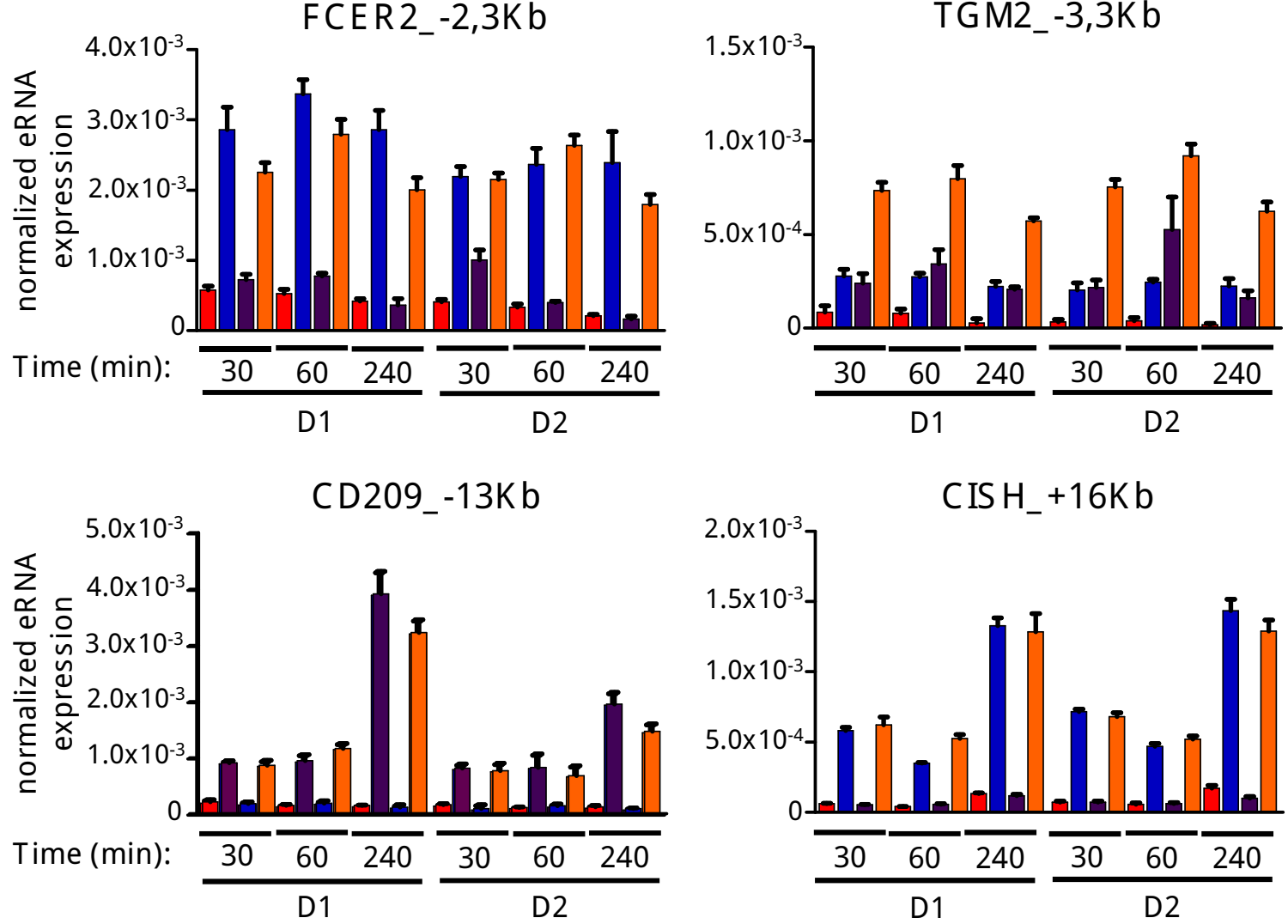
synergistic

potentiating

liganded RXR independent

veh
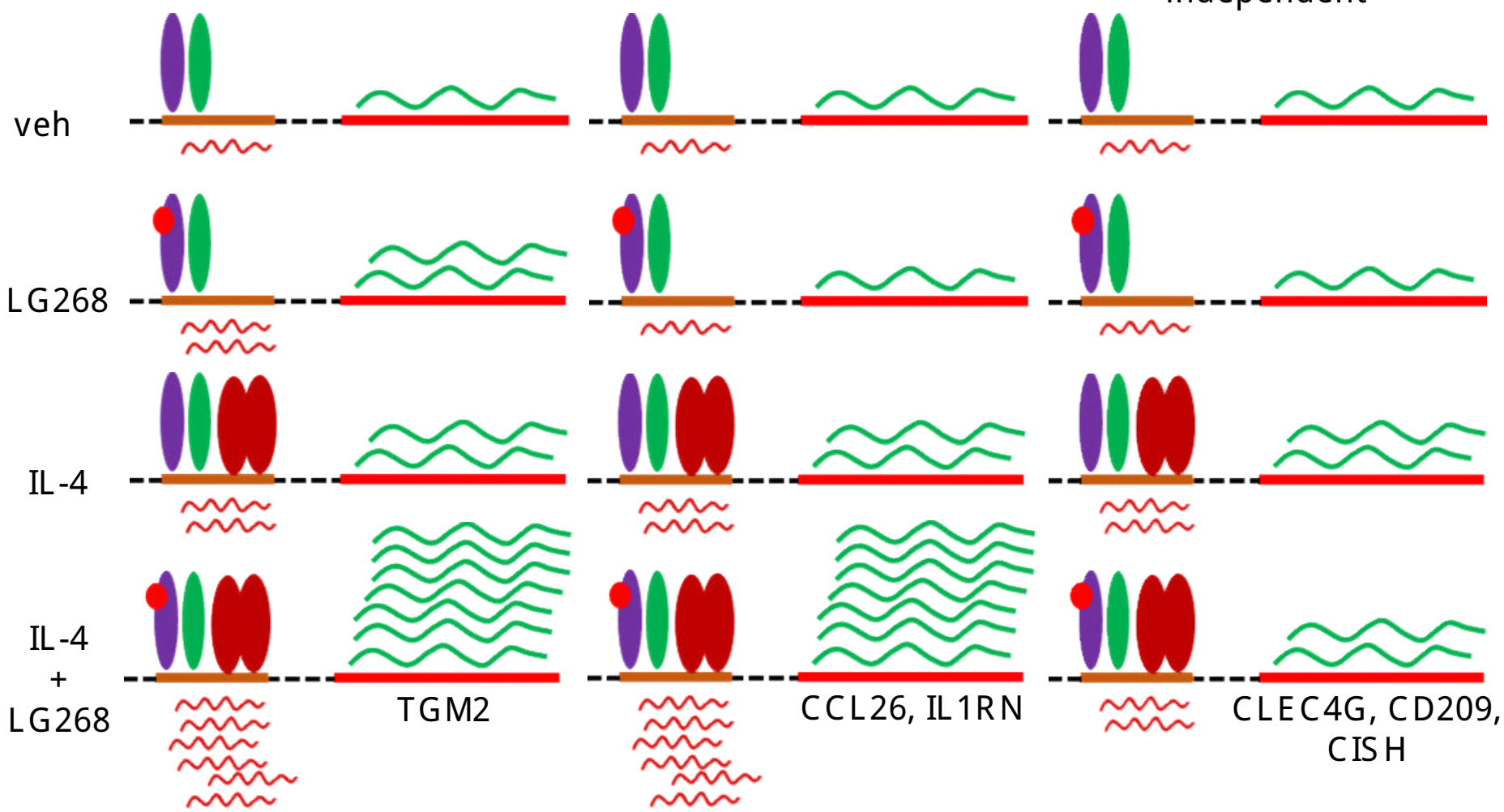

$\sim$ mRNA $\sim$ eRNA RXR NRR STAT6 LG268 
A

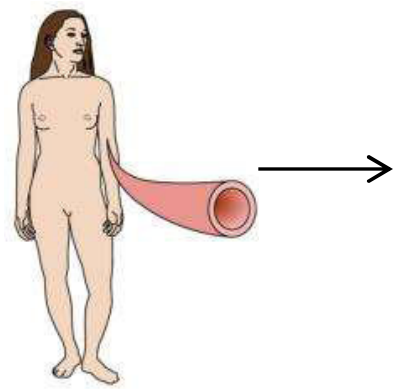

Buffy coat
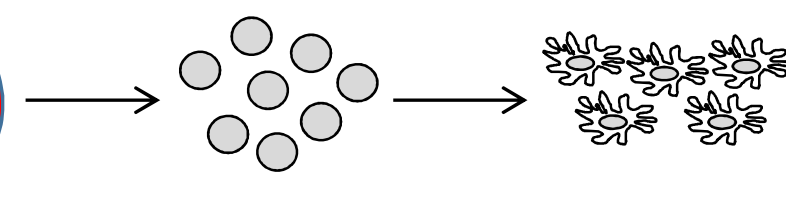

\section{象}

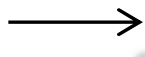

Veh

Magnetic separation of CD14+ monocytes

B
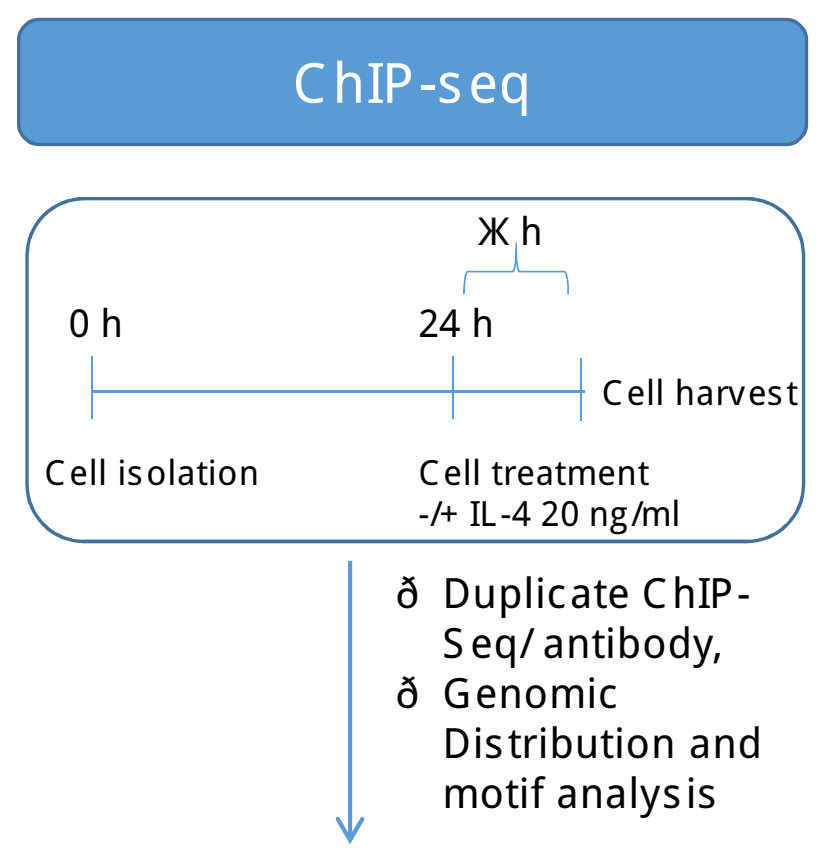

ð human macrophge-specific $\left(\mathrm{H} 3 \mathrm{~K} 27 \mathrm{Ac}+/ \mathrm{PU} .1^{+}\right)$enhancer set

ð STAT6 cistrome

ð RXR cistrome

ð RXR/STAT6 co-peaks
$24 \mathrm{~h}$ attachment on culture dish
Cell treatments

IL -4
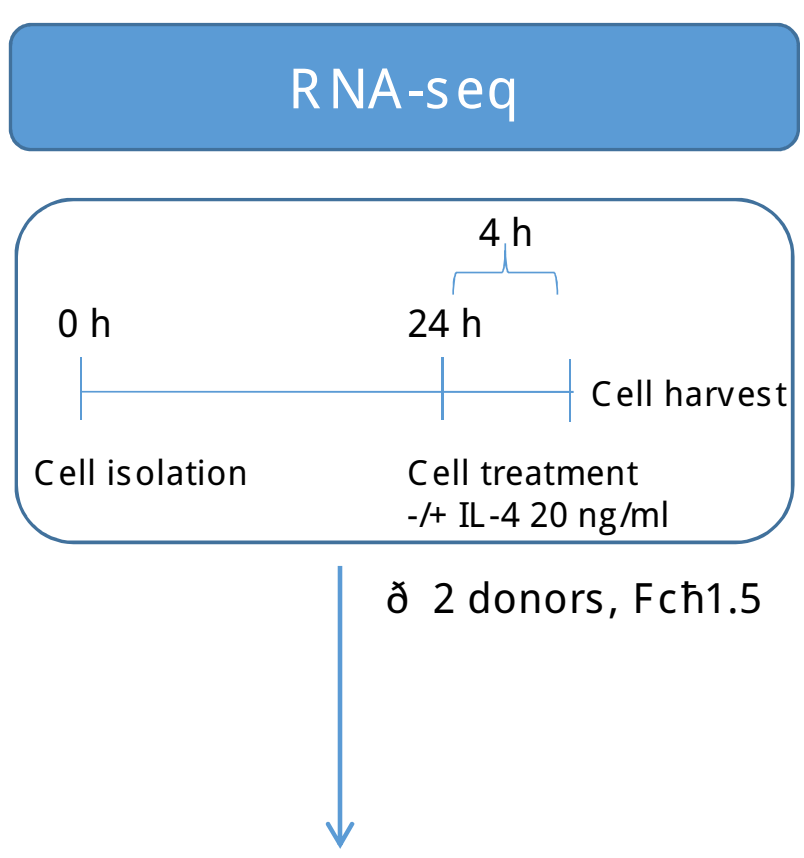
ðL-4-responsive macrophage
transcriptome 
A

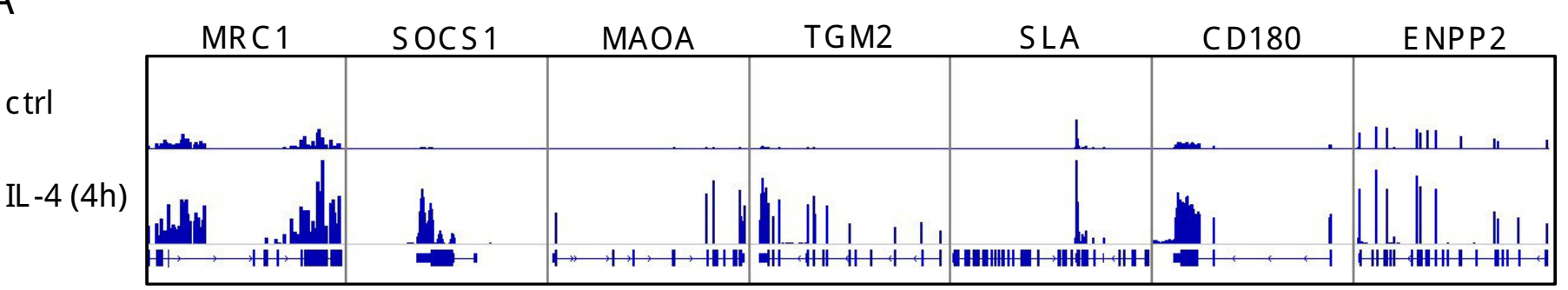

B

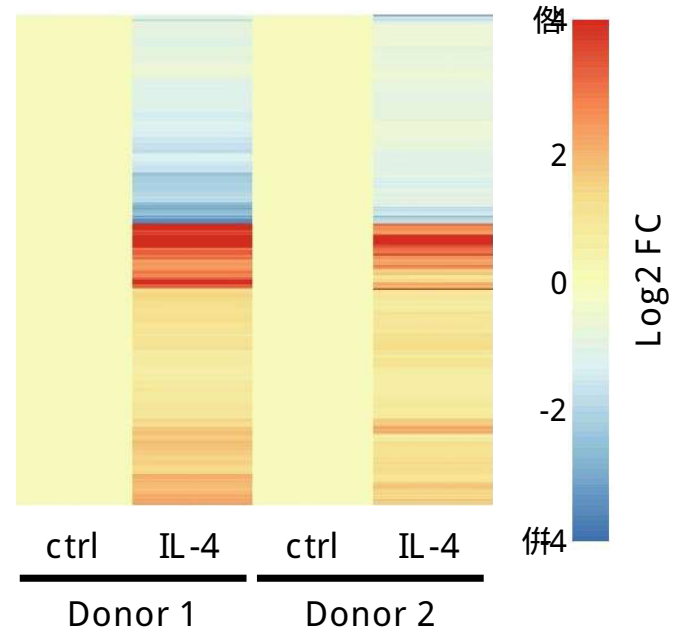

D

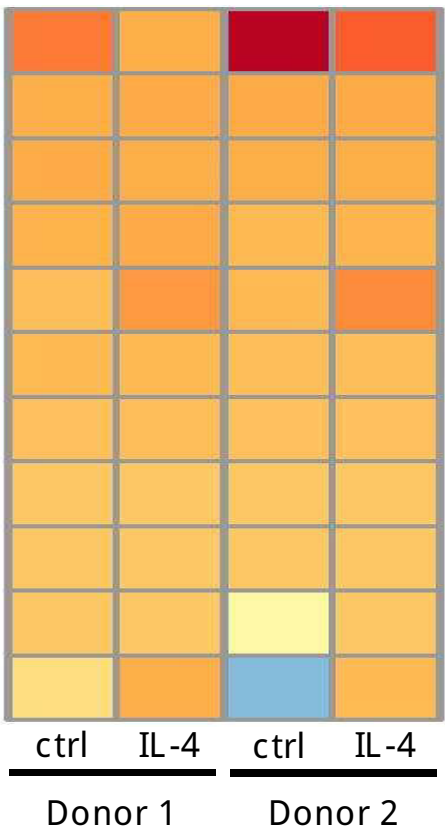

LXR

$\operatorname{LXR} \oint$

$\mathrm{RXR}$

PPAR 200

VDR

RXR $\oint$

RAR 100 주준

THR

PPAR 50

RAR $\therefore$

PPAR $\therefore$

C

\begin{tabular}{l}
1 \\
$\sim$ \\
0 \\
0 \\
\hline
\end{tabular}

Biological process Gene No. p-value FDR

$\begin{array}{cccc}\begin{array}{c}\text { inflammatory response } \\ \text { positive regulation of } \\ \text { inflammatory response } \\ \text { immune response }\end{array} & 56 & 1.27 \mathrm{E}-16 & 2.00 \mathrm{E}-13 \\ \begin{array}{c}\text { cellular response to } \\ \text { interferon-gamma } \\ \text { chemotaxis }\end{array} & 16 & 6.12 \mathrm{E}-11 & 1.11 \mathrm{E}-07 \\ \begin{array}{c}\text { chemokine-mediated } \\ \text { signaling pathway }\end{array} & 16 & 3.41 \mathrm{E}-09 & 6.20 \mathrm{E}-06 \\ \begin{array}{c}\text { response to } \\ \text { lipopolysaccharide } \\ \text { signal transduction }\end{array} & 24 & 1.33 \mathrm{E}-07 & 2.41 \mathrm{E}-04 \\ \text { monocyte chemotaxis } & 12 & 9.89 \mathrm{E}-07 & 4.03 \mathrm{E}-04 \\ \begin{array}{c}\text { lymphocyte chemotaxis } \\ \text { lym }-06\end{array} & 10 & 1.01 \mathrm{E}-06 & 1.83 \mathrm{E}-03\end{array}$


A

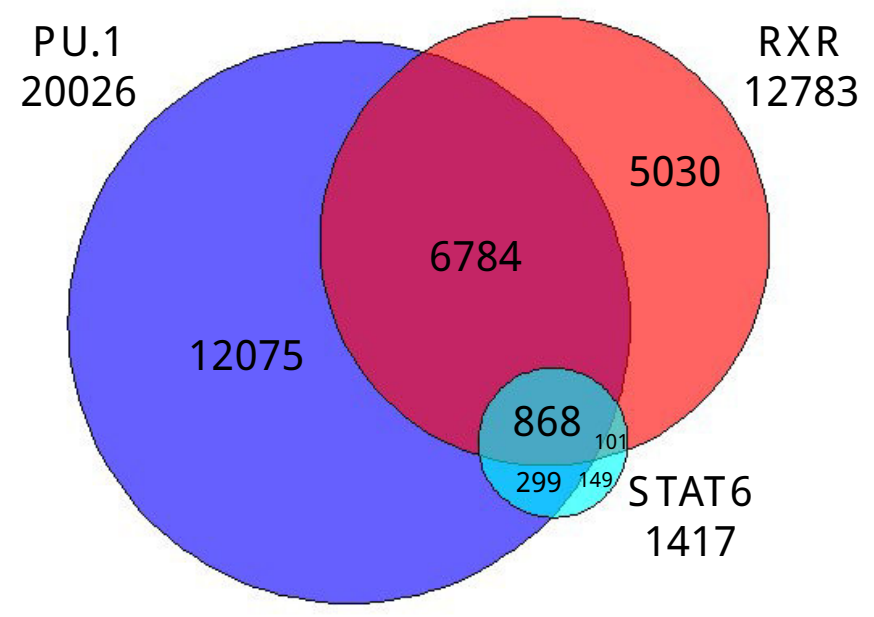

\begin{tabular}{|l|c|l|l|}
\hline Peaks (DiffBind) & PU.1 & RXR & STAT6 \\
\hline Consensus & 26531 & 14125 & 1573 \\
\hline Active (H3K27ac) & 20046 & 12799 & 1417 \\
\hline
\end{tabular}

C

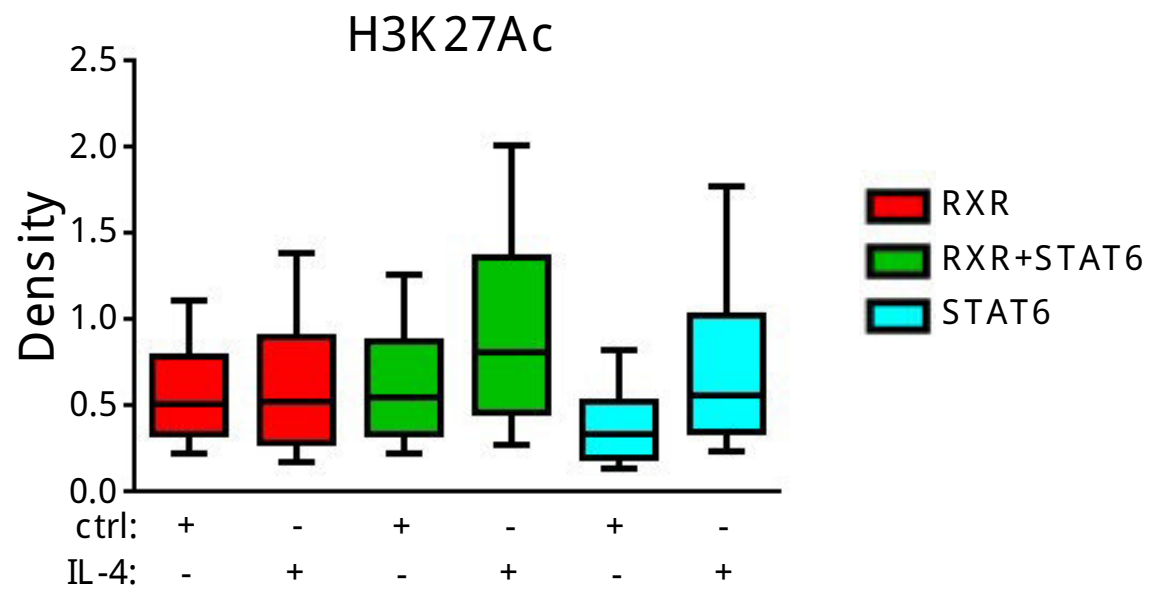

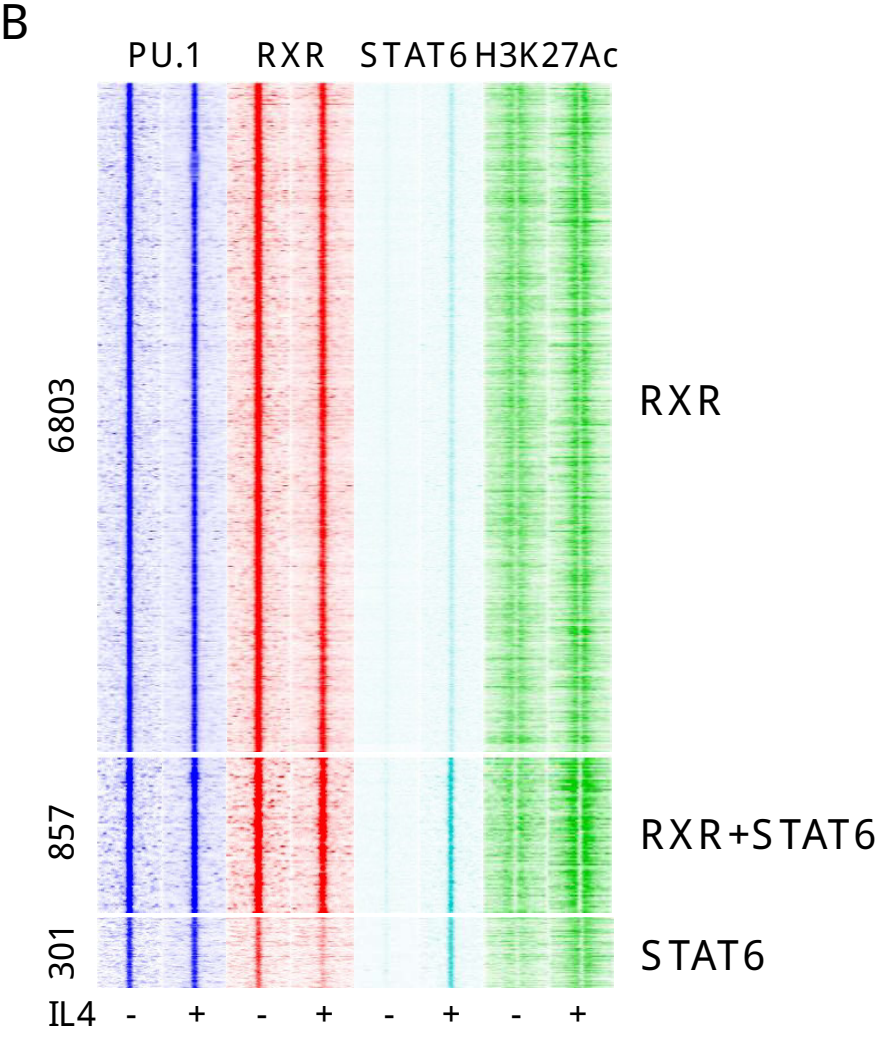


A
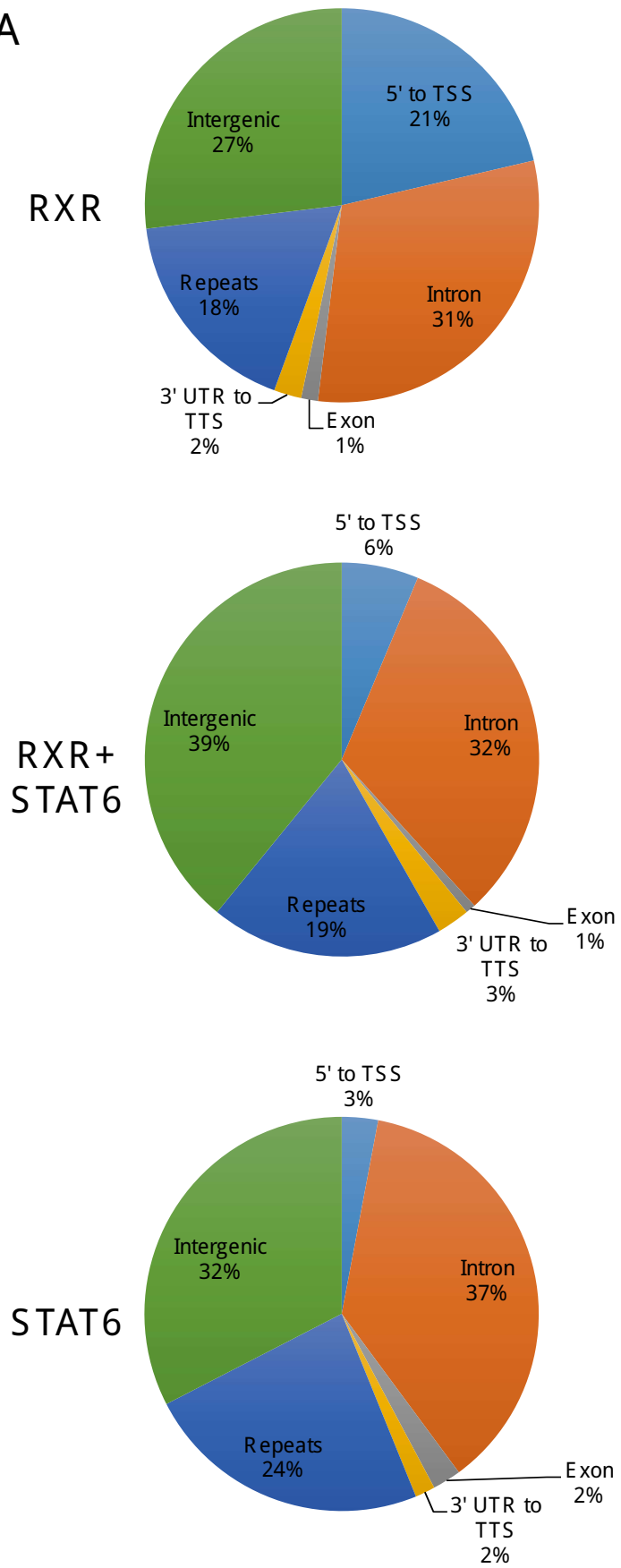

B

\begin{tabular}{|c|c|c|c|c|}
\hline \multicolumn{5}{|c|}{$\begin{array}{l}\text { PU.1 + RXR } \\
(883 / 1000)\end{array}$} \\
\hline & p-value & Target (\%) & $\mathrm{Bg}(\%)$ & Motif \\
\hline$\therefore=G G A A s x$ & $1 \mathrm{E}-278$ & 63.19 & 12.12 & PU.1 \\
\hline$T_{x} x_{x} \mathrm{CAA}$ & $1 \mathrm{E}-75$ & 16.53 & 2.31 & C/EBP \\
\hline$G G T T A C T=E G G T C A=$ & $1 \mathrm{E}-71$ & 2.94 & 0 & DR4 \\
\hline$T_{\subseteq A_{\varepsilon}} T C A$ & $1 \mathrm{E}-62$ & 20.84 & 4.78 & AP-1 \\
\hline$=\pi G A G_{C} T C A$ & $1 \mathrm{E}-41$ & 27.75 & 11.09 & NR half \\
\hline \multicolumn{5}{|c|}{$\begin{array}{c}\text { PU.1 + RXR + STAT 6 } \\
(703 / 857)\end{array}$} \\
\hline & p-value & Target (\%) & $\mathrm{Bg}(\%)$ & Motif \\
\hline$\ldots G G A A_{-T}$ & $1 \mathrm{E}-247$ & 52.54 & 6.21 & PU.1 \\
\hline $\mathrm{TT}_{\mathrm{a}}^{\mathrm{a} A}$ & $1 E-124$ & 36.76 & 6.31 & C/EBP \\
\hline TTc $=\mathrm{GAA}$ & $1 \mathrm{E}-92$ & 36.48 & 8.63 & STAT6 \\
\hline$=$ TGAeTCA & $1 E-38$ & 20.99 & 6.2 & AP-1 \\
\hline$G G G_{A \cap T T T} T C_{c}$ & $1 E-26$ & 21.27 & 8.28 & REL \\
\hline$\Rightarrow A A C C A C$ & $1 \mathrm{E}-22$ & 10.99 & 2.86 & RUNX \\
\hline \multicolumn{5}{|c|}{$\begin{array}{l}\text { PU.1 + STAT } 6 \\
(233 / 301)\end{array}$} \\
\hline & p-value & Target (\%) & $\mathrm{Bg}(\%)$ & Motif \\
\hline$G G A A_{\cong} T$ & $1 \mathrm{E}-80$ & 61.8 & 10.25 & PU.1 \\
\hline$-T=C_{z}=G A A_{-}$ & $1 \mathrm{E}-57$ & 45.06 & 6.96 & STAT6 \\
\hline${ }_{\triangle} T_{\mathrm{sT}} \mathrm{G}_{s} \mathrm{AA} I$ & $1 \mathrm{E}-22$ & 28.76 & 7.12 & $C / E B P$ \\
\hline a TGA ICA $=$ & $1 \mathrm{E}-18$ & 16.74 & 2.72 & $A P-1$ \\
\hline
\end{tabular}


A

IL -4 responsive genes-associated

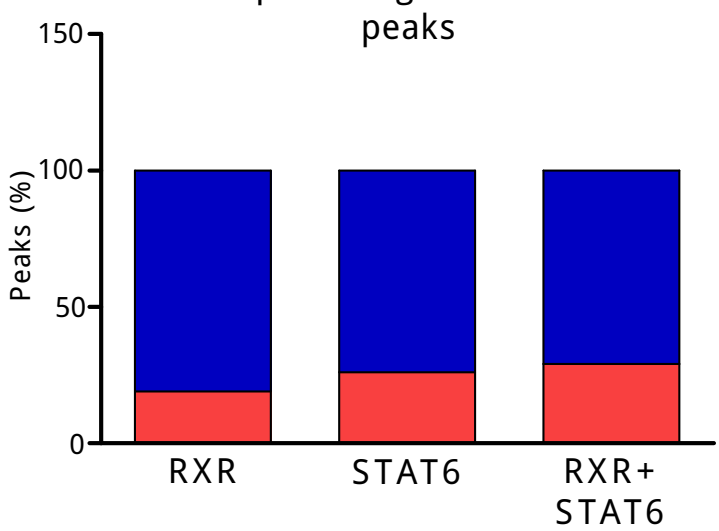

B

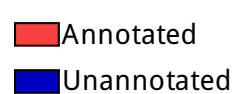

Peak distribution relative to TSS

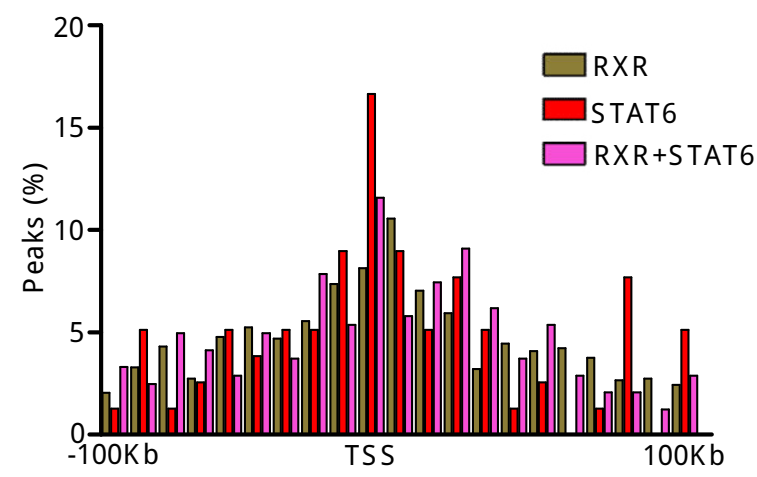

C

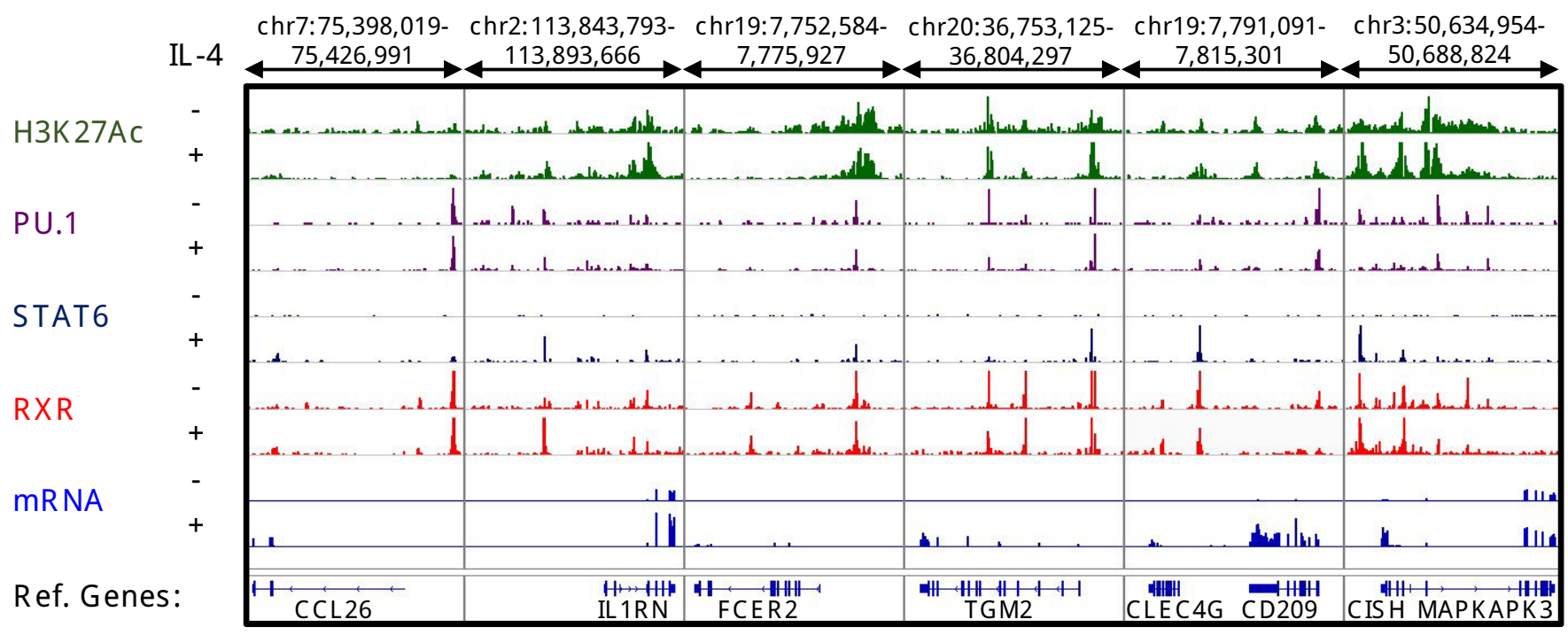


A

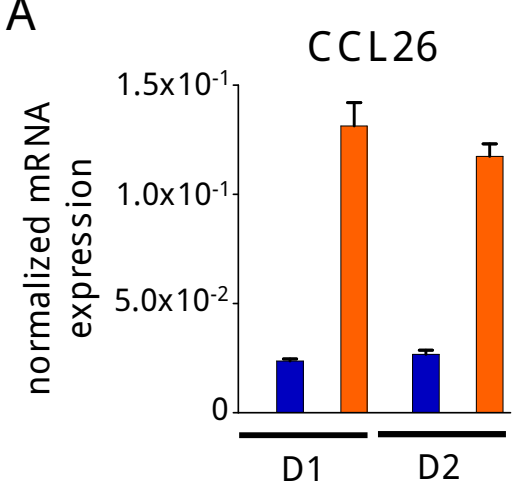

IL 1 R N

FCER2 TGM2
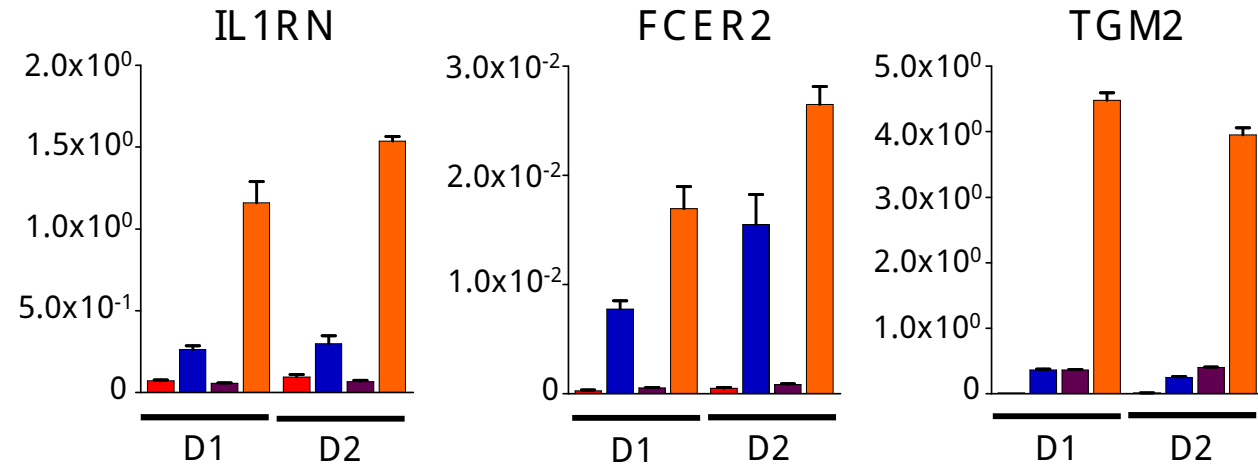

CLEC4G

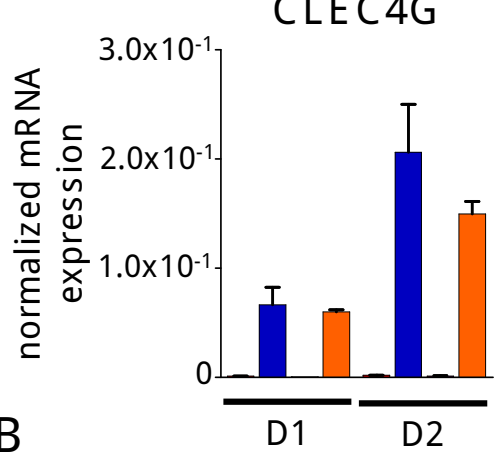

CD209
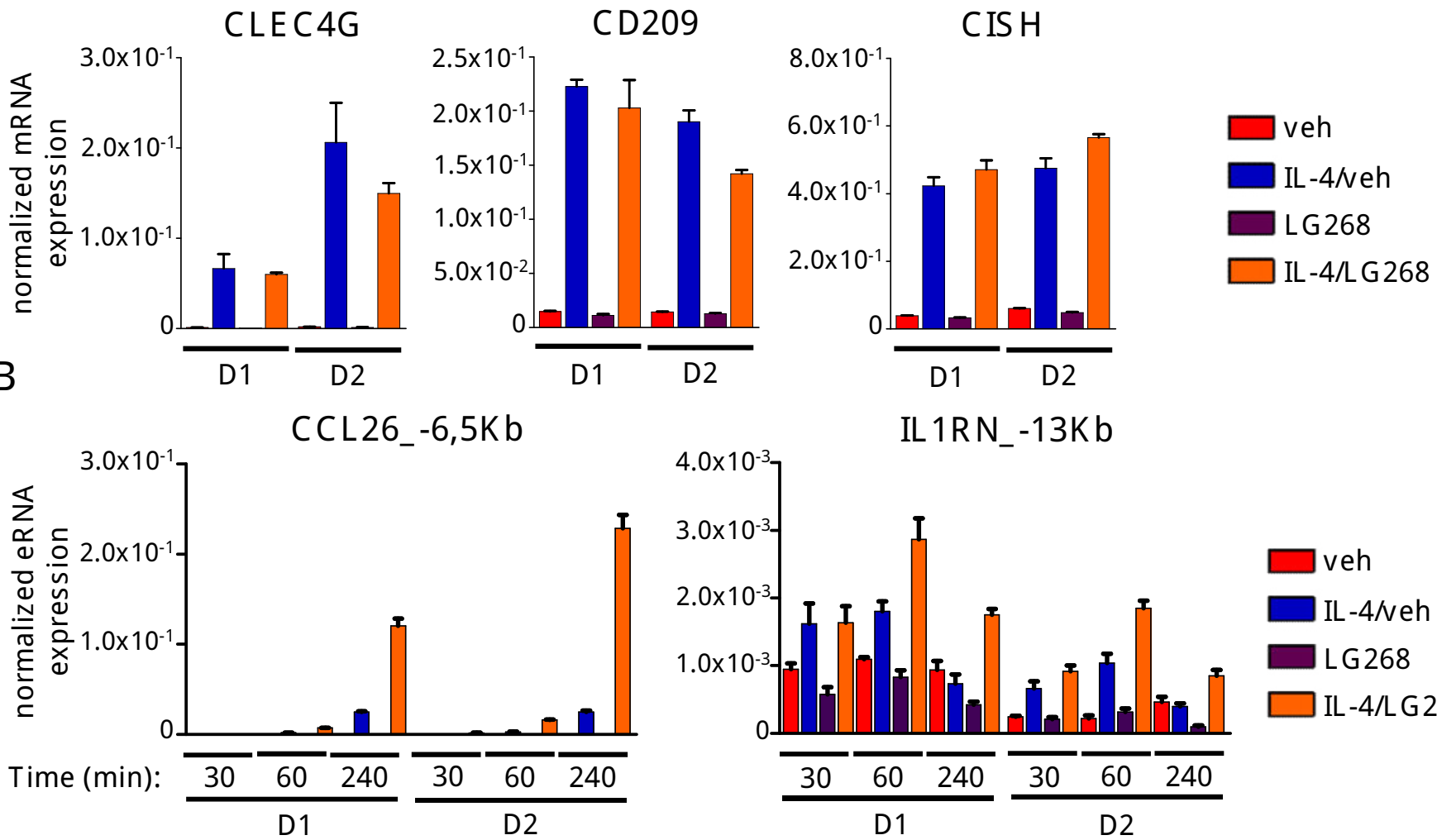

IL $1 R N_{-}-13 K b$

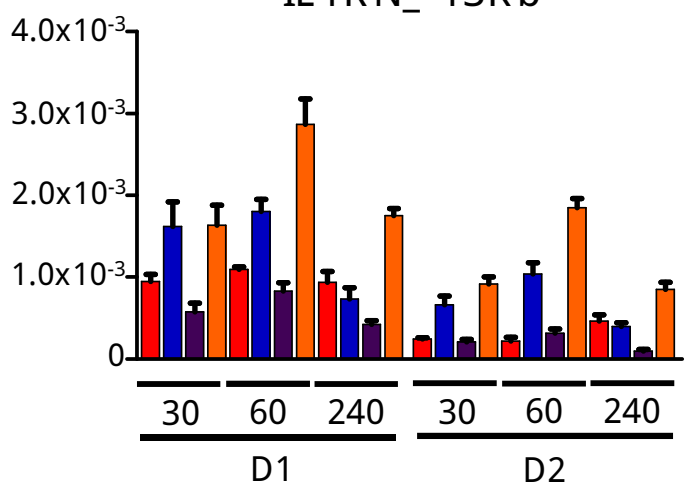

$\square$ veh

IL-4/veh

$\square$ LG268

$\square$ IL -4/L G 268

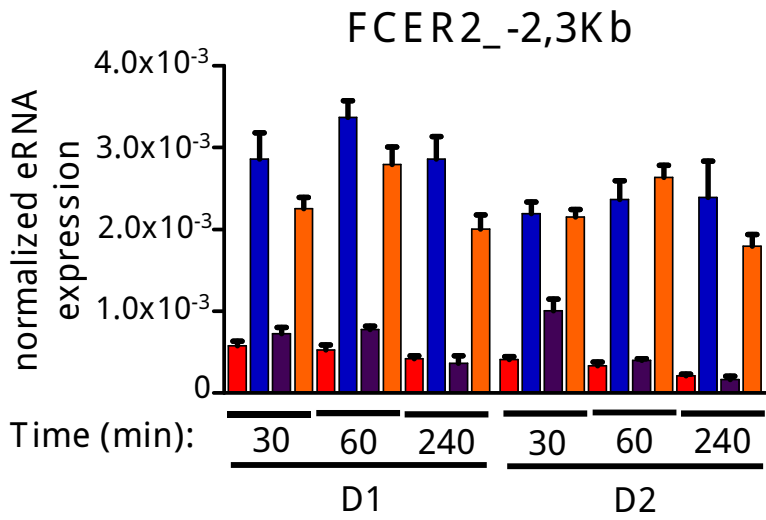

TGM2_-3,3Kb
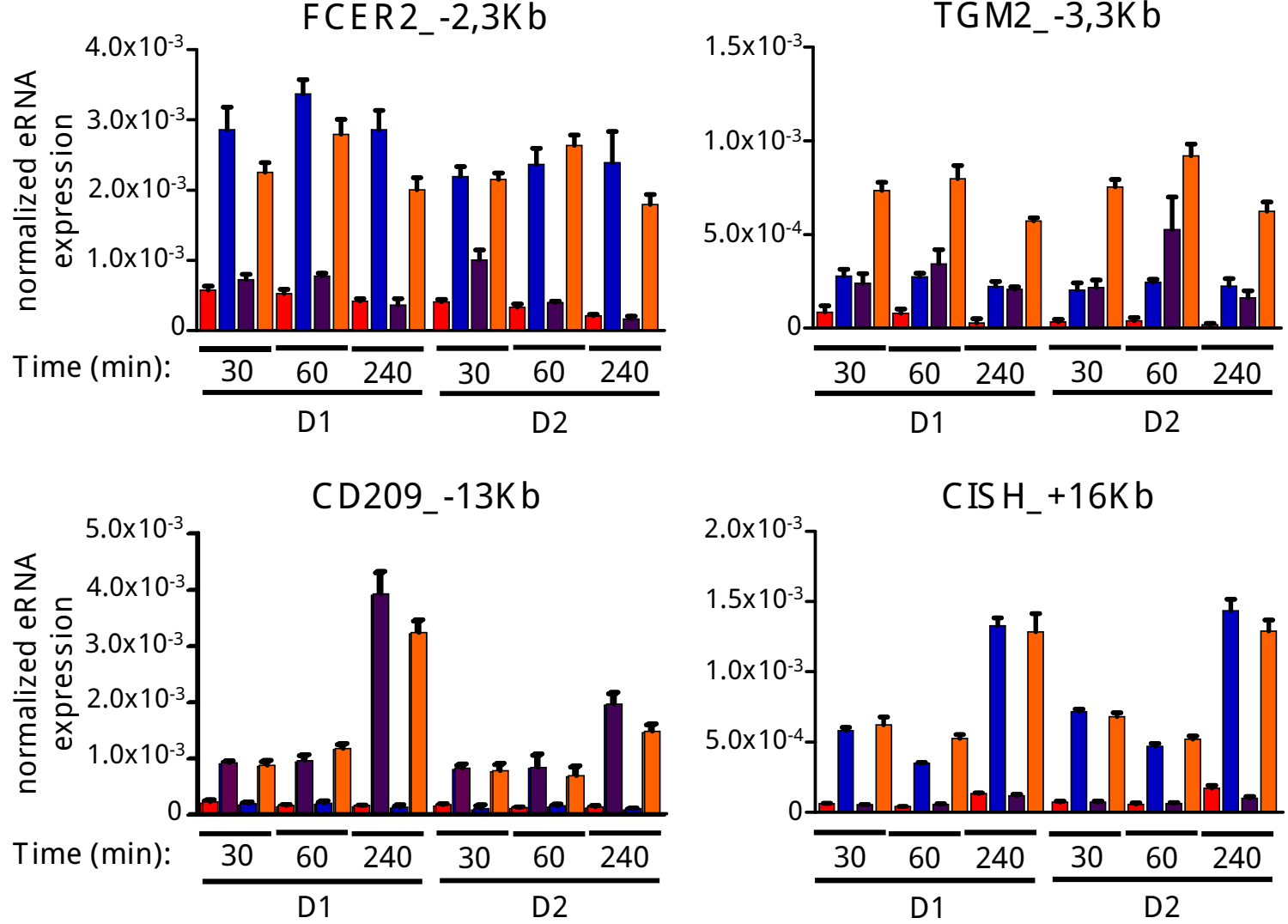
synergistic

potentiating

liganded RXR independent

veh
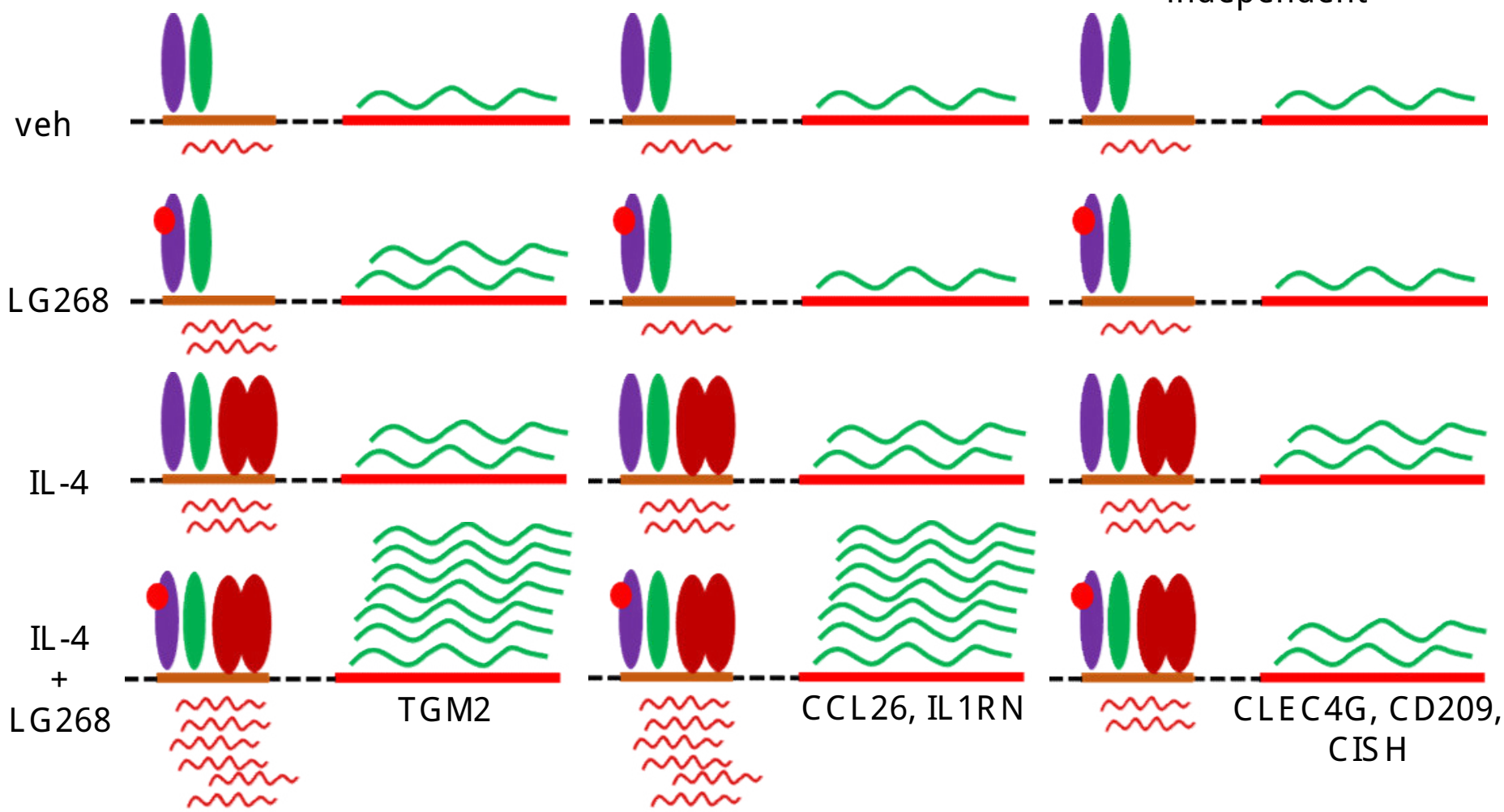

$\sim$ mRNA $\sim$ eRNA RXR NRR STAT6 LG268 
A

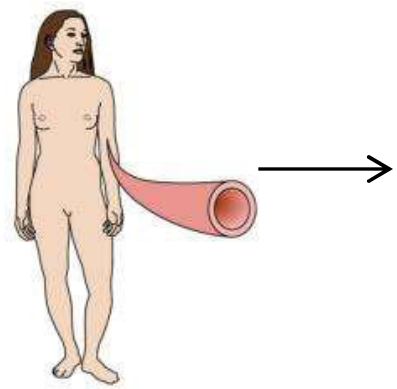

Buffy coat
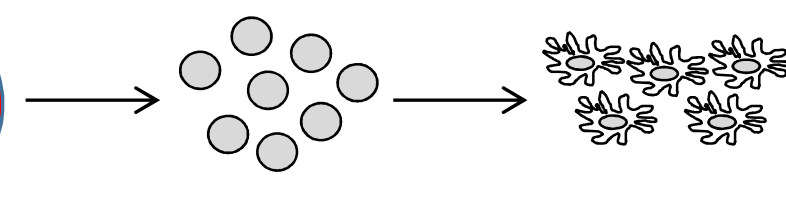

\section{象}

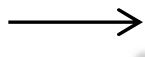

Veh

Magnetic separation of CD14+ monocytes

B
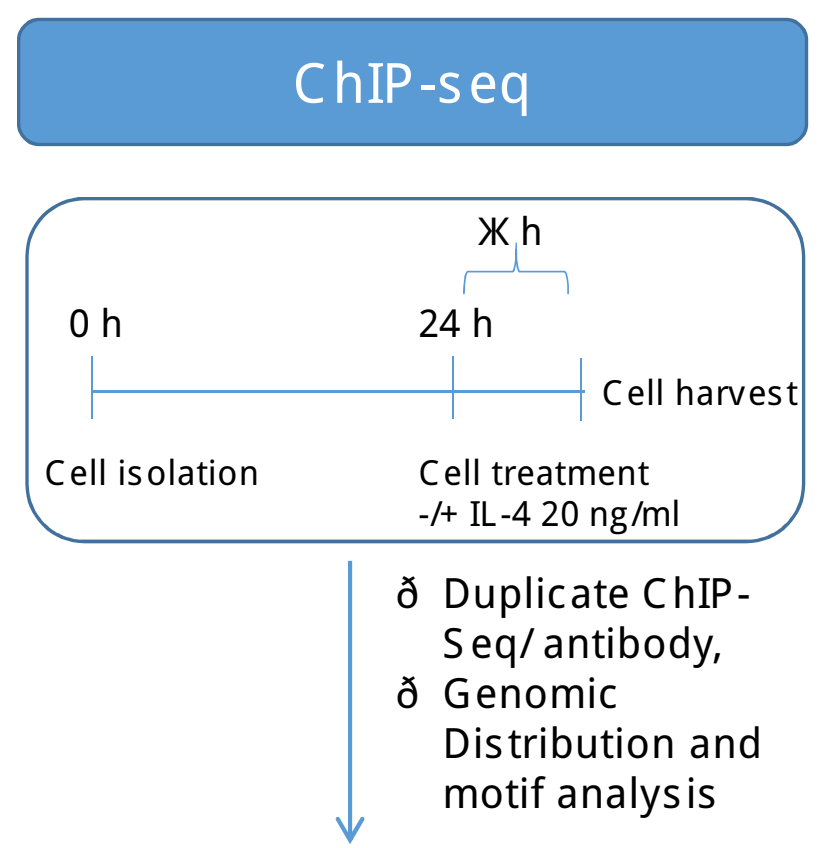

ð human macrophge-specific $\left(\mathrm{H} 3 \mathrm{~K} 27 \mathrm{Ac}+/ \mathrm{PU} .1^{+}\right)$enhancer set

ð STAT6 cistrome

ð RXR cistrome

ð RXR/STAT6 co-peaks
$24 \mathrm{~h}$ attachment on culture dish
Cell treatments

IL -4
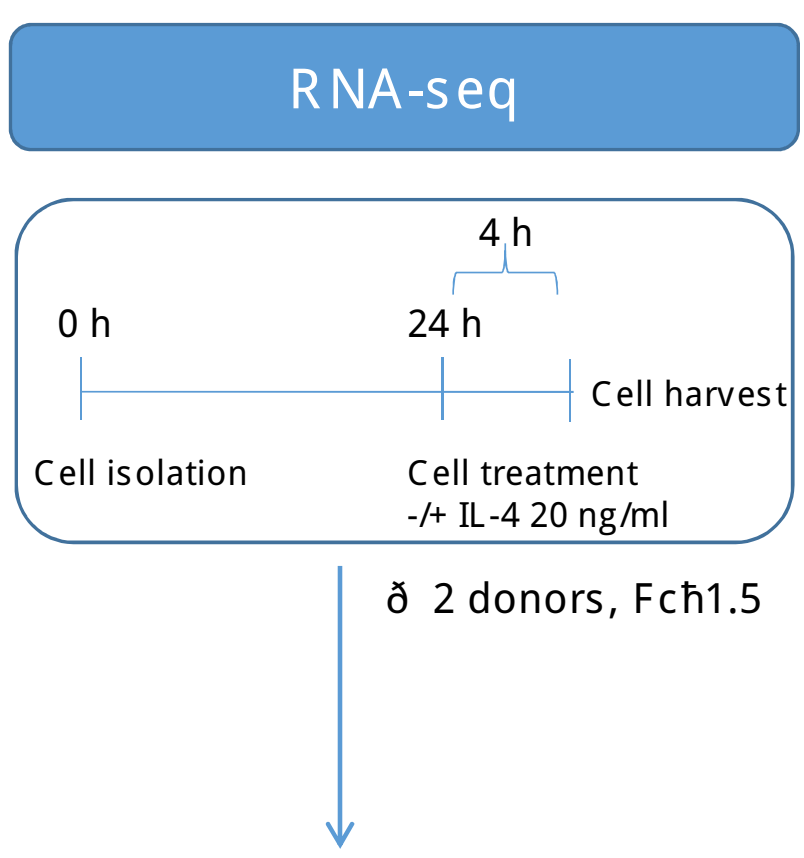
ðL-4-responsive macrophage
transcriptome 
A

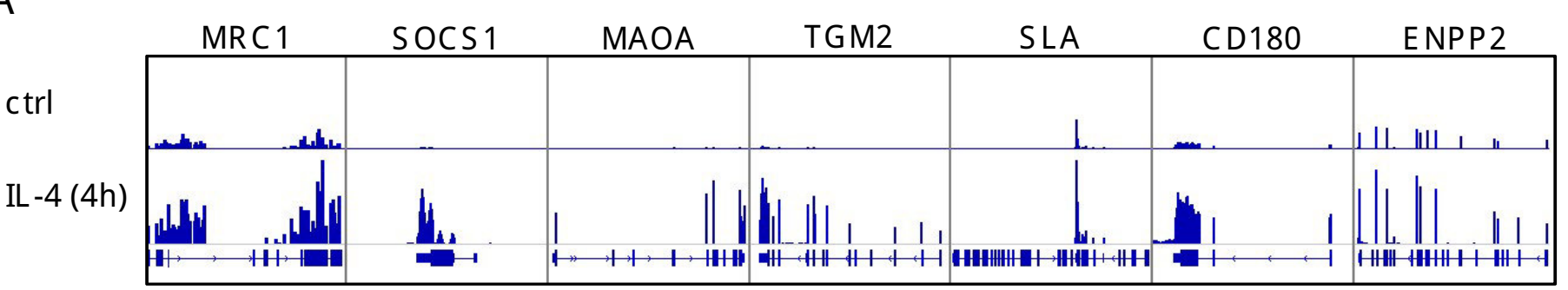

B

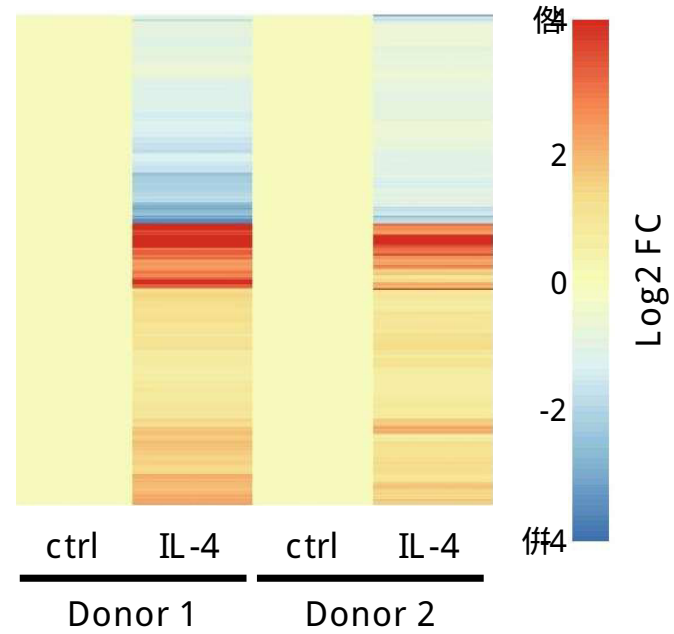

D

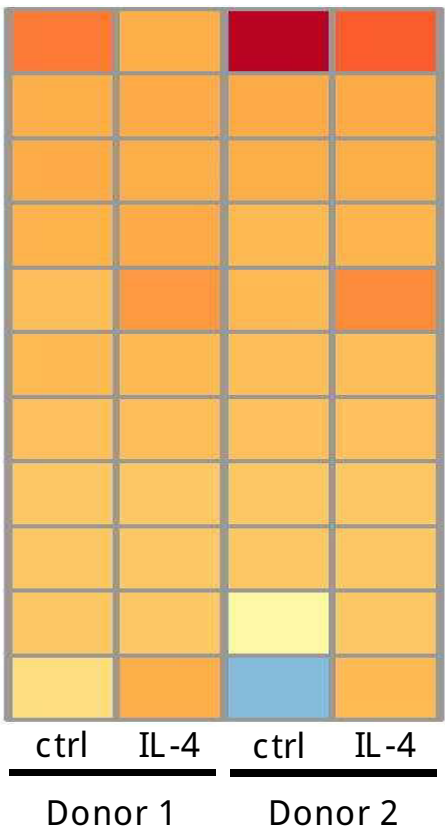

LXR

$\operatorname{LXR} \oint$

$\mathrm{RXR}$

PPAR 200

VDR

RXR $\oint$

RAR 100 주준

THR

PPAR 50

RAR $\therefore$

PPAR $\therefore$

C

\begin{tabular}{l}
1 \\
$\sim$ \\
0 \\
0 \\
\hline
\end{tabular}

Biological process Gene No. p-value FDR

$\begin{array}{cccc}\begin{array}{c}\text { inflammatory response } \\ \text { positive regulation of } \\ \text { inflammatory response } \\ \text { immune response }\end{array} & 56 & 1.27 \mathrm{E}-16 & 2.00 \mathrm{E}-13 \\ \begin{array}{c}\text { cellular response to } \\ \text { interferon-gamma } \\ \text { chemotaxis }\end{array} & 16 & 6.12 \mathrm{E}-11 & 1.11 \mathrm{E}-07 \\ \begin{array}{c}\text { chemokine-mediated } \\ \text { signaling pathway }\end{array} & 16 & 3.41 \mathrm{E}-09 & 6.20 \mathrm{E}-06 \\ \begin{array}{c}\text { response to } \\ \text { lipopolysaccharide } \\ \text { signal transduction }\end{array} & 24 & 1.33 \mathrm{E}-07 & 2.41 \mathrm{E}-04 \\ \text { monocyte chemotaxis } & 12 & 9.89 \mathrm{E}-07 & 4.03 \mathrm{E}-04 \\ \begin{array}{c}\text { lymphocyte chemotaxis } \\ \text { lym }-06\end{array} & 10 & 1.01 \mathrm{E}-06 & 1.83 \mathrm{E}-03\end{array}$


A

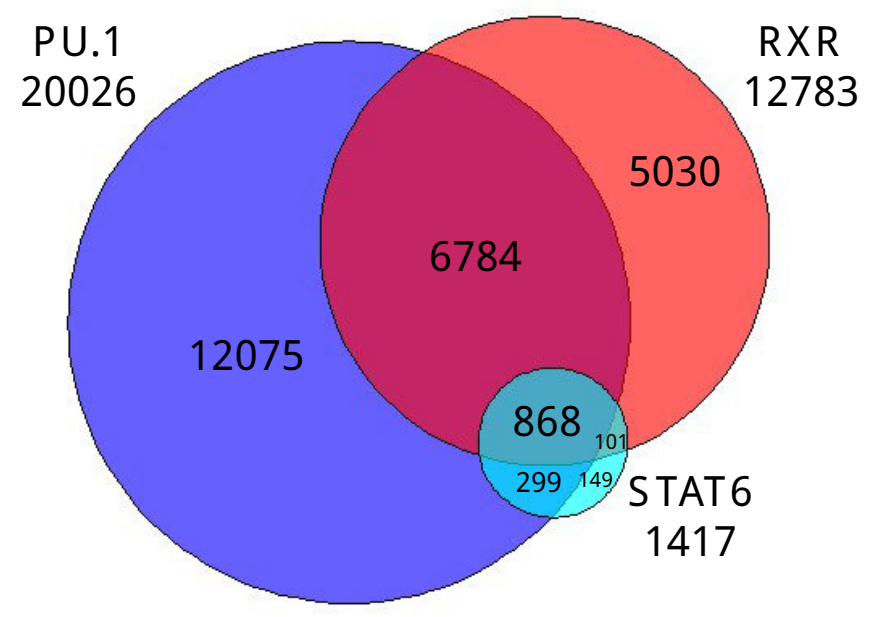

\begin{tabular}{|l|c|l|l|}
\hline Peaks (DiffBind) & PU.1 & RXR & STAT6 \\
\hline Consensus & 26531 & 14125 & 1573 \\
\hline Active (H3K27ac) & 20046 & 12799 & 1417 \\
\hline
\end{tabular}

C

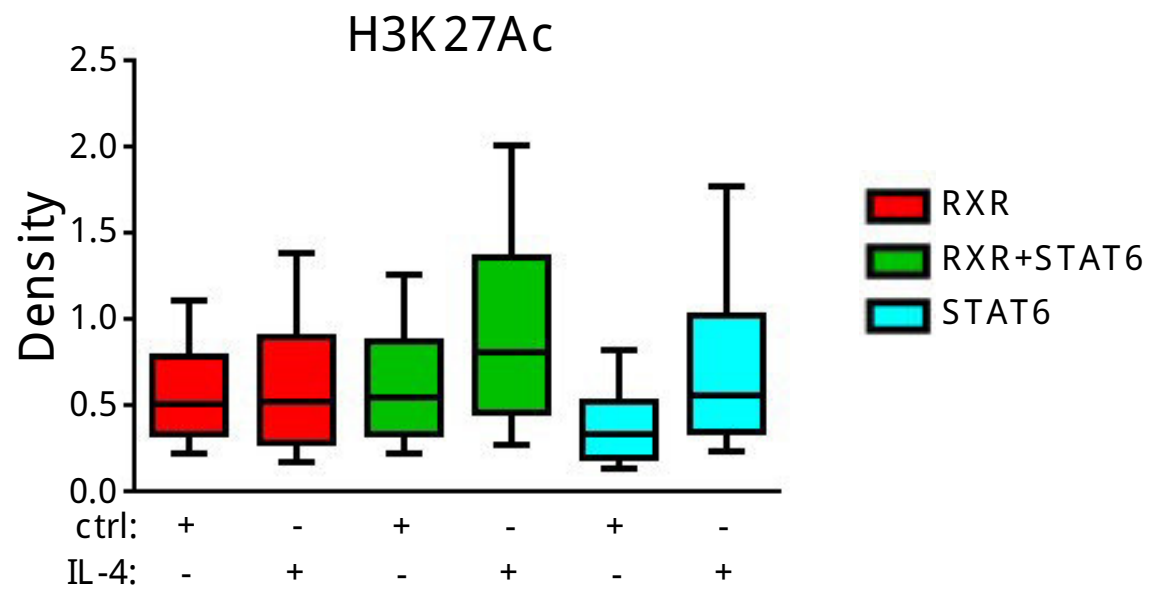

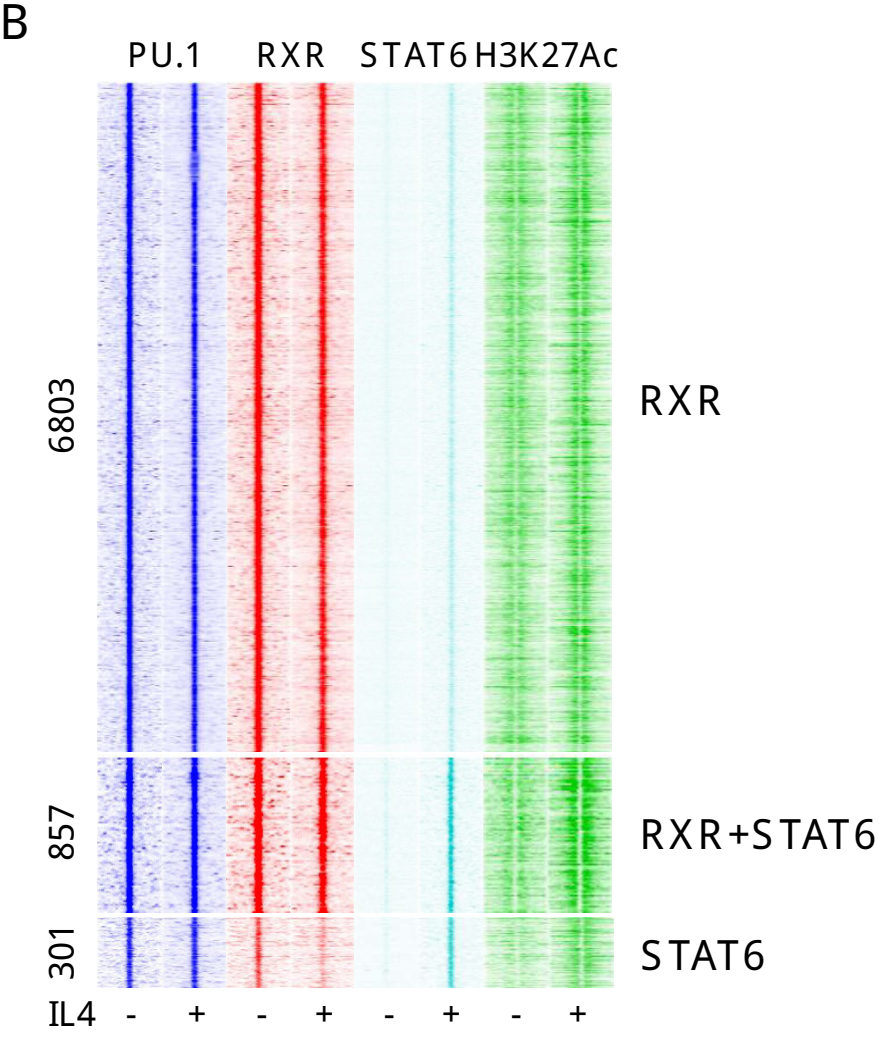


A
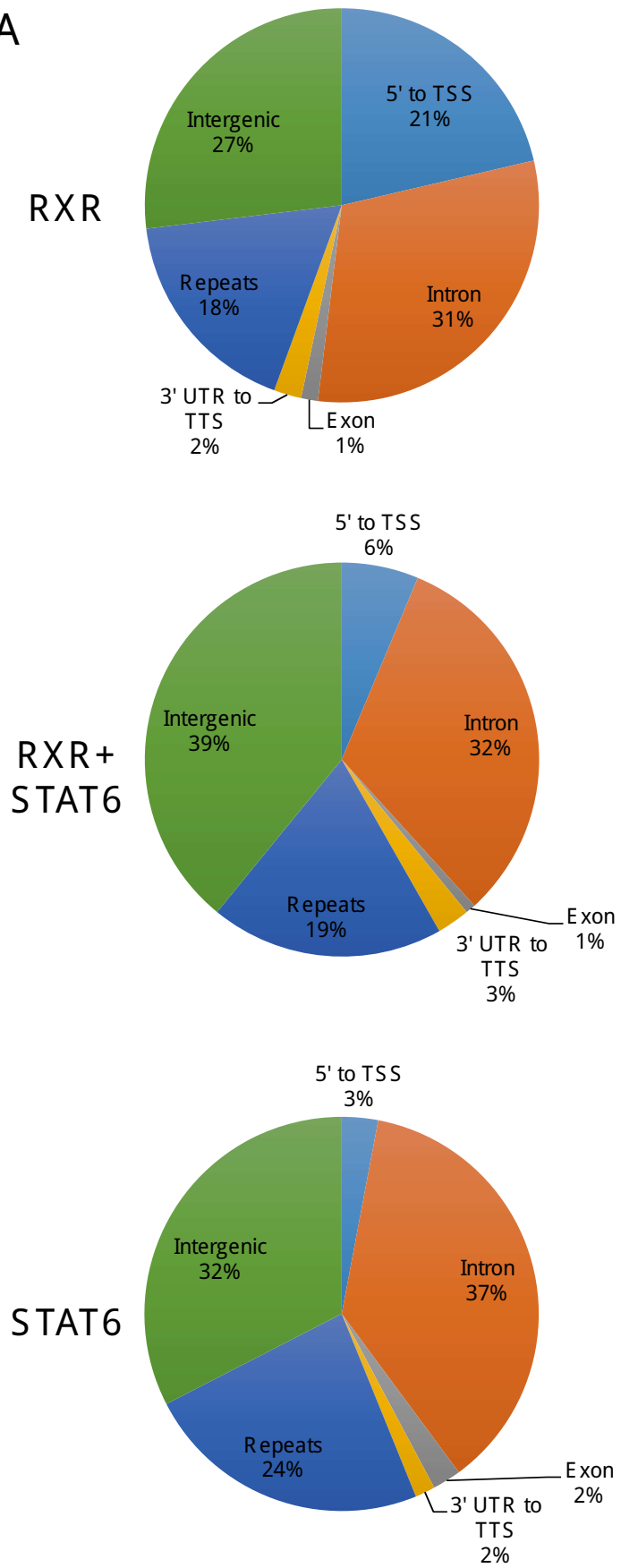

B

\begin{tabular}{|c|c|c|c|c|}
\hline \multicolumn{5}{|c|}{$\begin{array}{l}\text { PU.1 + RXR } \\
(883 / 1000)\end{array}$} \\
\hline & p-value & Target (\%) & $\mathrm{Bg}(\%)$ & Motif \\
\hline$\therefore=G G A A s x$ & $1 \mathrm{E}-278$ & 63.19 & 12.12 & PU.1 \\
\hline$T_{x} x_{x} \mathrm{CAA}$ & $1 \mathrm{E}-75$ & 16.53 & 2.31 & C/EBP \\
\hline$G G T T A C T=E G G T C A=$ & $1 \mathrm{E}-71$ & 2.94 & 0 & DR4 \\
\hline$T_{\subseteq A_{\varepsilon}} T C A$ & $1 \mathrm{E}-62$ & 20.84 & 4.78 & AP-1 \\
\hline$=\pi G A G_{C} T C A$ & $1 \mathrm{E}-41$ & 27.75 & 11.09 & NR half \\
\hline \multicolumn{5}{|c|}{$\begin{array}{c}\text { PU.1 + RXR + STAT 6 } \\
(703 / 857)\end{array}$} \\
\hline & p-value & Target (\%) & $\mathrm{Bg}(\%)$ & Motif \\
\hline$\ldots G G A A_{-T}$ & $1 \mathrm{E}-247$ & 52.54 & 6.21 & PU.1 \\
\hline $\mathrm{TT}_{\mathrm{a}}^{\mathrm{a} A}$ & $1 E-124$ & 36.76 & 6.31 & C/EBP \\
\hline TTc $=\mathrm{GAA}$ & $1 \mathrm{E}-92$ & 36.48 & 8.63 & STAT6 \\
\hline$=$ TGAeTCA & $1 E-38$ & 20.99 & 6.2 & AP-1 \\
\hline$G G G_{A \cap T T T} T C_{c}$ & $1 E-26$ & 21.27 & 8.28 & REL \\
\hline$\Rightarrow A A C C A C$ & $1 \mathrm{E}-22$ & 10.99 & 2.86 & RUNX \\
\hline \multicolumn{5}{|c|}{$\begin{array}{l}\text { PU.1 + STAT } 6 \\
(233 / 301)\end{array}$} \\
\hline & p-value & Target (\%) & $\mathrm{Bg}(\%)$ & Motif \\
\hline$G G A A_{\cong} T$ & $1 \mathrm{E}-80$ & 61.8 & 10.25 & PU.1 \\
\hline$-T=C_{z}=G A A_{-}$ & $1 \mathrm{E}-57$ & 45.06 & 6.96 & STAT6 \\
\hline${ }_{\triangle} T_{\mathrm{sT}} \mathrm{G}_{s} \mathrm{AA} I$ & $1 \mathrm{E}-22$ & 28.76 & 7.12 & $C / E B P$ \\
\hline a TGA ICA $=$ & $1 \mathrm{E}-18$ & 16.74 & 2.72 & $A P-1$ \\
\hline
\end{tabular}


A

IL -4 responsive genes-associated

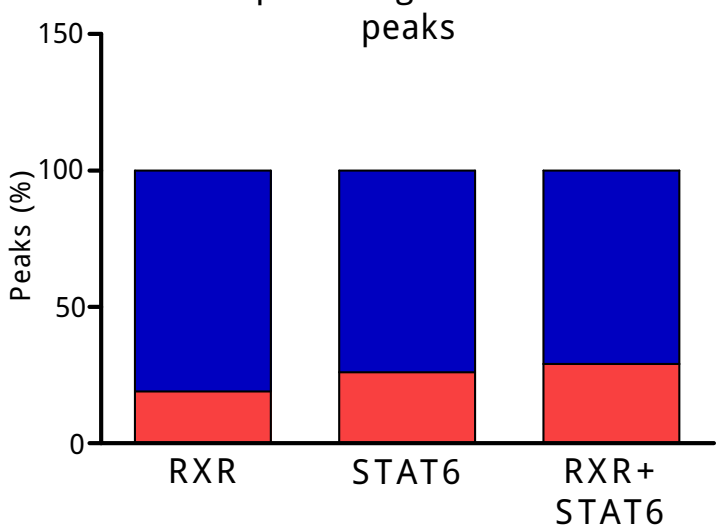

B

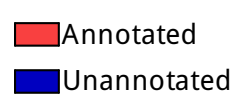

Peak distribution relative to TSS

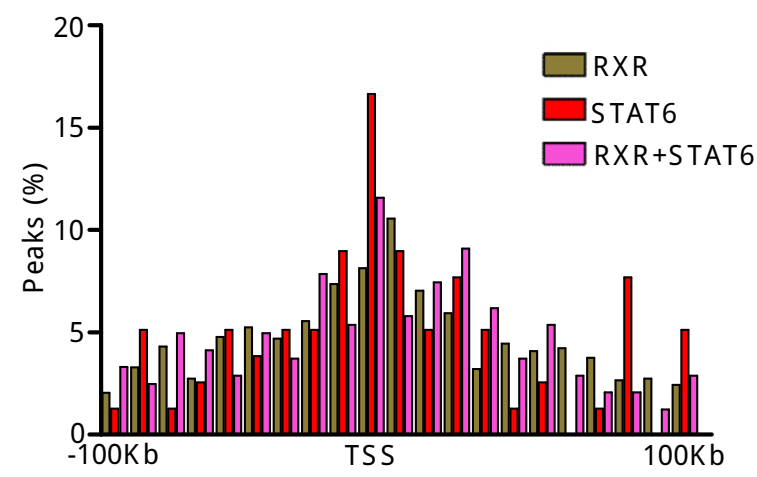

C

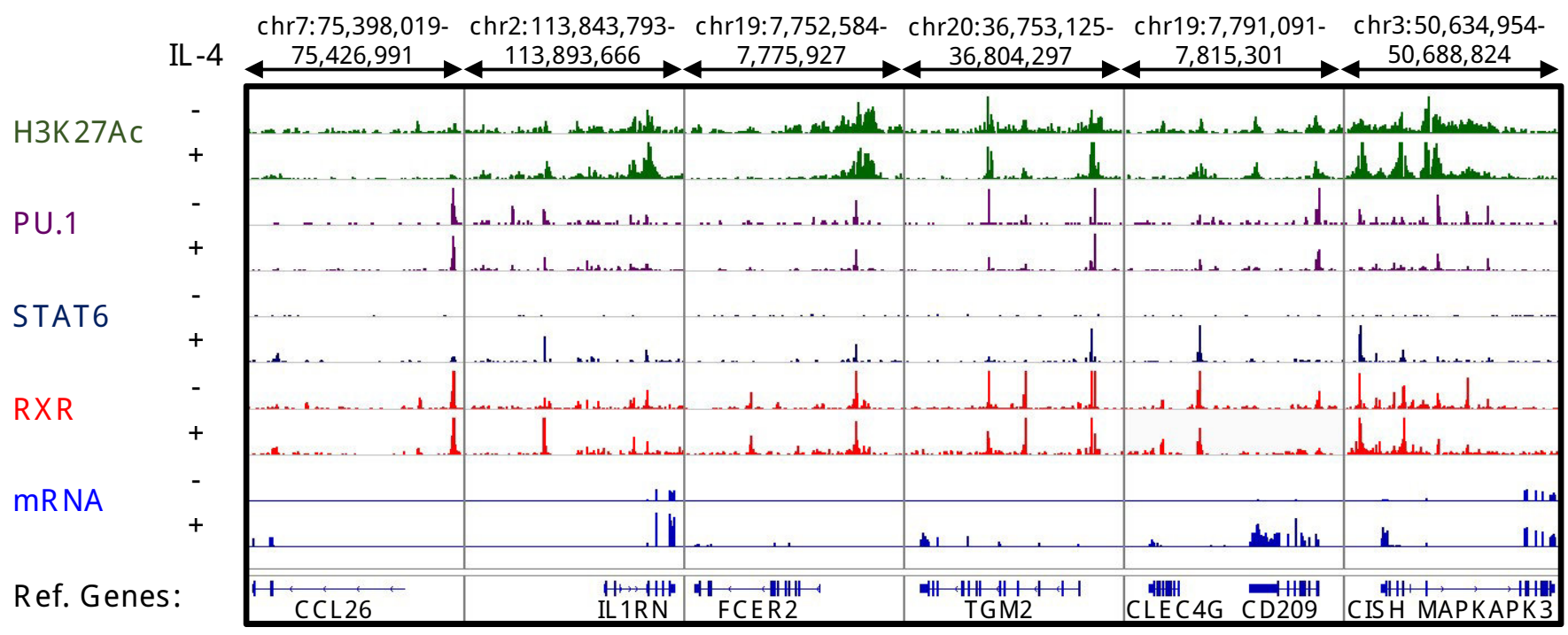


A

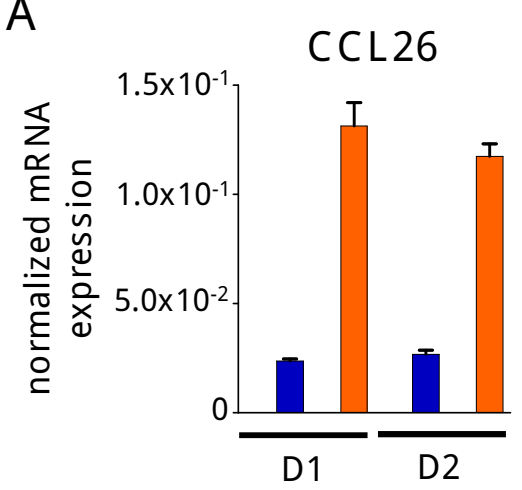

IL 1 R N

FCER2 TGM2
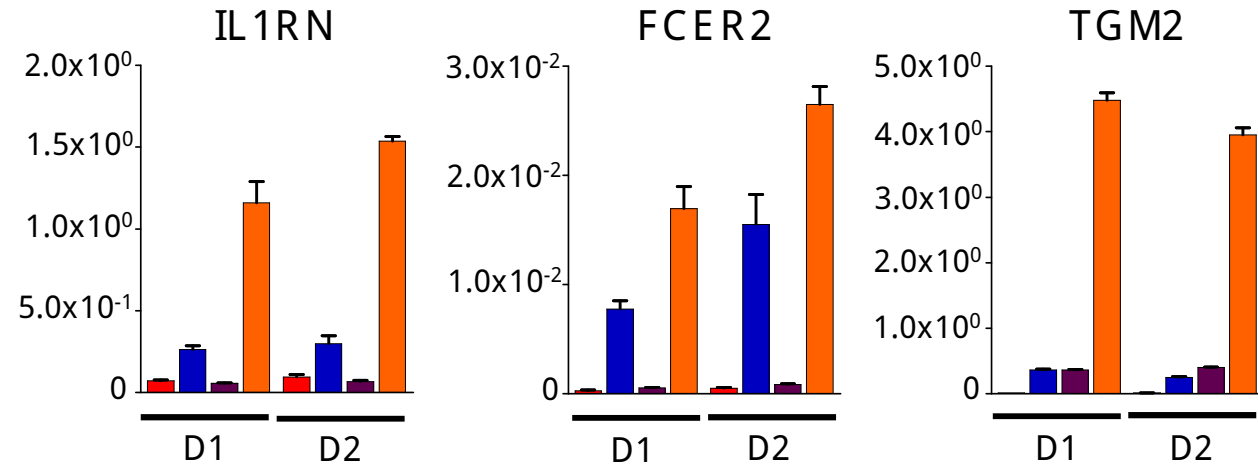

CLEC4G

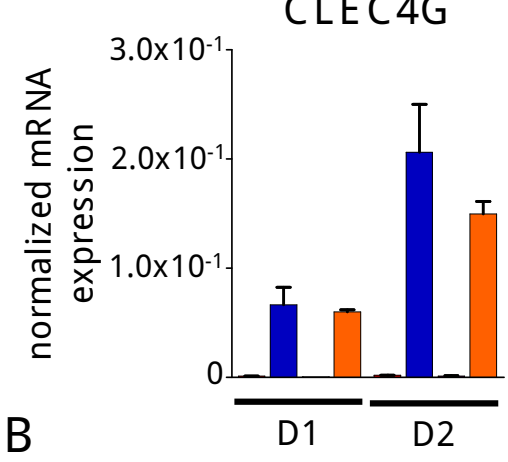

CD209
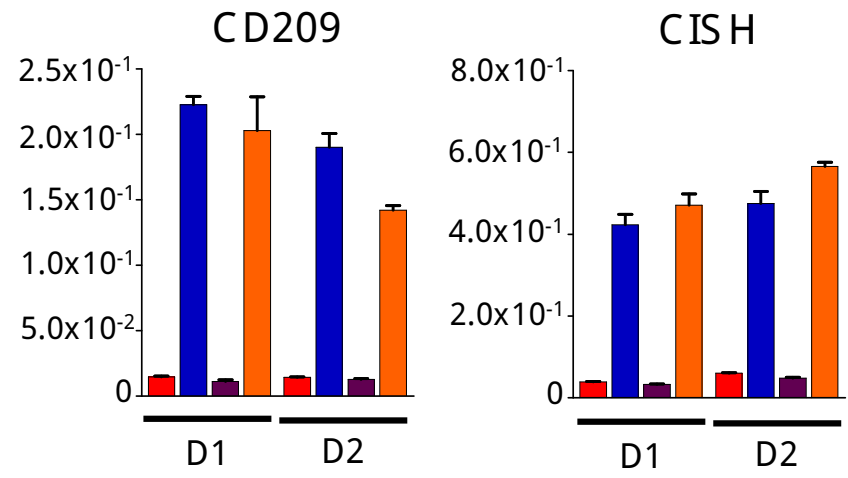

$\square$ veh

CCL26_-6,5Kb

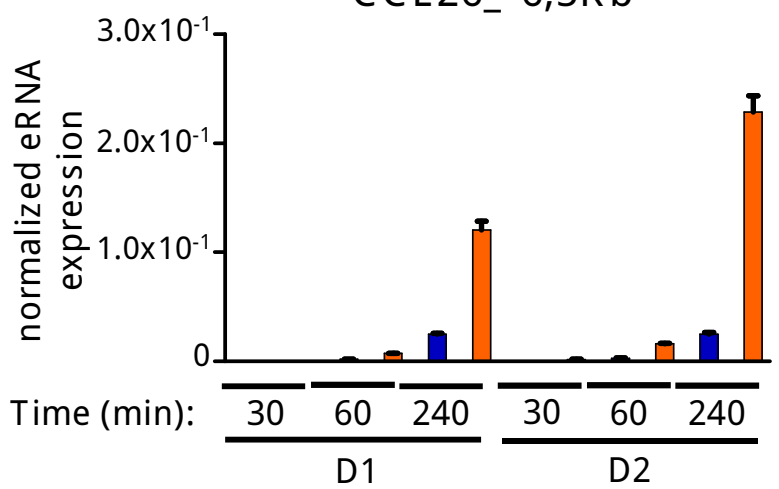

IL $1 R N_{-}-13 K b$

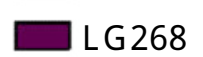

IL-4/L G 268

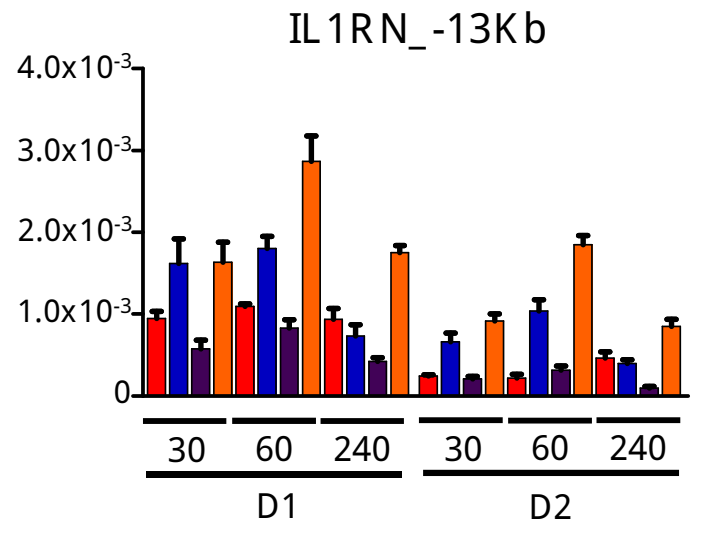

$\square$ veh

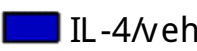

$\square$ L G 268

$\square$ IL-4/L G 268

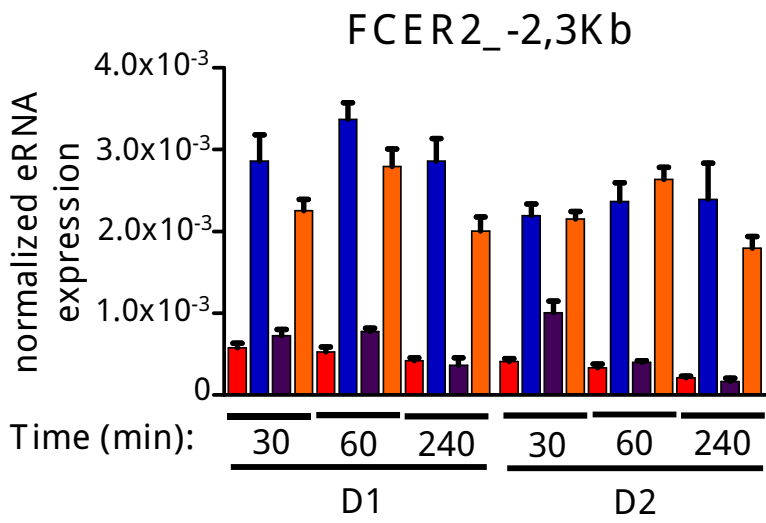

TGM2_-3,3Kb
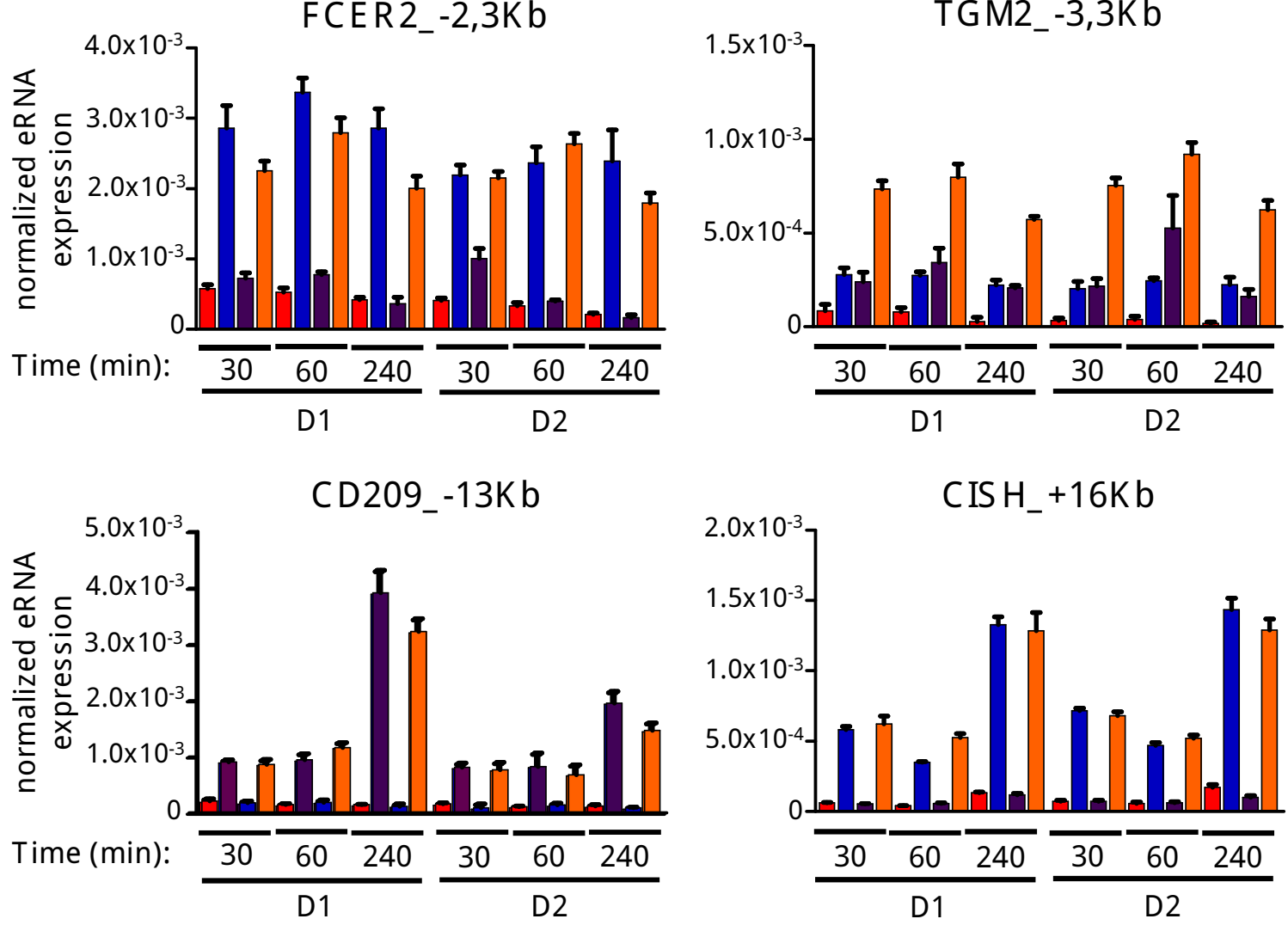
synergistic

potentiating

liganded RXR independent

veh
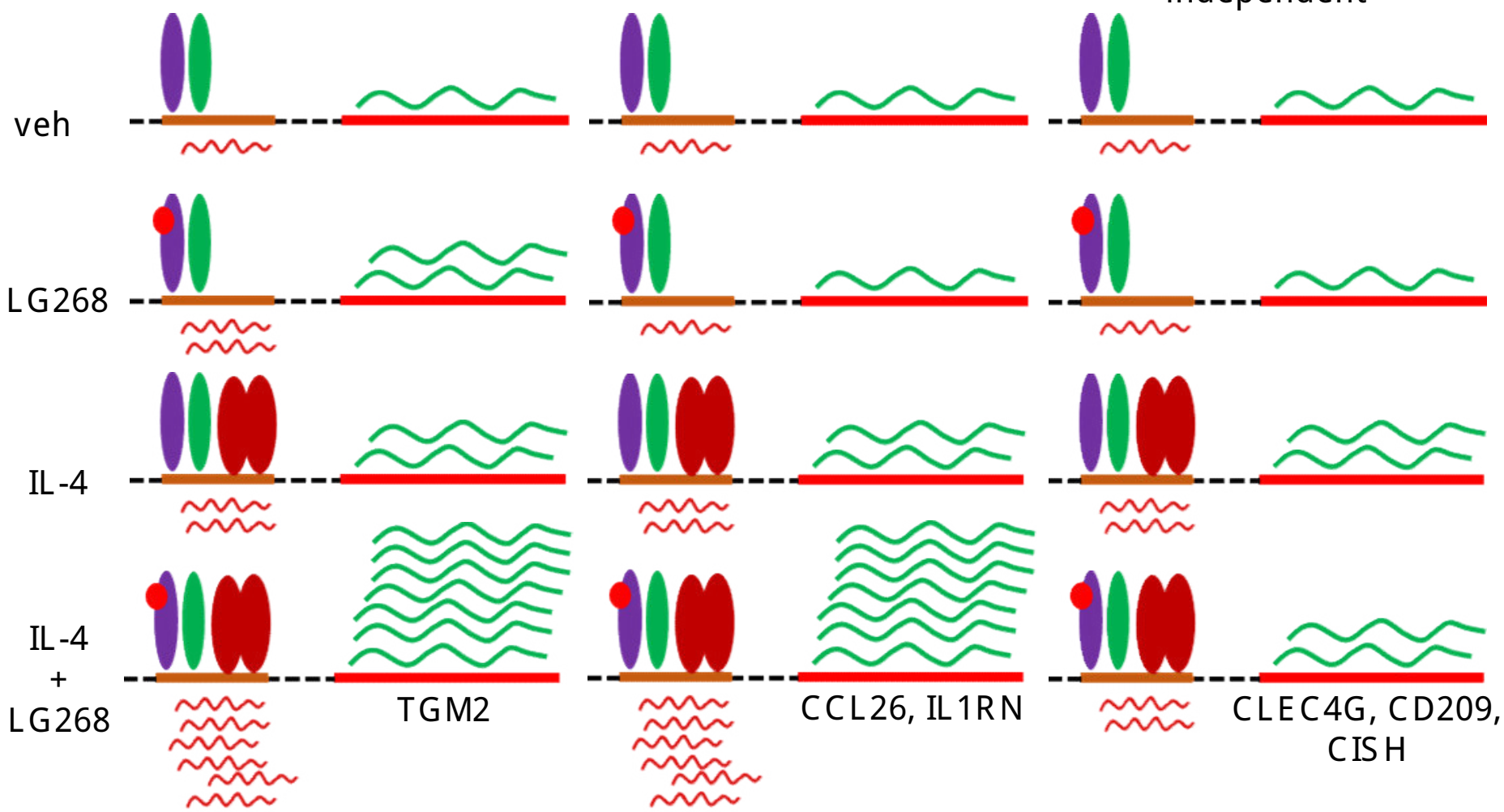

$\sim$ mRNA $\sim$ eRNA RXR NRR STAT6 LG268 
A

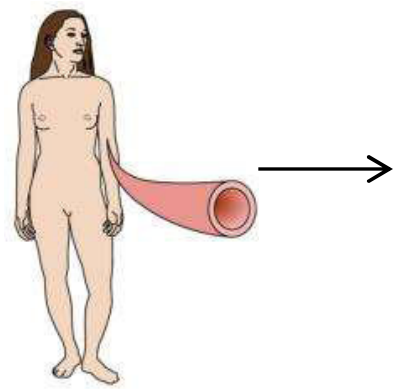

Buffy coat
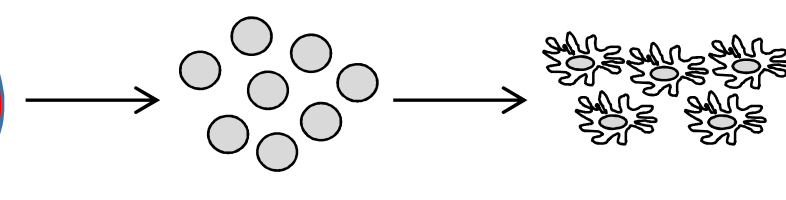

\section{象}

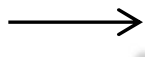

Veh

Magnetic separation of CD14+ monocytes

B
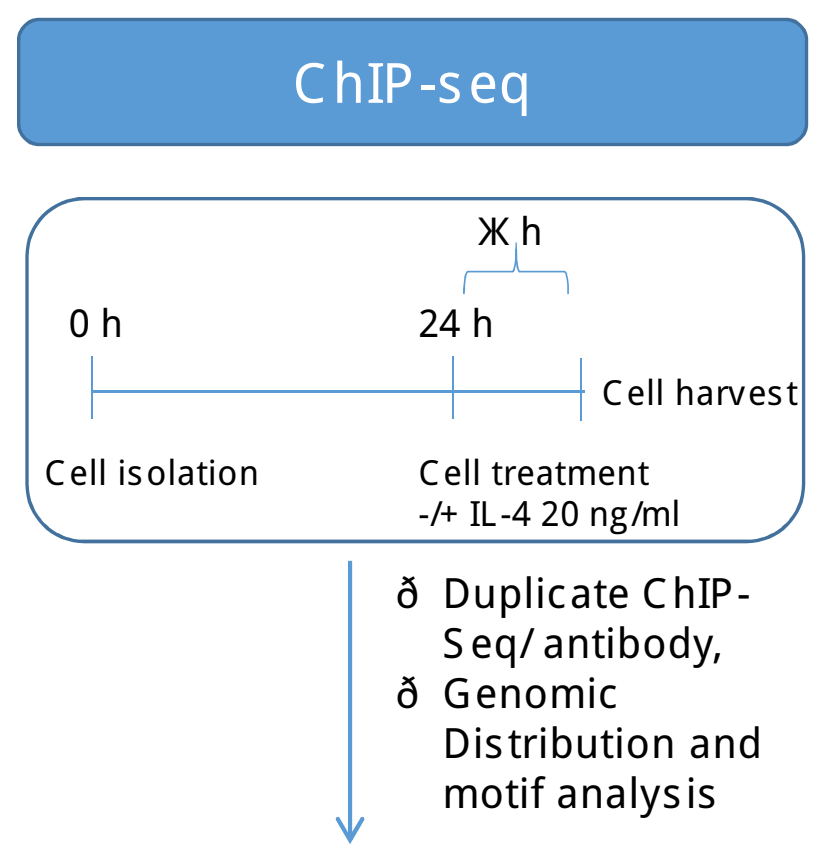

ð human macrophge-specific $\left(\mathrm{H} 3 \mathrm{~K} 27 \mathrm{Ac}+/ \mathrm{PU} .1^{+}\right)$enhancer set

ð STAT6 cistrome

ð RXR cistrome

ð RXR/STAT6 co-peaks
$24 \mathrm{~h}$ attachment on culture dish
Cell treatments

IL -4
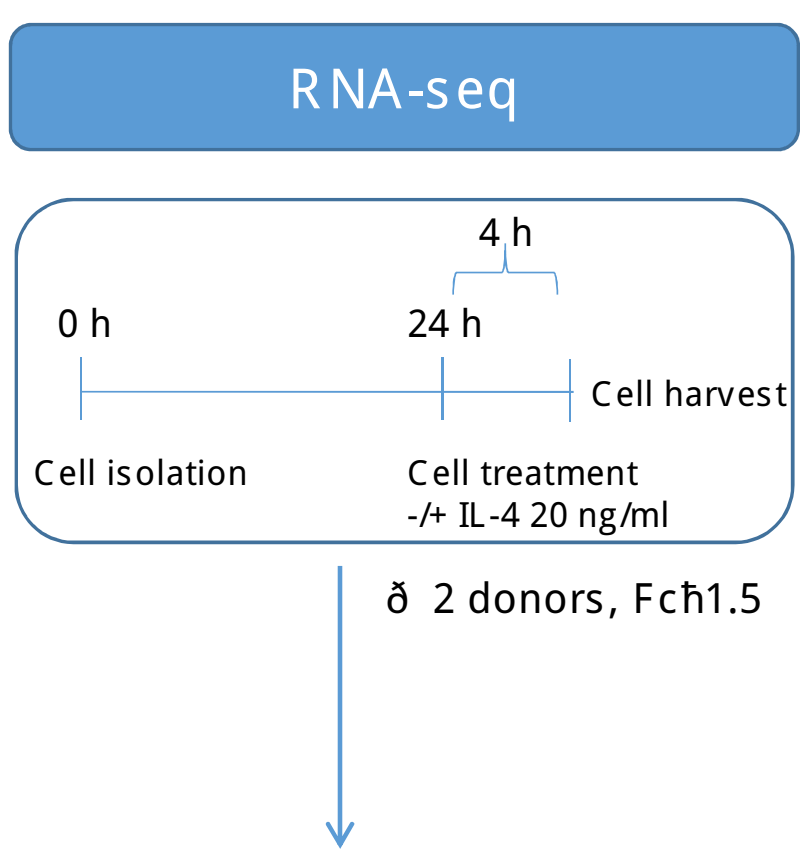
ðL-4-responsive macrophage
transcriptome 
A

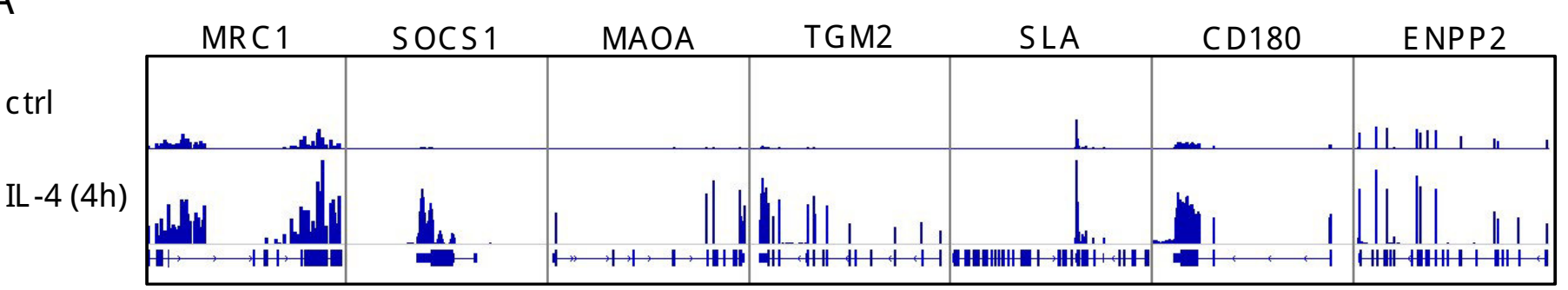

B

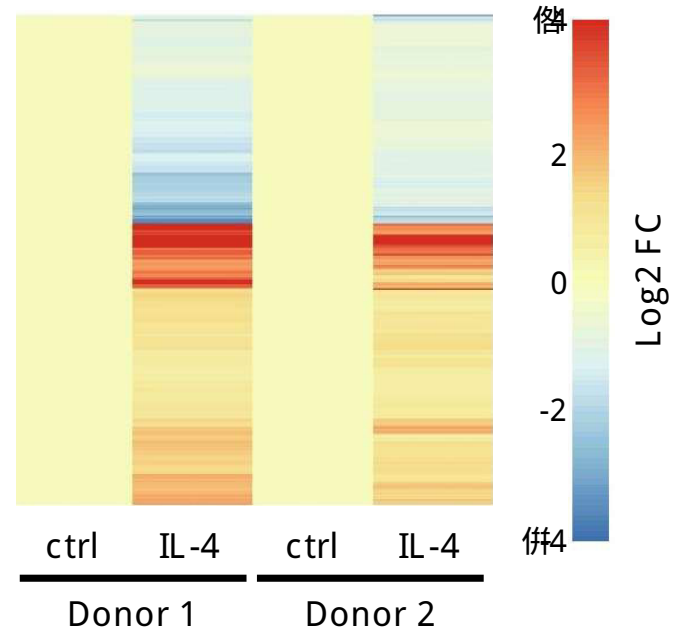

D

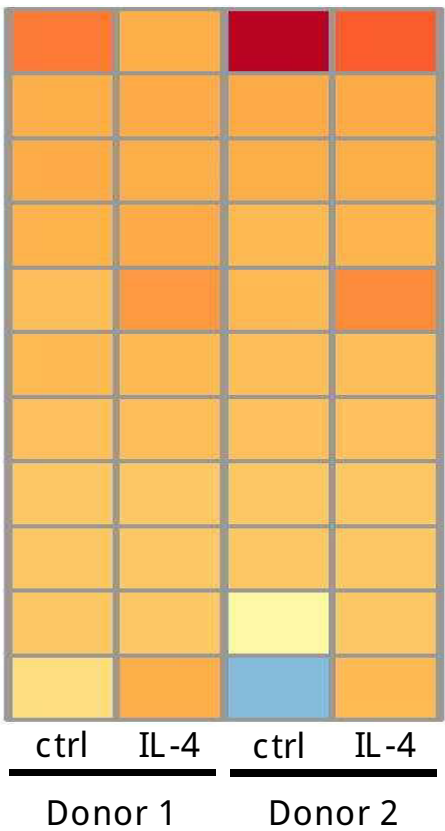

LXR

$\operatorname{LXR} \oint$

$\mathrm{RXR}$

PPAR 200

VDR

RXR $\oint$

RAR 100 주준

THR

PPAR 50

RAR $\therefore$

PPAR $\therefore$

C

\begin{tabular}{l}
1 \\
$\sim$ \\
0 \\
0 \\
\hline
\end{tabular}

Biological process Gene No. p-value FDR

$\begin{array}{cccc}\begin{array}{c}\text { inflammatory response } \\ \text { positive regulation of } \\ \text { inflammatory response } \\ \text { immune response }\end{array} & 56 & 1.27 \mathrm{E}-16 & 2.00 \mathrm{E}-13 \\ \begin{array}{c}\text { cellular response to } \\ \text { interferon-gamma } \\ \text { chemotaxis }\end{array} & 16 & 6.12 \mathrm{E}-11 & 1.11 \mathrm{E}-07 \\ \begin{array}{c}\text { chemokine-mediated } \\ \text { signaling pathway }\end{array} & 16 & 3.41 \mathrm{E}-09 & 6.20 \mathrm{E}-06 \\ \begin{array}{c}\text { response to } \\ \text { lipopolysaccharide } \\ \text { signal transduction }\end{array} & 24 & 1.33 \mathrm{E}-07 & 2.41 \mathrm{E}-04 \\ \text { monocyte chemotaxis } & 12 & 9.89 \mathrm{E}-07 & 4.03 \mathrm{E}-04 \\ \begin{array}{c}\text { lymphocyte chemotaxis } \\ \text { lym }-06\end{array} & 10 & 1.01 \mathrm{E}-06 & 1.83 \mathrm{E}-03\end{array}$


A

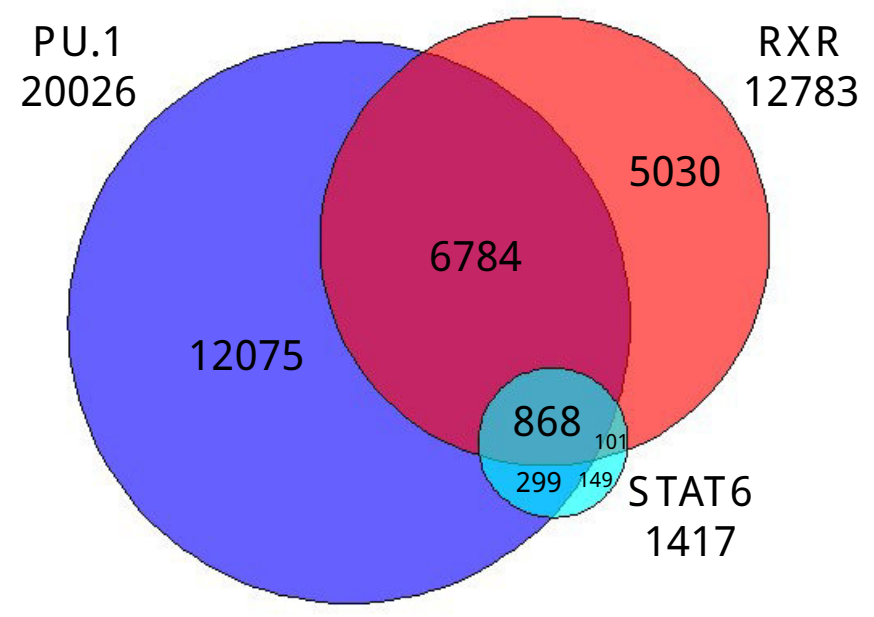

\begin{tabular}{|l|c|l|l|}
\hline Peaks (DiffBind) & PU.1 & RXR & STAT6 \\
\hline Consensus & 26531 & 14125 & 1573 \\
\hline Active (H3K27ac) & 20046 & 12799 & 1417 \\
\hline
\end{tabular}

C

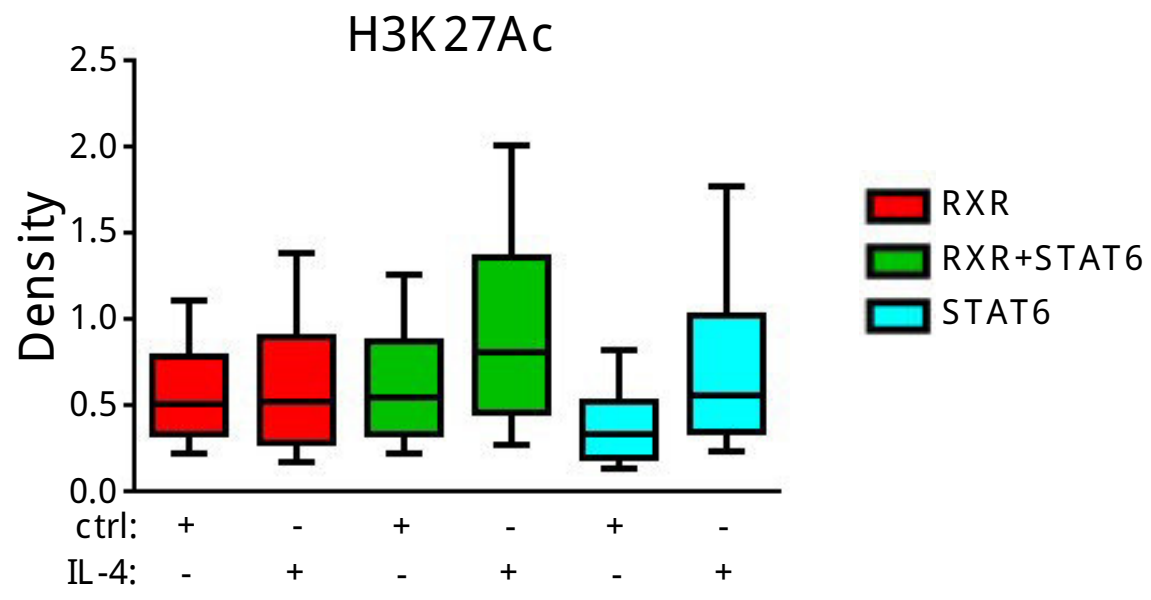

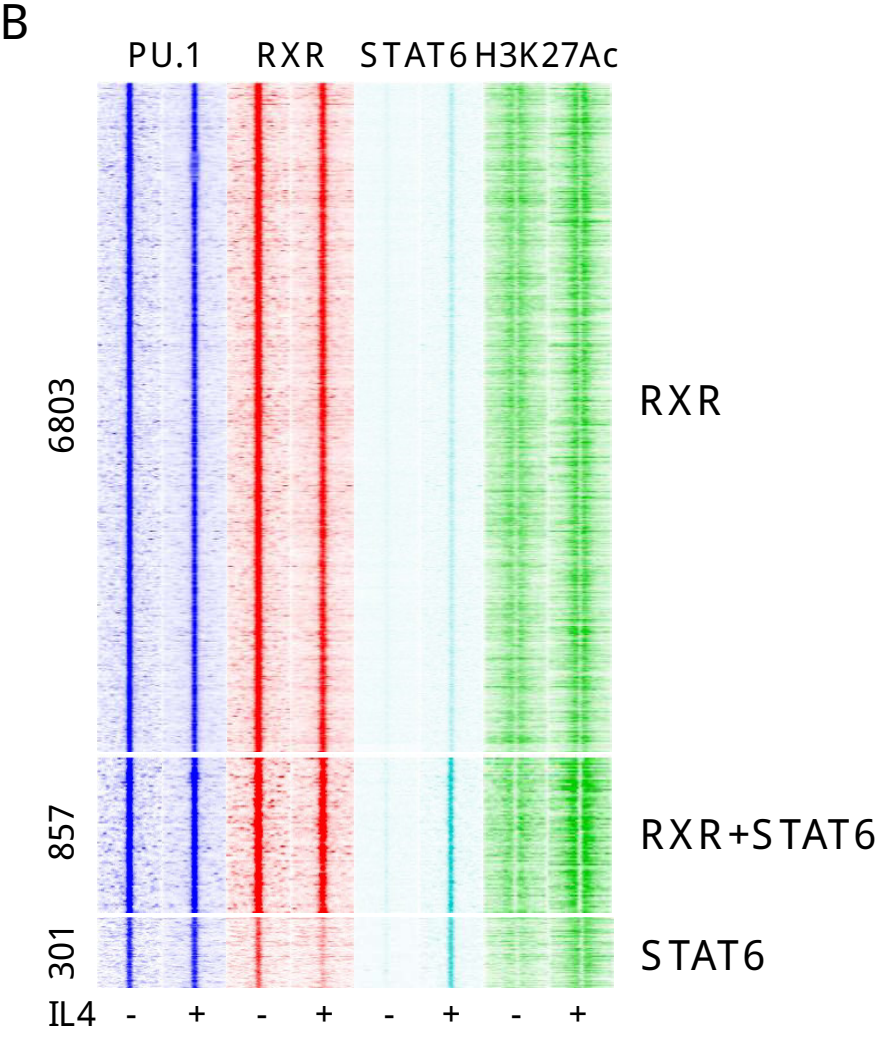


A
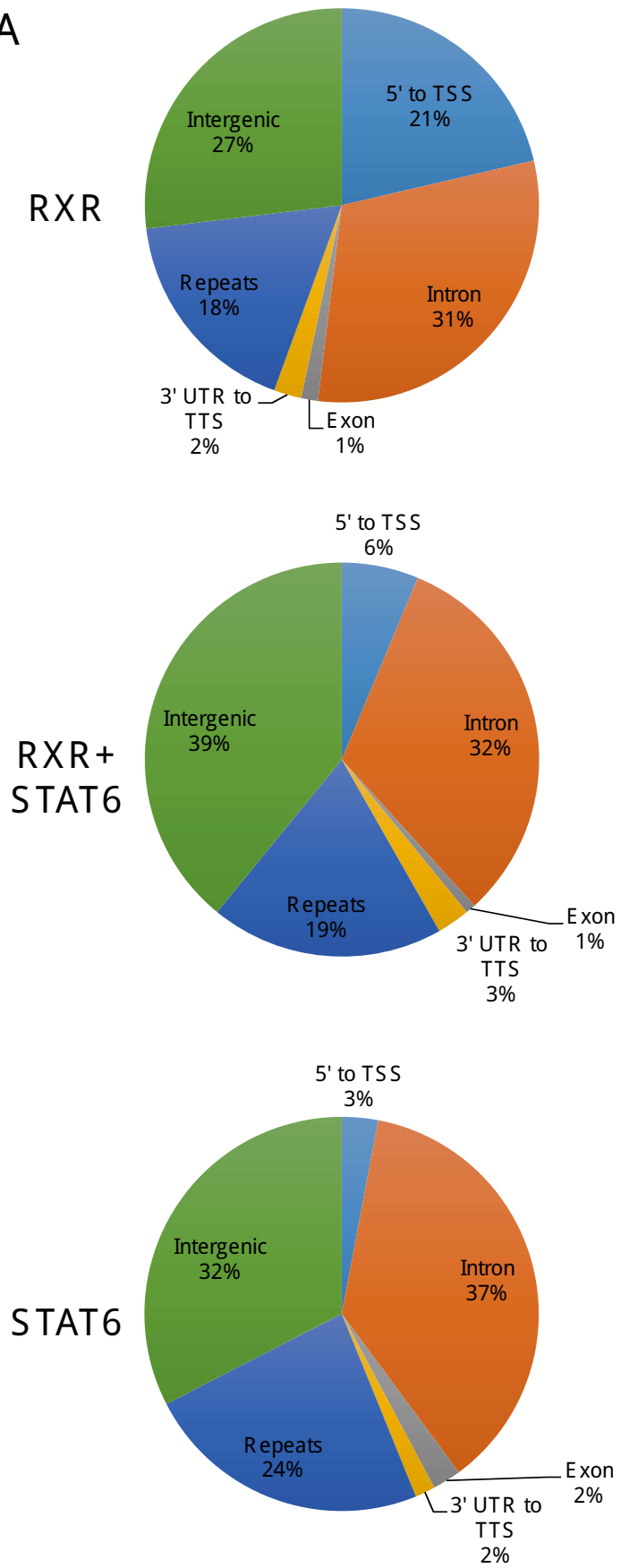

B

\begin{tabular}{|c|c|c|c|c|}
\hline \multicolumn{5}{|c|}{$\begin{array}{l}\text { PU.1 + RXR } \\
(883 / 1000)\end{array}$} \\
\hline & p-value & Target (\%) & $\mathrm{Bg}(\%)$ & Motif \\
\hline$\therefore=G G A A s x$ & $1 \mathrm{E}-278$ & 63.19 & 12.12 & PU.1 \\
\hline$T_{x} x_{x} \mathrm{CAA}$ & $1 \mathrm{E}-75$ & 16.53 & 2.31 & C/EBP \\
\hline$G G T T A C T=E G G T C A=$ & $1 \mathrm{E}-71$ & 2.94 & 0 & DR4 \\
\hline$T_{\subseteq A_{\varepsilon}} T C A$ & $1 \mathrm{E}-62$ & 20.84 & 4.78 & AP-1 \\
\hline$=\pi G A G_{C} T C A$ & $1 \mathrm{E}-41$ & 27.75 & 11.09 & NR half \\
\hline \multicolumn{5}{|c|}{$\begin{array}{c}\text { PU.1 + RXR + STAT 6 } \\
(703 / 857)\end{array}$} \\
\hline & p-value & Target (\%) & $\mathrm{Bg}(\%)$ & Motif \\
\hline$\ldots G G A A_{-T}$ & $1 \mathrm{E}-247$ & 52.54 & 6.21 & PU.1 \\
\hline $\mathrm{TT}_{\mathrm{a}}^{\mathrm{a} A}$ & $1 E-124$ & 36.76 & 6.31 & C/EBP \\
\hline TTc $=\mathrm{GAA}$ & $1 \mathrm{E}-92$ & 36.48 & 8.63 & STAT6 \\
\hline$=$ TGAeTCA & $1 E-38$ & 20.99 & 6.2 & AP-1 \\
\hline$G G G_{A \cap T T T} T C_{c}$ & $1 E-26$ & 21.27 & 8.28 & REL \\
\hline$\Rightarrow A A C C A C$ & $1 \mathrm{E}-22$ & 10.99 & 2.86 & RUNX \\
\hline \multicolumn{5}{|c|}{$\begin{array}{l}\text { PU.1 + STAT } 6 \\
(233 / 301)\end{array}$} \\
\hline & p-value & Target (\%) & $\mathrm{Bg}(\%)$ & Motif \\
\hline$G G A A_{\cong} T$ & $1 \mathrm{E}-80$ & 61.8 & 10.25 & PU.1 \\
\hline$-T=C_{z}=G A A_{-}$ & $1 \mathrm{E}-57$ & 45.06 & 6.96 & STAT6 \\
\hline${ }_{\triangle} T_{\mathrm{sT}} \mathrm{G}_{s} \mathrm{AA} I$ & $1 \mathrm{E}-22$ & 28.76 & 7.12 & $C / E B P$ \\
\hline a TGA ICA $=$ & $1 \mathrm{E}-18$ & 16.74 & 2.72 & $A P-1$ \\
\hline
\end{tabular}


A

IL -4 responsive genes-associated

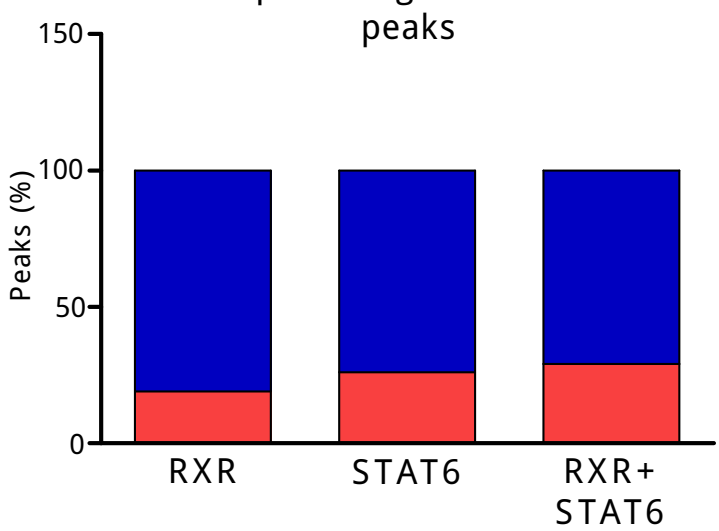

B

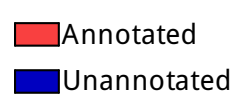

Peak distribution relative to TSS

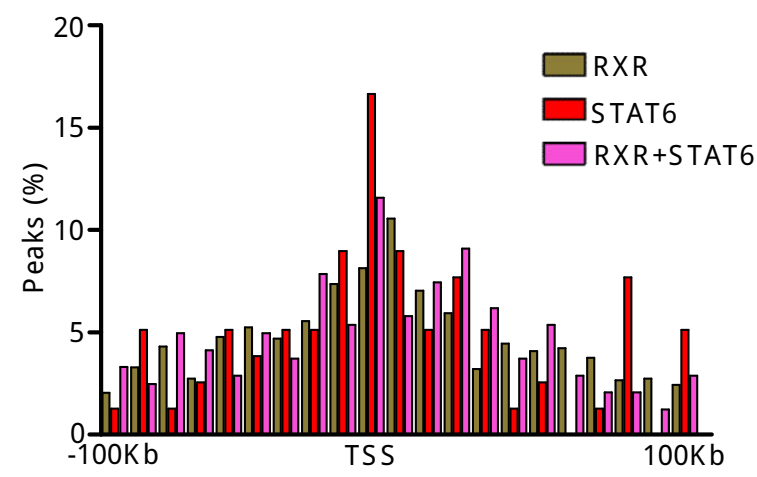

C

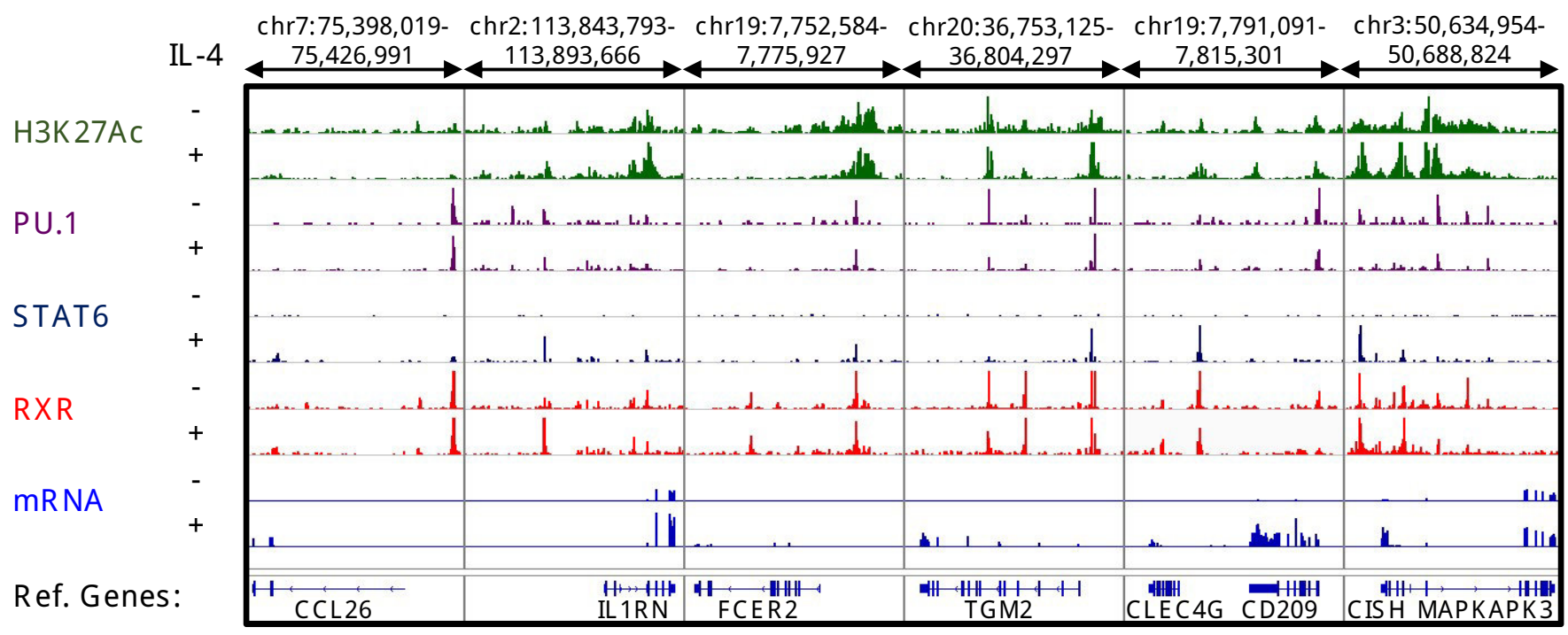


A

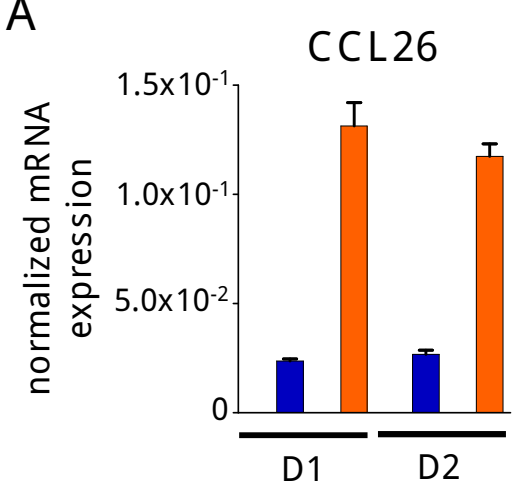

IL 1 R N

FCER2 TGM2
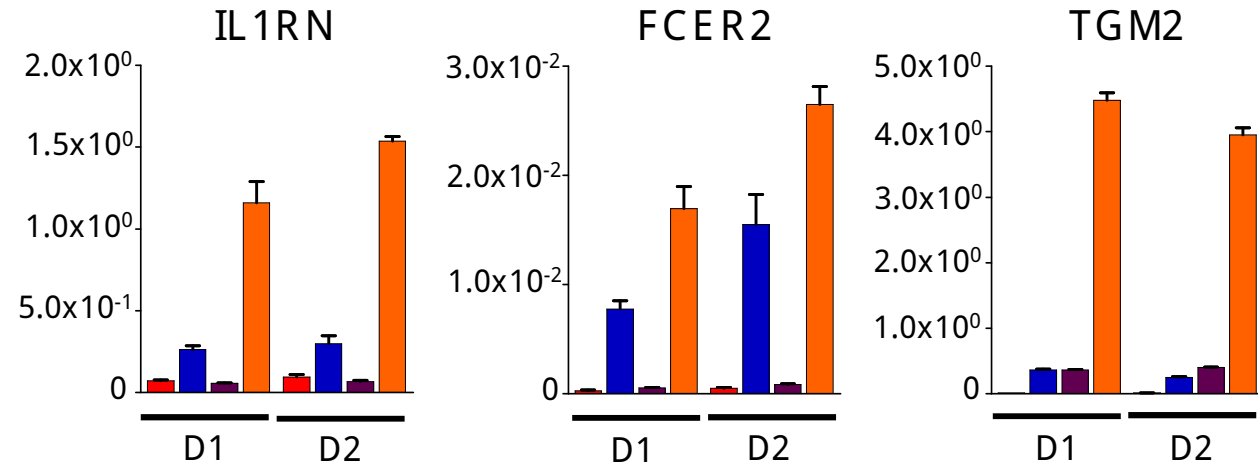

CLEC4G

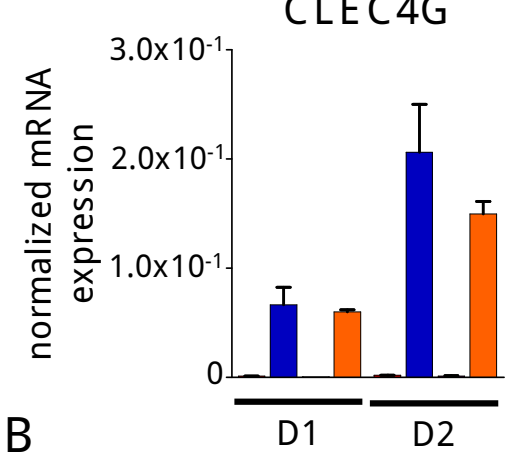

CD209
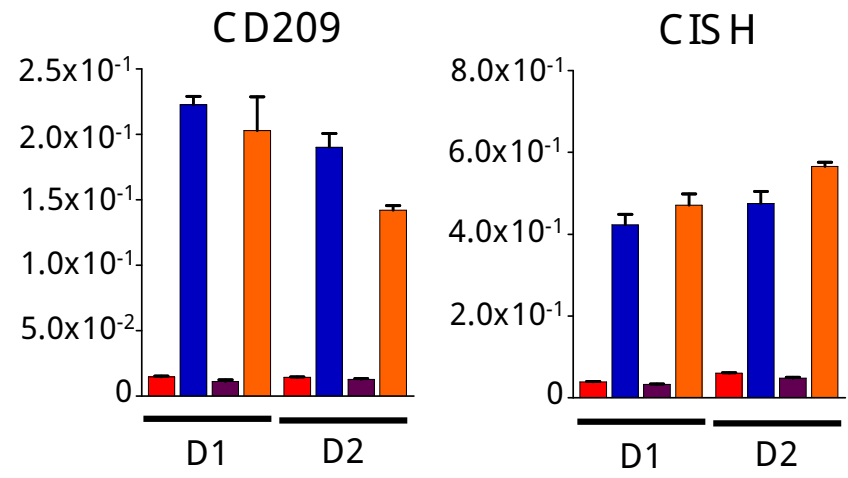

$\square$ veh

CCL26_-6,5Kb

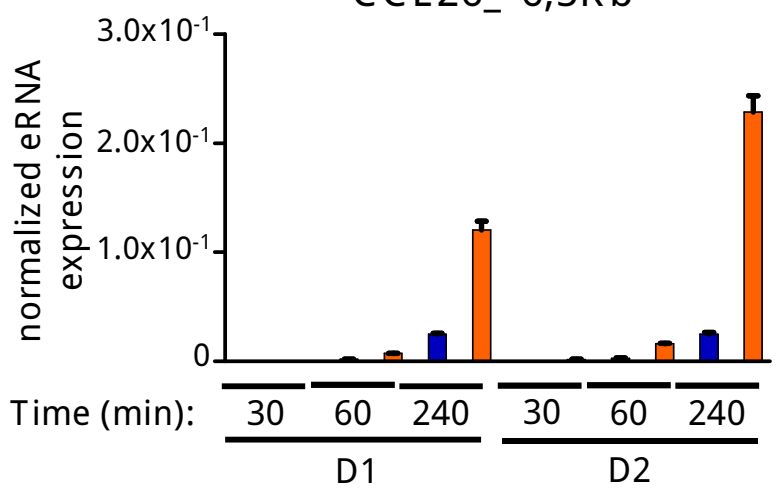

IL $1 R N_{-}-13 K b$

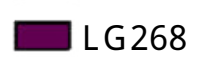

IL -4/L G 268

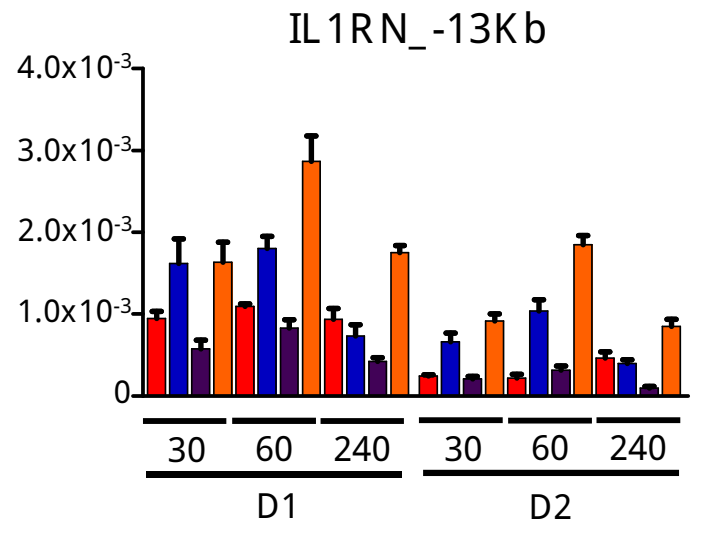

$\square$ veh

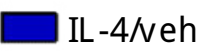

$\square$ L G 268

$\square$ IL-4/L G 268

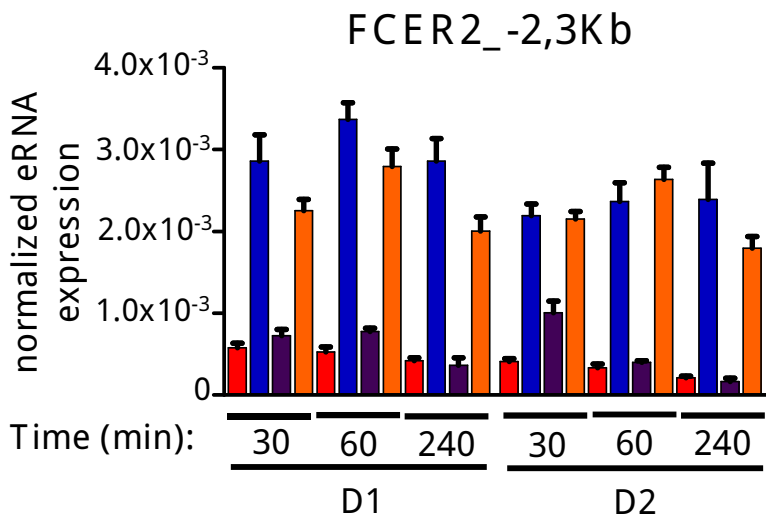

TGM2_-3,3Kb
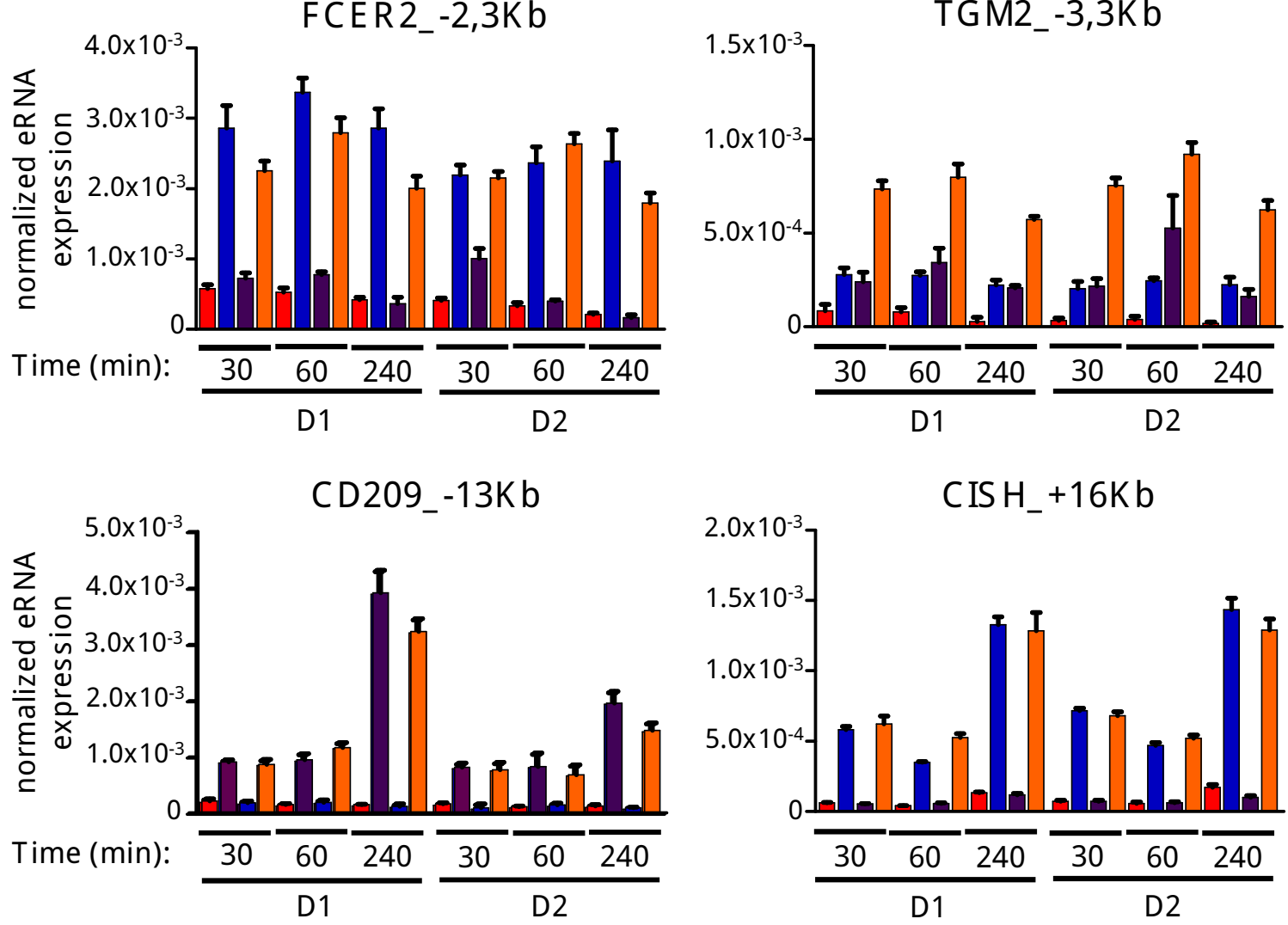
synergistic

potentiating

liganded RXR independent

veh
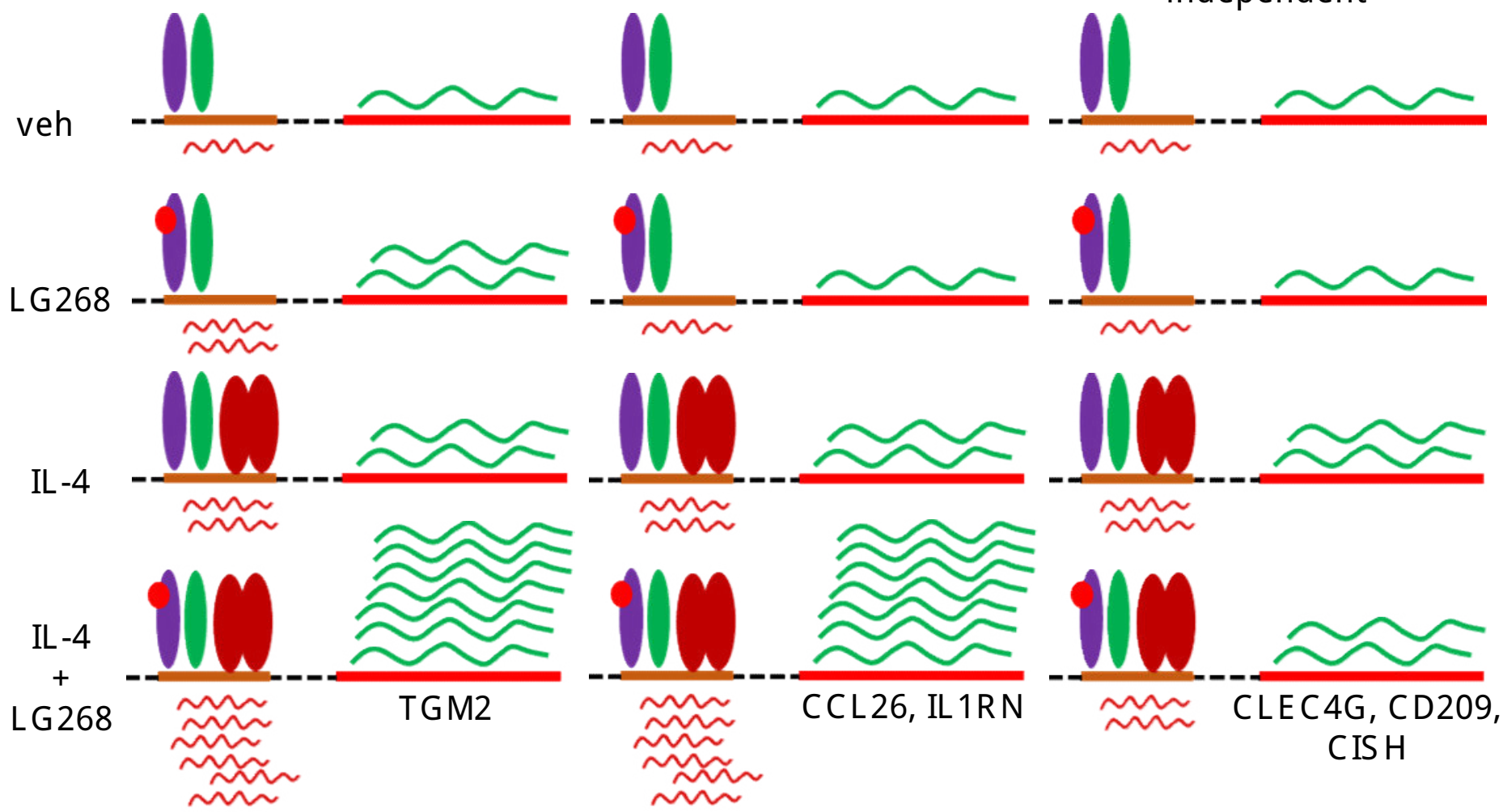

$\sim$ mRNA $\sim$ eRNA RXR NRR STAT6 LG268 
A

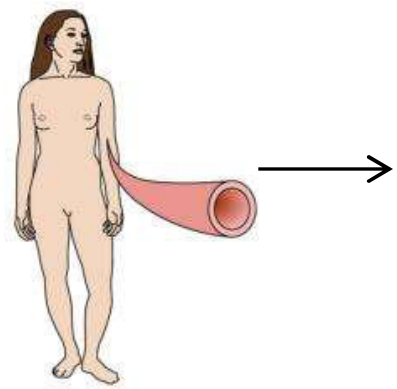

Buffy coat
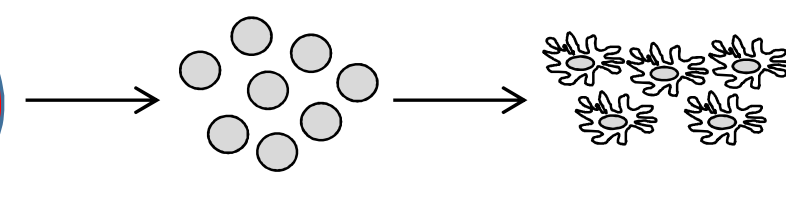

\section{象}

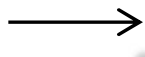

Veh

Magnetic separation of CD14+ monocytes

B
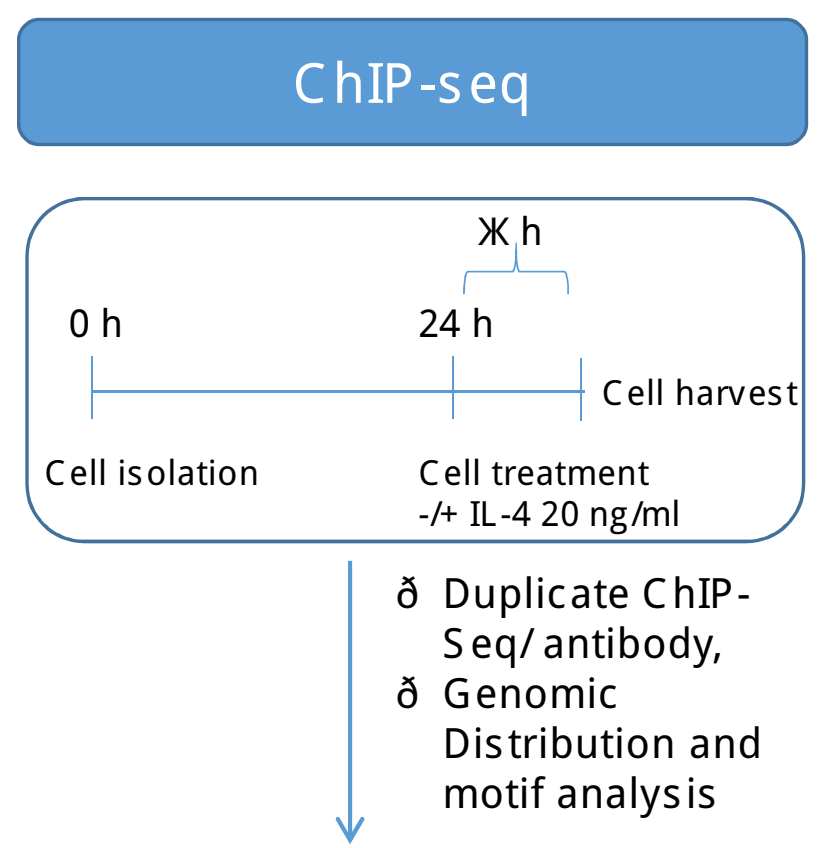

ð human macrophge-specific $\left(\mathrm{H} 3 \mathrm{~K} 27 \mathrm{Ac}+/ \mathrm{PU} .1^{+}\right)$enhancer set

ð STAT6 cistrome

ð RXR cistrome

ð RXR/STAT6 co-peaks
$24 \mathrm{~h}$ attachment on culture dish
Cell treatments

IL -4
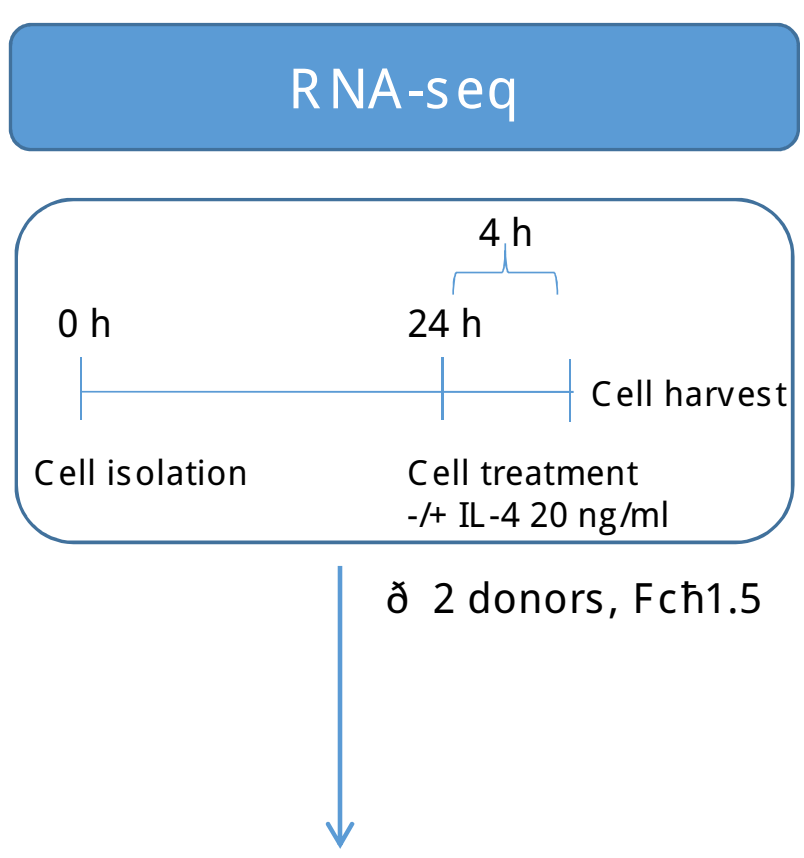
ðL-4-responsive macrophage
transcriptome 
A

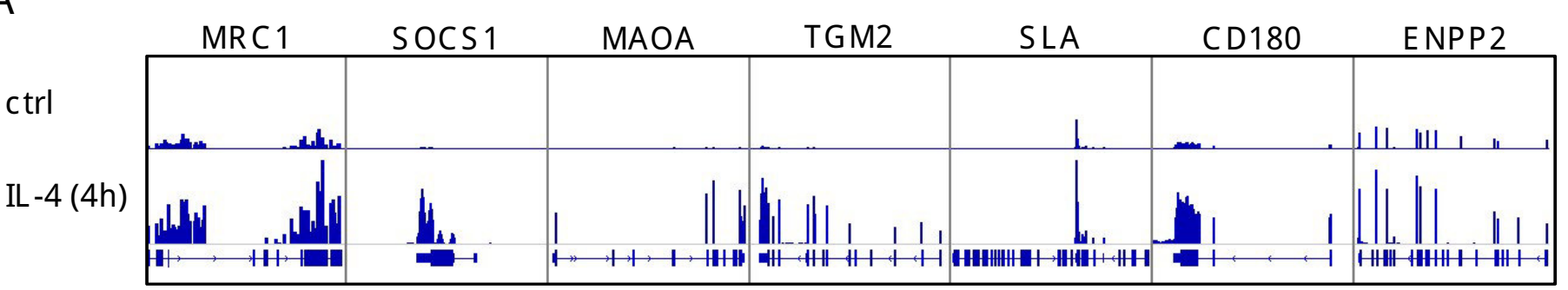

B

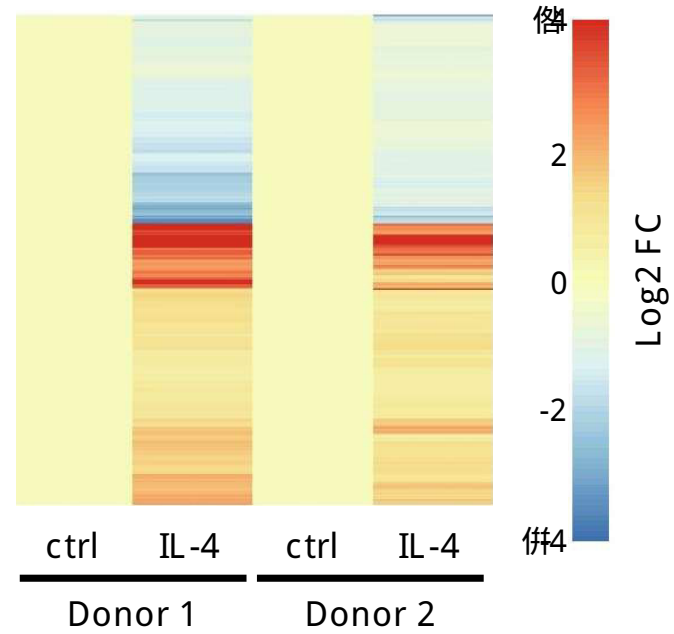

D

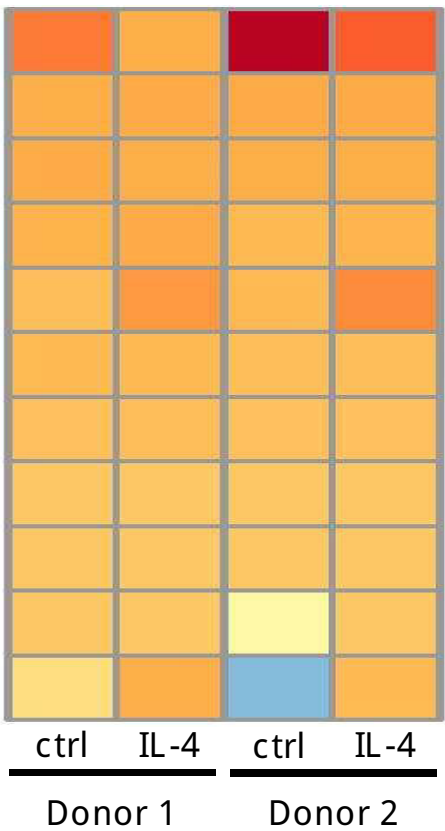

LXR

$\operatorname{LXR} \oint$

$\mathrm{RXR}$

PPAR 200

VDR

RXR $\oint$

RAR 100 주준

THR

PPAR 50

RAR $\therefore$

PPAR $\therefore$

C

\begin{tabular}{l}
1 \\
$\sim$ \\
0 \\
0 \\
\hline
\end{tabular}

Biological process Gene No. p-value FDR

$\begin{array}{cccc}\begin{array}{c}\text { inflammatory response } \\ \text { positive regulation of } \\ \text { inflammatory response } \\ \text { immune response }\end{array} & 56 & 1.27 \mathrm{E}-16 & 2.00 \mathrm{E}-13 \\ \begin{array}{c}\text { cellular response to } \\ \text { interferon-gamma } \\ \text { chemotaxis }\end{array} & 16 & 6.12 \mathrm{E}-11 & 1.11 \mathrm{E}-07 \\ \begin{array}{c}\text { chemokine-mediated } \\ \text { signaling pathway }\end{array} & 16 & 3.41 \mathrm{E}-09 & 6.20 \mathrm{E}-06 \\ \begin{array}{c}\text { response to } \\ \text { lipopolysaccharide } \\ \text { signal transduction }\end{array} & 24 & 1.33 \mathrm{E}-07 & 2.41 \mathrm{E}-04 \\ \text { monocyte chemotaxis } & 12 & 9.89 \mathrm{E}-07 & 4.03 \mathrm{E}-04 \\ \begin{array}{c}\text { lymphocyte chemotaxis } \\ \text { lym }-06\end{array} & 10 & 1.01 \mathrm{E}-06 & 1.83 \mathrm{E}-03\end{array}$


A

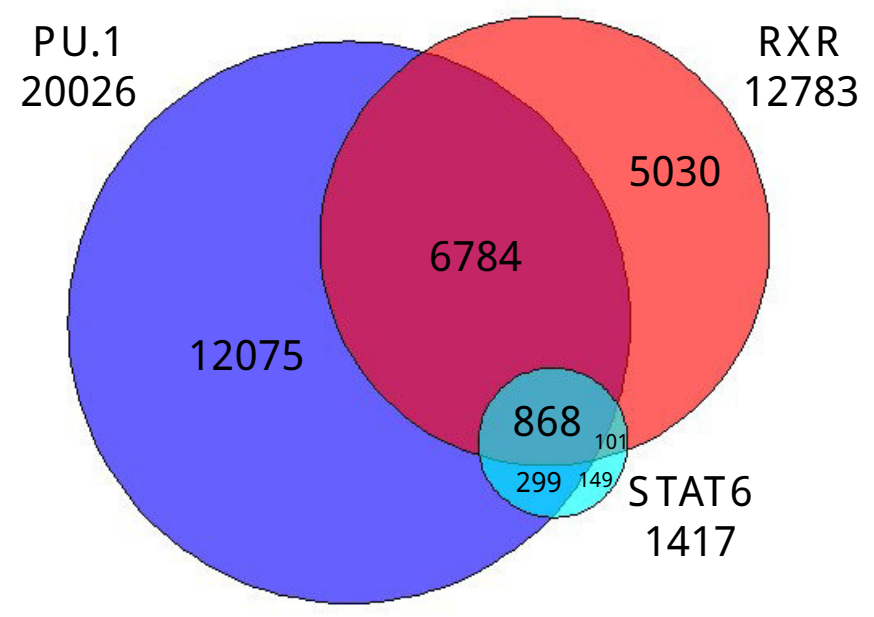

\begin{tabular}{|l|c|l|l|}
\hline Peaks (DiffBind) & PU.1 & RXR & STAT6 \\
\hline Consensus & 26531 & 14125 & 1573 \\
\hline Active (H3K27ac) & 20046 & 12799 & 1417 \\
\hline
\end{tabular}

C

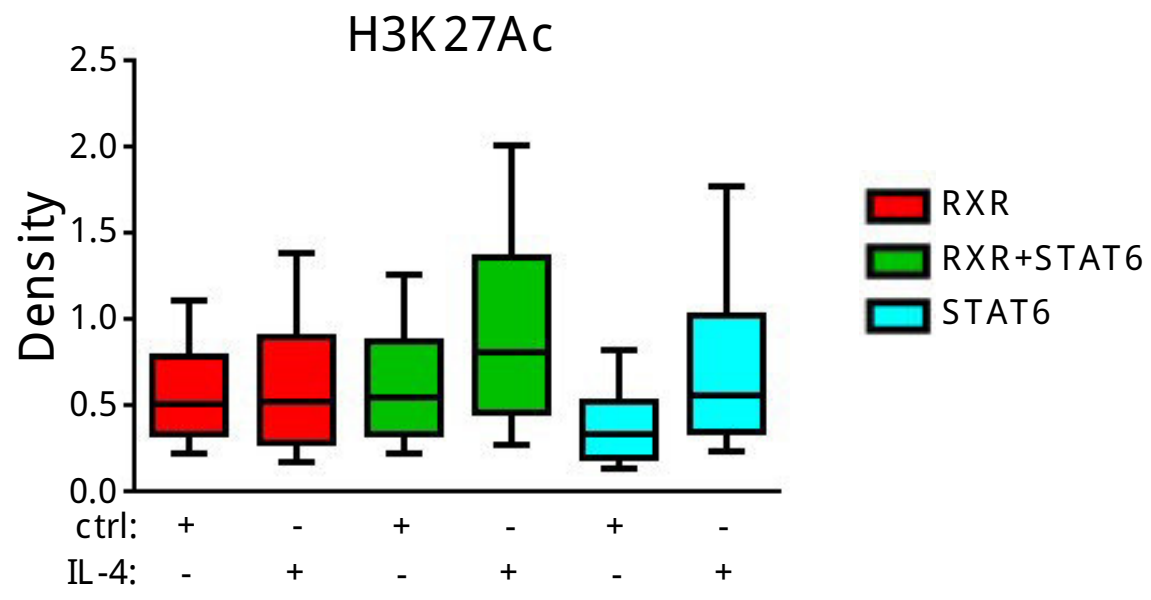

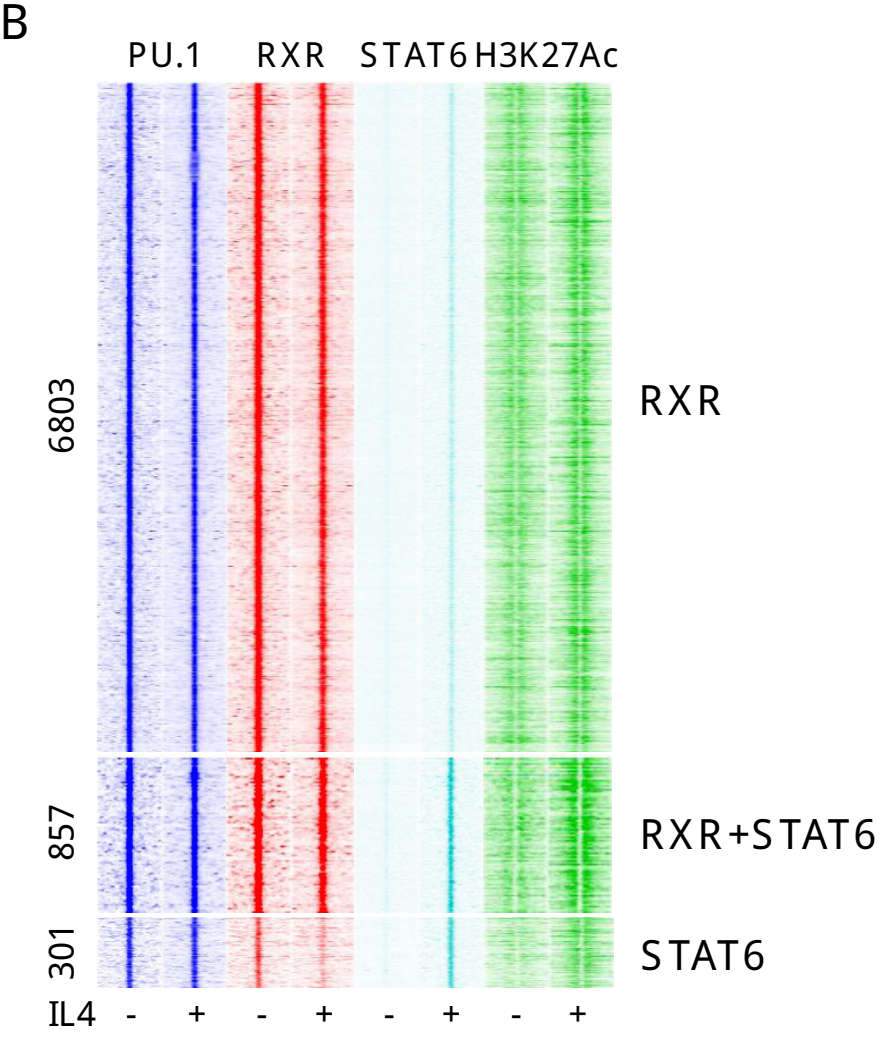


A
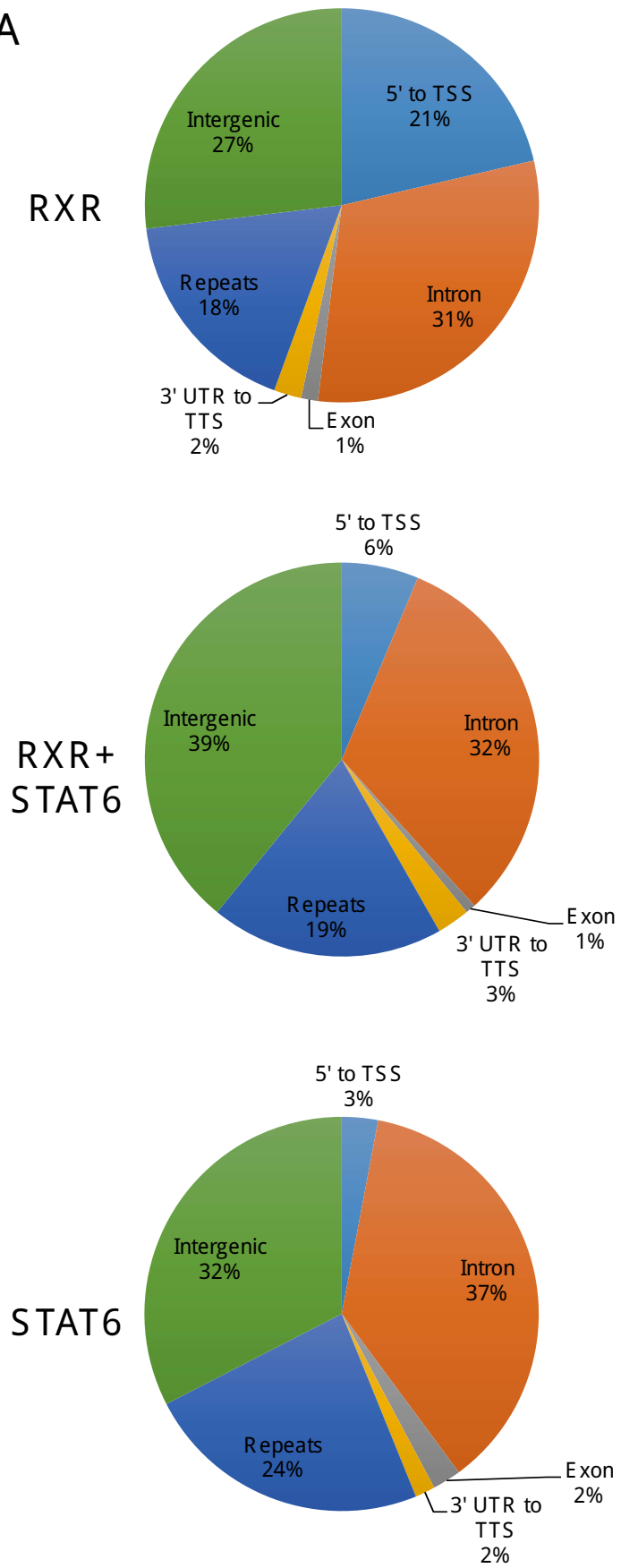

B

\begin{tabular}{|c|c|c|c|c|}
\hline \multicolumn{5}{|c|}{$\begin{array}{l}\text { PU.1 + RXR } \\
(883 / 1000)\end{array}$} \\
\hline & p-value & Target (\%) & $\mathrm{Bg}(\%)$ & Motif \\
\hline$\therefore=G G A A s x$ & $1 \mathrm{E}-278$ & 63.19 & 12.12 & PU.1 \\
\hline$T_{x} x_{x} \mathrm{CAA}$ & $1 \mathrm{E}-75$ & 16.53 & 2.31 & C/EBP \\
\hline$G G T T A C T=E G G T C A=$ & $1 \mathrm{E}-71$ & 2.94 & 0 & DR4 \\
\hline$T_{\subseteq A_{\varepsilon}} T C A$ & $1 \mathrm{E}-62$ & 20.84 & 4.78 & AP-1 \\
\hline$=\pi G A G_{C} T C A$ & $1 \mathrm{E}-41$ & 27.75 & 11.09 & NR half \\
\hline \multicolumn{5}{|c|}{$\begin{array}{c}\text { PU.1 + RXR + STAT 6 } \\
(703 / 857)\end{array}$} \\
\hline & p-value & Target (\%) & $\mathrm{Bg}(\%)$ & Motif \\
\hline$\ldots G G A A_{-T}$ & $1 \mathrm{E}-247$ & 52.54 & 6.21 & PU.1 \\
\hline $\mathrm{TT}_{\mathrm{a}}^{\mathrm{a} A}$ & $1 E-124$ & 36.76 & 6.31 & C/EBP \\
\hline TTc $=\mathrm{GAA}$ & $1 \mathrm{E}-92$ & 36.48 & 8.63 & STAT6 \\
\hline$=$ TGAeTCA & $1 E-38$ & 20.99 & 6.2 & AP-1 \\
\hline$G G G_{A \cap T T T} T C_{c}$ & $1 E-26$ & 21.27 & 8.28 & REL \\
\hline$\Rightarrow A A C C A C$ & $1 \mathrm{E}-22$ & 10.99 & 2.86 & RUNX \\
\hline \multicolumn{5}{|c|}{$\begin{array}{l}\text { PU.1 + STAT } 6 \\
(233 / 301)\end{array}$} \\
\hline & p-value & Target (\%) & $\mathrm{Bg}(\%)$ & Motif \\
\hline$G G A A_{\cong} T$ & $1 \mathrm{E}-80$ & 61.8 & 10.25 & PU.1 \\
\hline$-T=C_{z}=G A A_{-}$ & $1 \mathrm{E}-57$ & 45.06 & 6.96 & STAT6 \\
\hline${ }_{\triangle} T_{\mathrm{sT}} \mathrm{G}_{s} \mathrm{AA} I$ & $1 \mathrm{E}-22$ & 28.76 & 7.12 & $C / E B P$ \\
\hline a TGA ICA $=$ & $1 \mathrm{E}-18$ & 16.74 & 2.72 & $A P-1$ \\
\hline
\end{tabular}


A

IL -4 responsive genes-associated

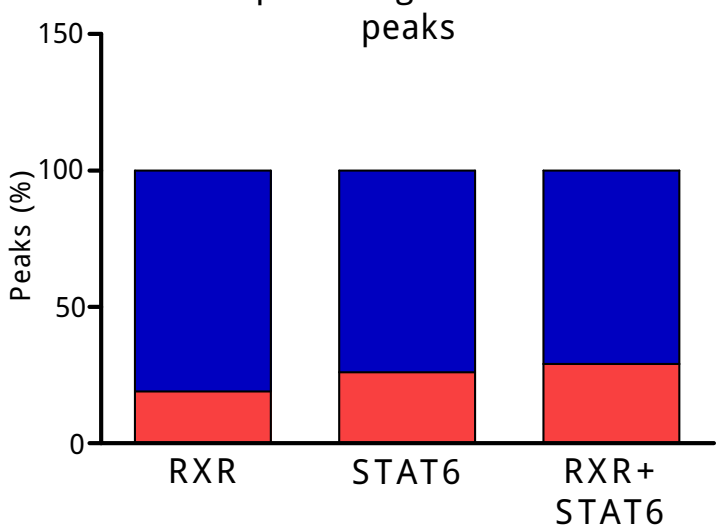

B

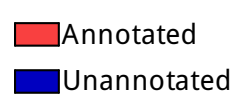

Peak distribution relative to TSS

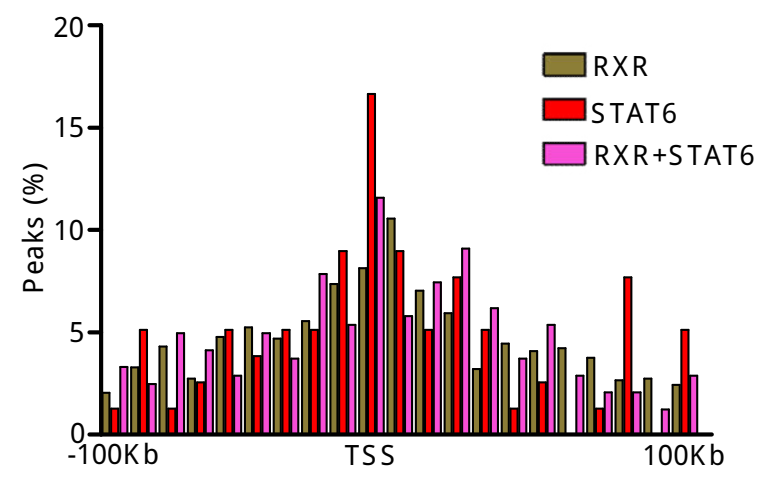

C

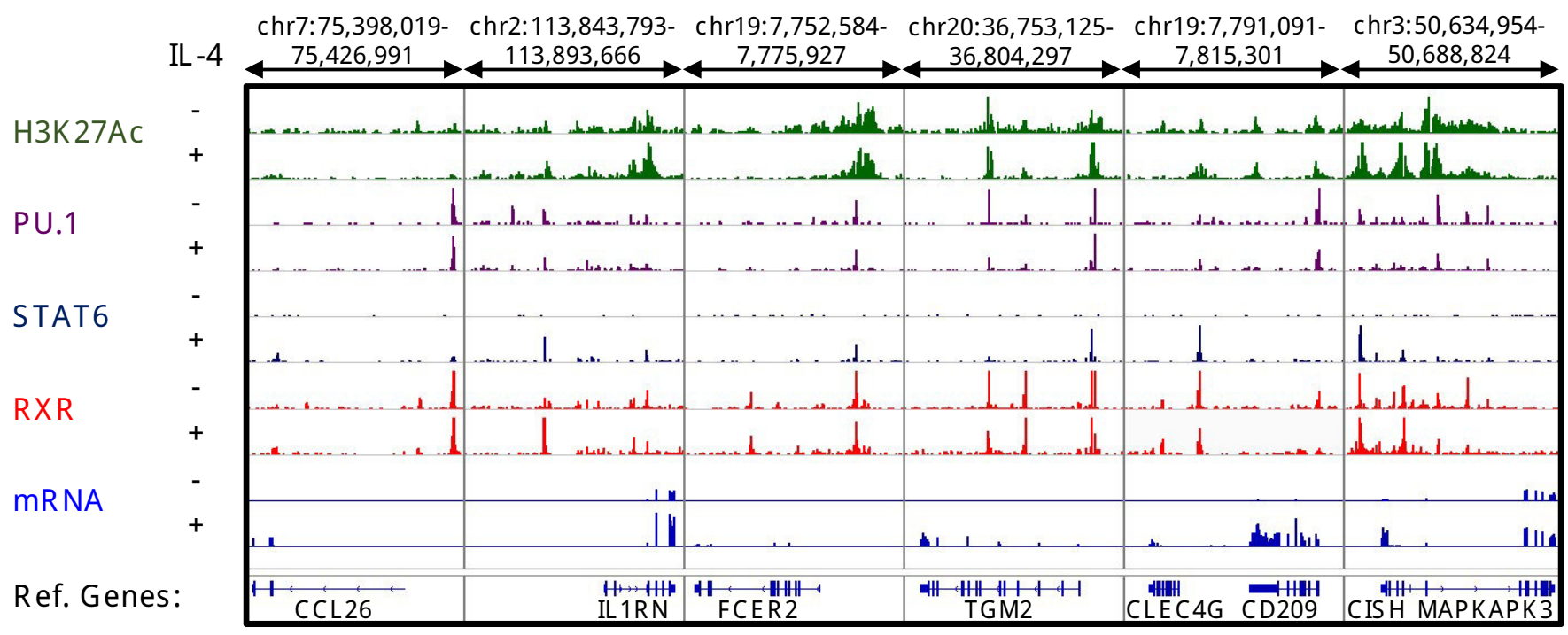


A

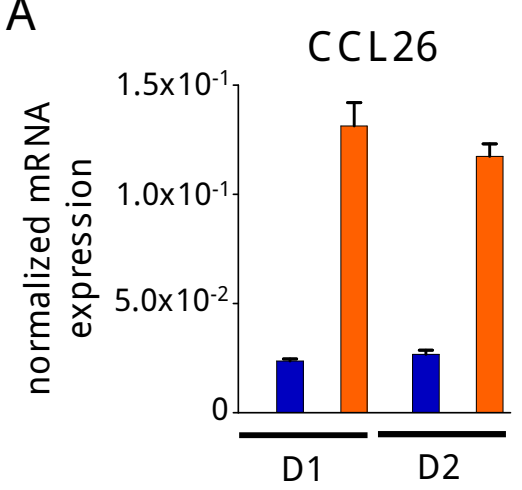

IL 1 R N

FCER2 TGM2
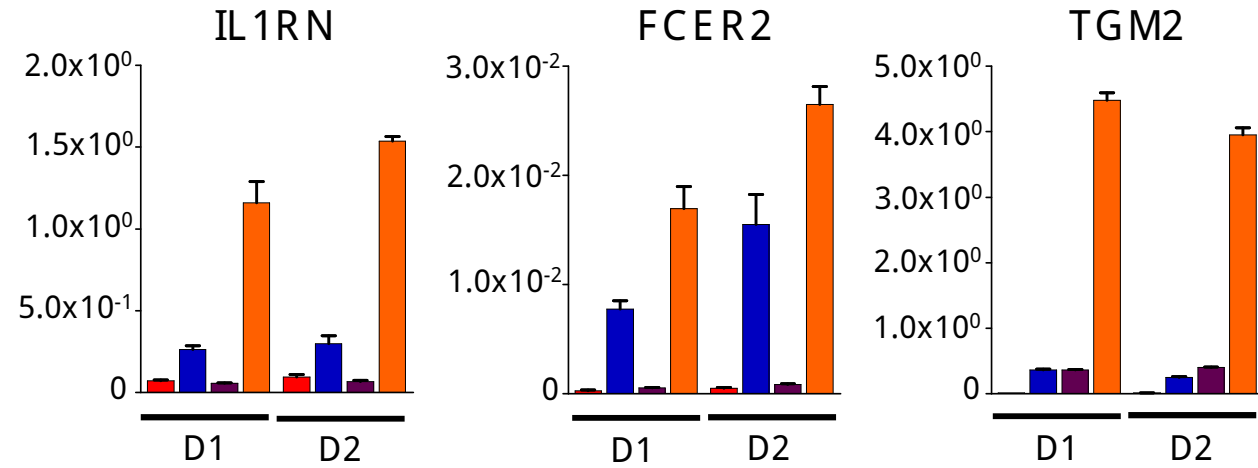

CLEC4G

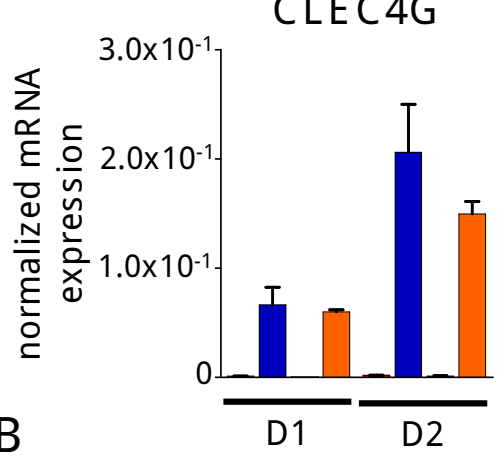

CD209
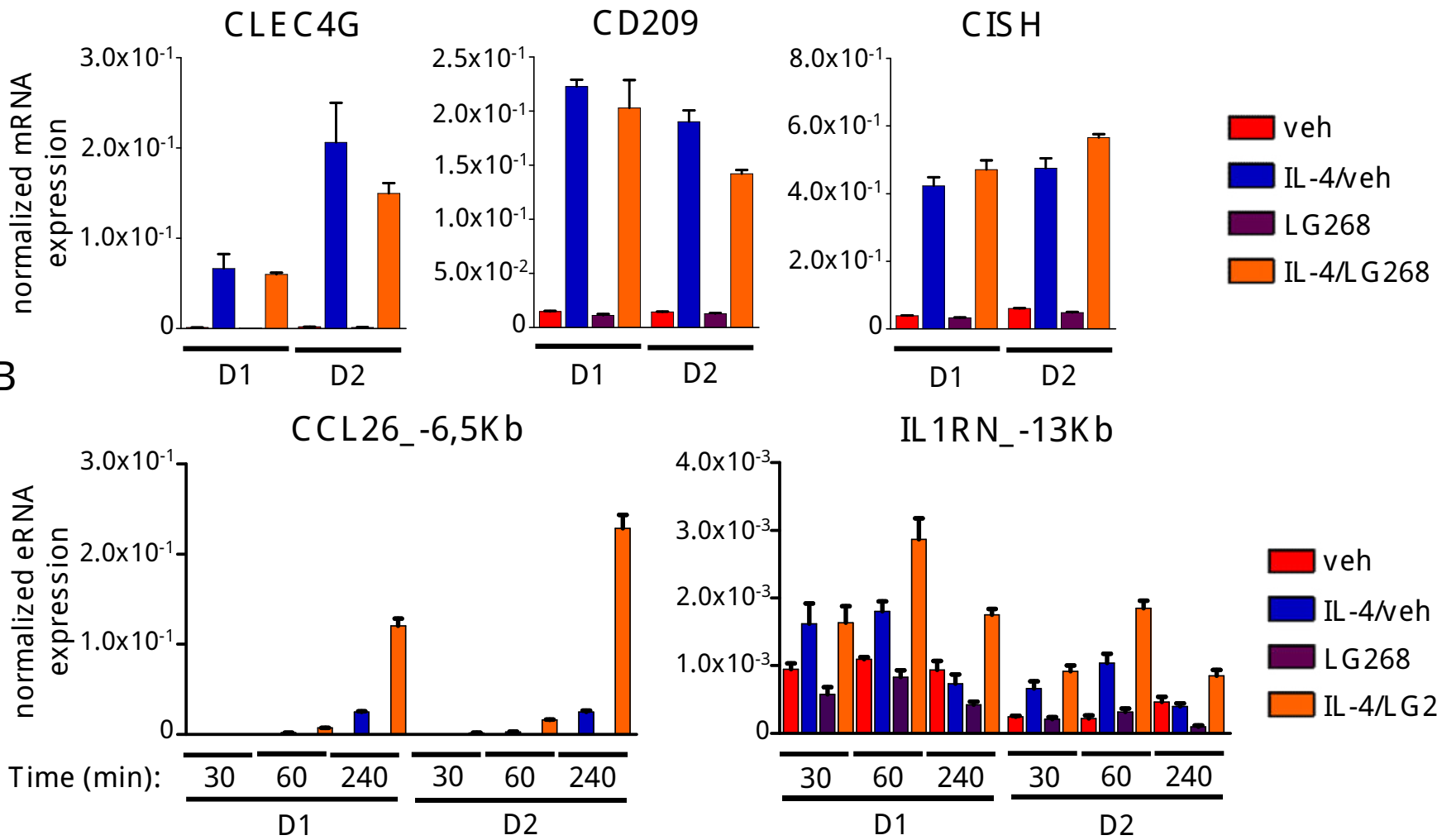

IL $1 R N_{-}-13 K b$

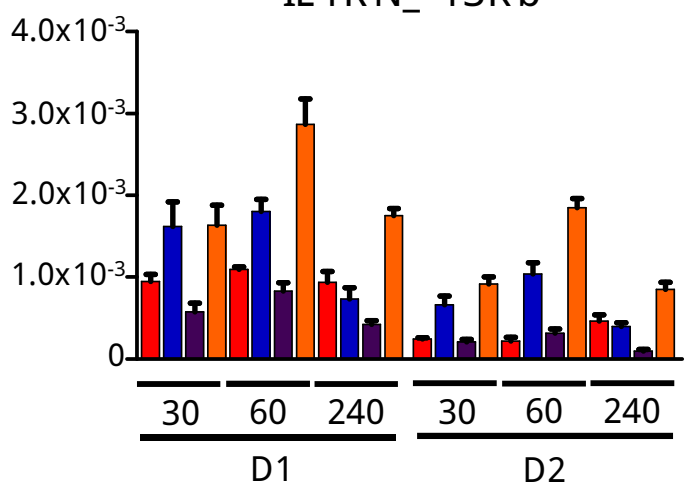

$\square$ veh

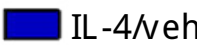

$\square$ LG268

$\square$ IL -4/L G 268

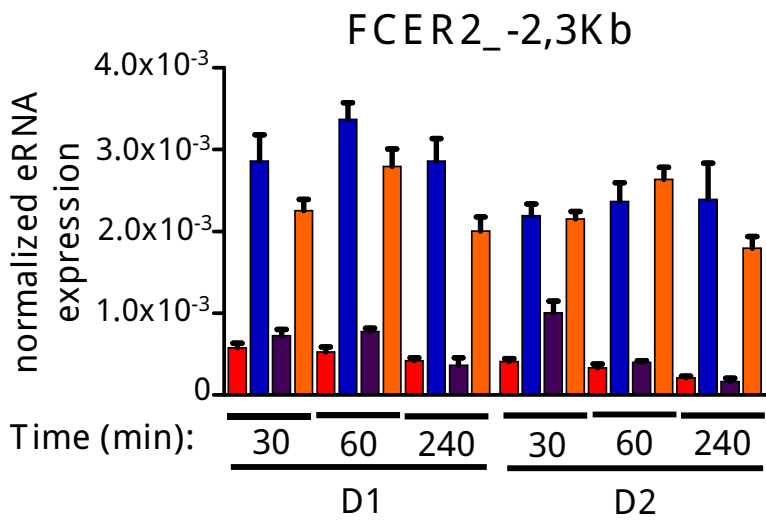

TGM2_-3,3Kb
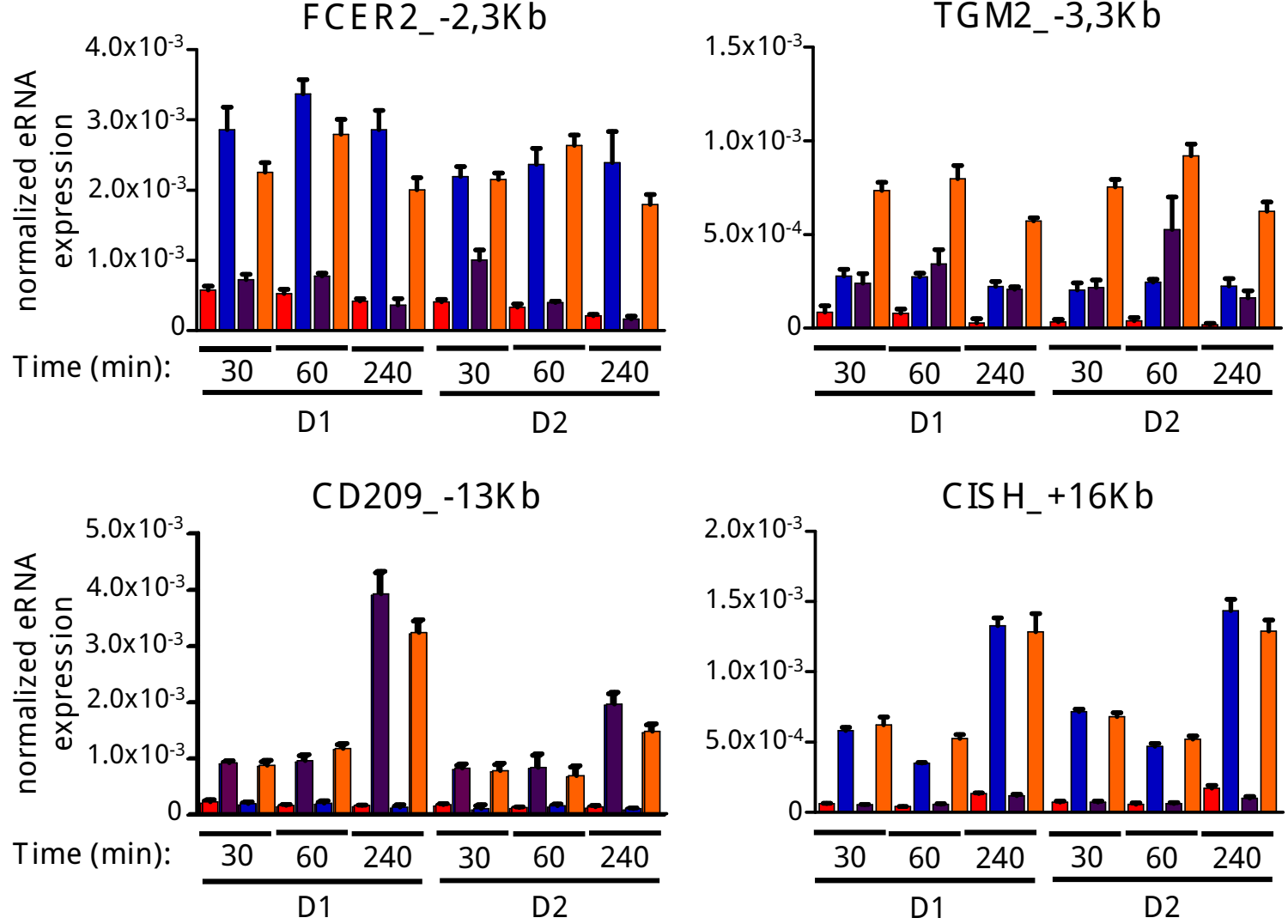
synergistic

potentiating

liganded RXR independent

veh
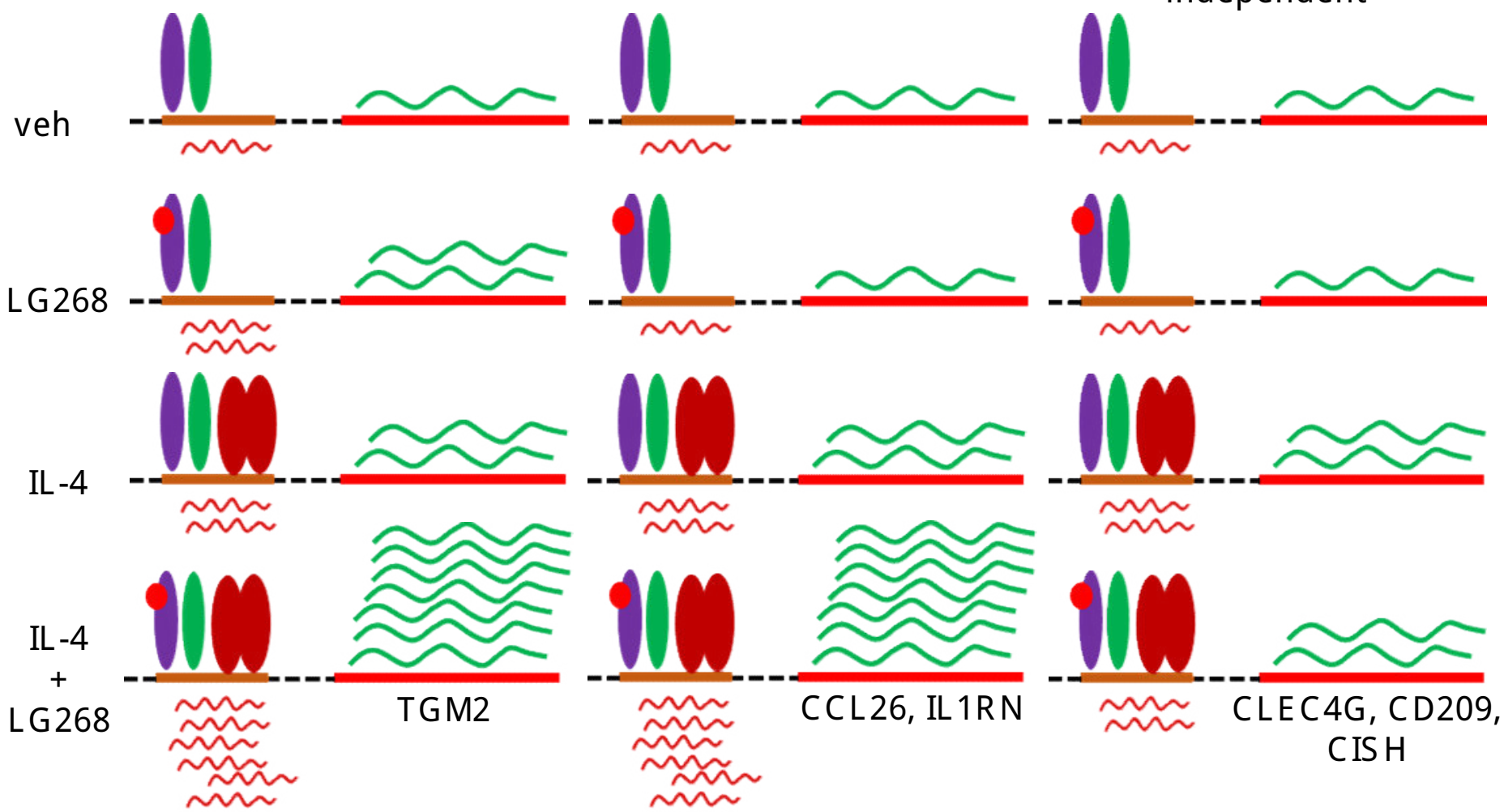

$\sim$ mRNA $\sim$ eRNA RXR NRR STAT6 LG268 